\title{
Building Operation Specialist: A Probabilistic Distributed Fault Detection, Diagnostics and Evaluation Framework for Building Systems
}

\author{
by \\ Zixiao Shi
}

A thesis submitted to the Faculty of Graduate and Postdoctoral Affairs in partial fulfillment of the requirements for the degree of

\author{
Ph.D. \\ in
}

Civil Engineering

Carleton University

Ottawa, Ontario

(C) 2018, Zixiao Shi 


\section{Abstract}

The scope of this thesis is to develop an automated fault detection, diagnostic, and evaluation (AFDDE) framework for building systems. This framework aims to provide a holistic approach to detect, identify and evaluate building faults to the stakeholders to facilitate decision-making. It is adaptable to different building systems as well as flexible to both distributed and centralised implementations. The first component of the framework, fault detection, uses a novel technique called constrained dual Extended Kalman Filter (EKF) to estimate system parameters and then generates symptom descriptions described by probability and severity. The fault diagnostic process uses Dynamic Bayesian Network (DBN) with leaky Noisy-Max model to accommodate probabilistic descriptions of faults and symptoms. The fault evaluation aspect of the system employs existing building performance simulation (BPS) tools to estimate quantitative impacts of the diagnosed faults. A model reduction process called "model-cluster-reduce" is also developed to speed up simulation. Each component of the framework is created with the intention to be generalized to other related areas of research such as model predictive control and BPS optimization. Four case studies of both zone-level and air handling unit (AHU)-level are adopted to demonstrate the functionalities of the proposed AFDDE framework. Overall, the framework shows promising results with a short fault diagnosis time, and low false positive and false negative rates, albeit with the tendency of overestimating fault impacts. In addition to the future work to further expand the AFDDE framework, many fundamental research questions also arise from this thesis. 


\section{Acknowledgements}

First and foremost, I would like to thank my Ph.D. supervisor, Dr. Liam O'Brien for his guidance and supervision throughout my study. It was a great pleasure to work with him, and I would not have accomplished this work without his mentoring and encouragement. He has aspired me to continue my academic career and pursue my research in building science.

I would also like to express my appreciation to Dr. Burak H. Gunay, Jayson Bursill, Dr. Sara Gilani, Dr. Aly Abdelalim and Dr. Scott Bucking. Your knowledge helped me through many challenges during my study, our discussions inspired me countless times, and our collaborations have been impactful to me.

I thank the encouragement and guidance I received from the colleagues I met through ASHRAE and IBPSA, fellow researchers from Autodesk Research and Canada National Research Council, as well as industry experts from Rycom inc. Your multi-disciplinary expertise helped me expand my vision. Thank you to Autodesk, NSERC, and ASHRAE for financially supporting my research.

Finally, I want to thank my wife, Yitian $\mathrm{Hu}$, for her encouragement and support during my Ph.D. study. My parents, Dengrong Shi and Jinlian Yu, for their influence and advice throughout all these years. 


\section{Table of Contents}

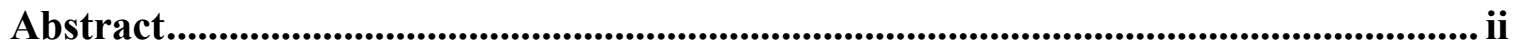

Acknowledgements ......................................................................................................ii

Table of Contents ............................................................................................................ iv

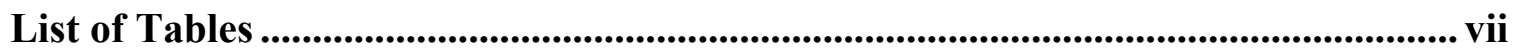

List of Illustrations................................................................................................................... viii

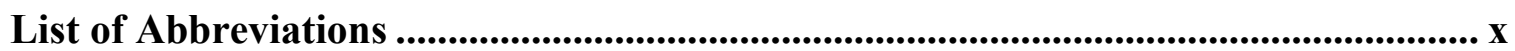

Nomenclature (....................................................................................................................... xi

1 Chapter: Introduction ....................................................................................................... 1

$1.1 \quad$ History of FDD in Buildings ………………………

1.2 An Outlook of AFDD in Buildings …………………………………………….... 6

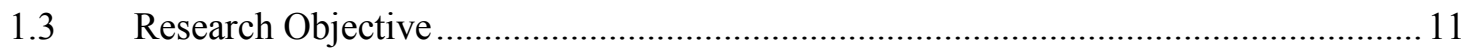

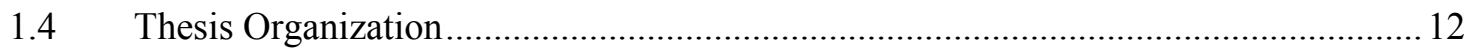

2 Chapter: Literature Review.......................................................................... 14

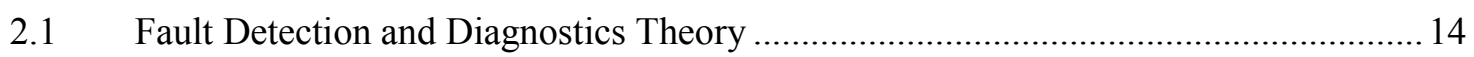

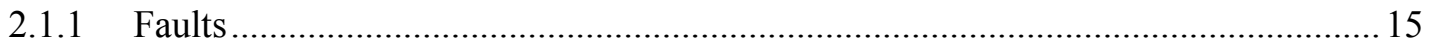

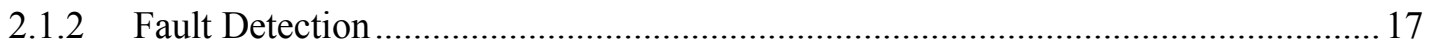

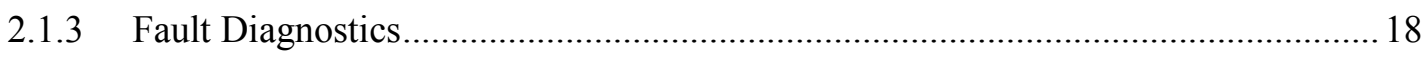

2.2 Overview of AFDD Research in Building Systems ..................................................2 20

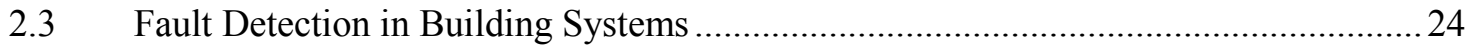

2.3.1 Limit checking and trend checking ..................................................................2

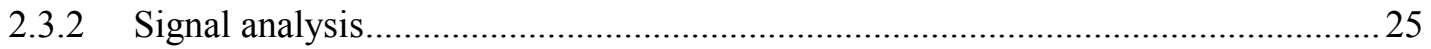

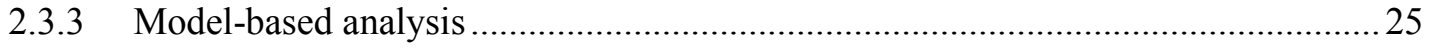

2.3.3.1 Qualitative models......................................................................................2

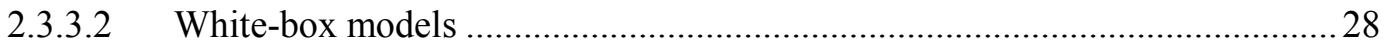




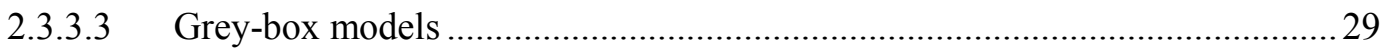

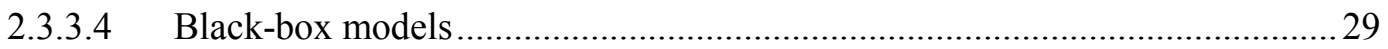

2.3.4 Review of fault detection methods in building systems....................................... 30

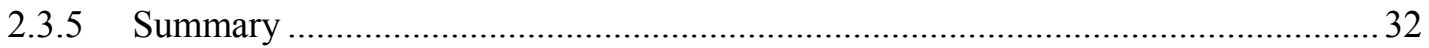

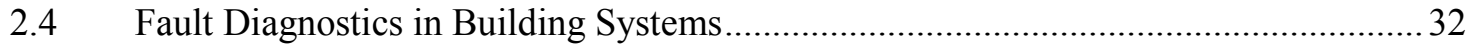

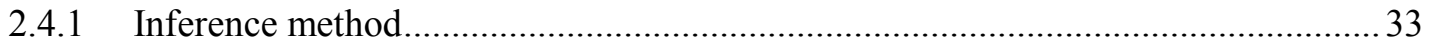

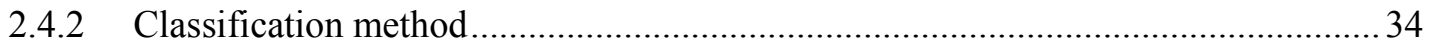

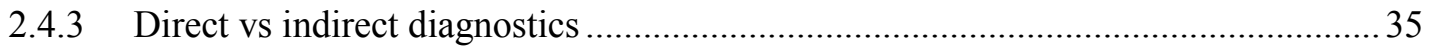

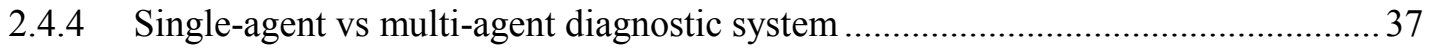

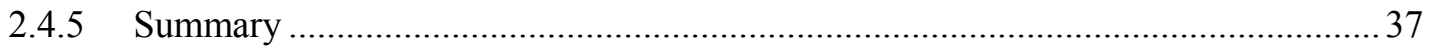

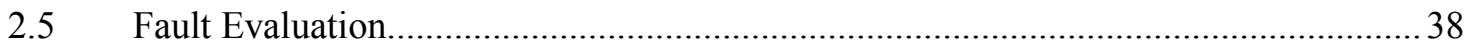

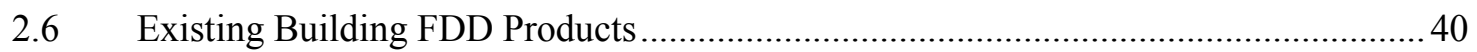

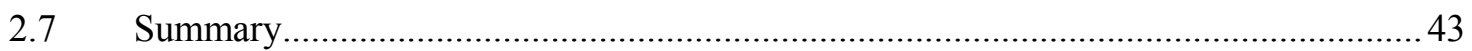

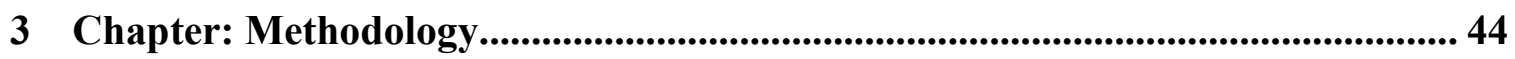

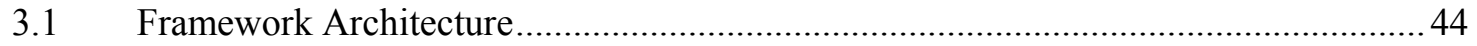

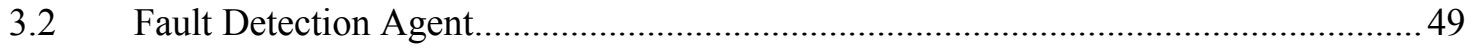

3.2.1 Parameter estimation using constrained dual Extended Kalman Filter ....................49

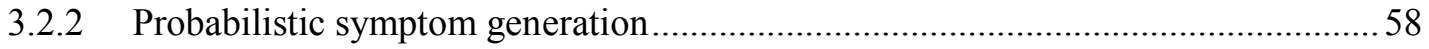

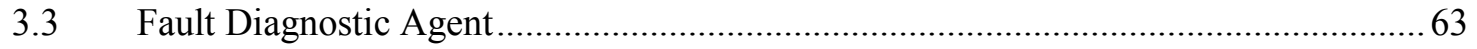

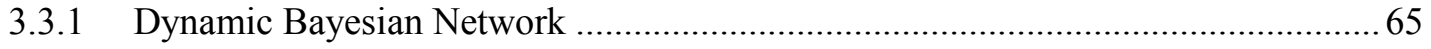

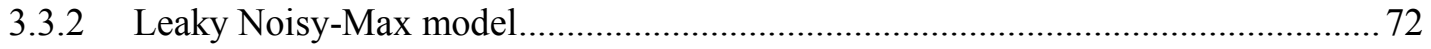

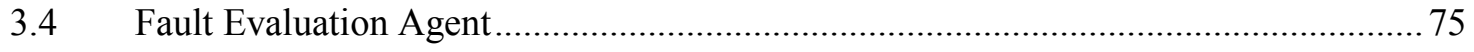

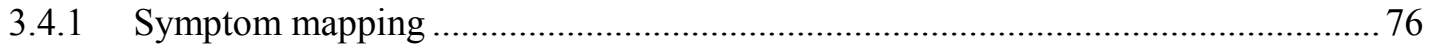

3.4.2 Multi-level simulation using model reduction ..................................................... 78

3.4.3 Model-Cluster-Reduce Method for Model Reduction .......................................... 80

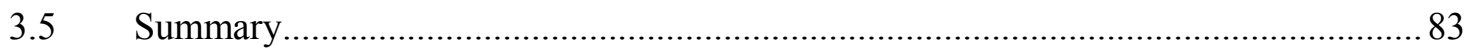


4 Chapter: Results...................................................................................................... 85

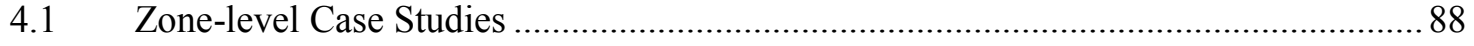

4.1.1 Shoebox Model Simulation.......................................................................... 91

4.1.2 Single Zone: Experiment................................................................................96

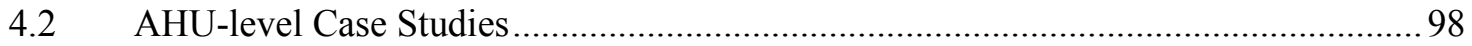

4.2.1 ASHRAE RP-1312 ............................................................................99

4.2.2 Simulation-based Case Study ............................................................................. 103

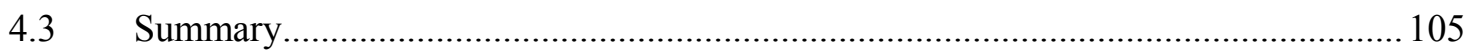

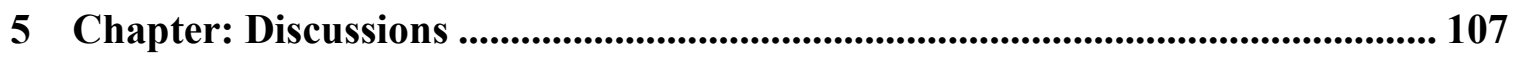

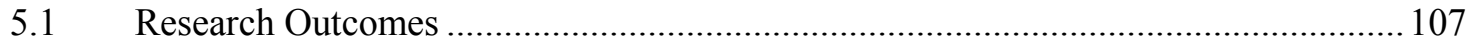

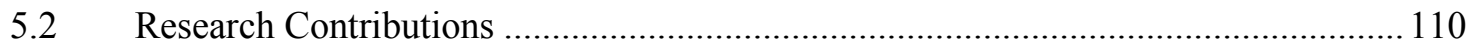

$5.3 \quad$ Future Work

6 Chapter: Conclusions .................................................................................................. 117

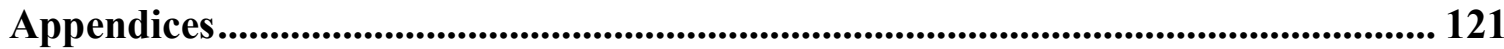

Appendix A Table of reviewed articles ........................................................................... 121

Appendix B R Code for Dynamic Bayesian Network Fault Diagnostics................................. 126

Appendix C Python Code for Constrained Dual EKF........................................................... 129

Appendix D Python Code for Model-Cluster-Reduce............................................................. 134

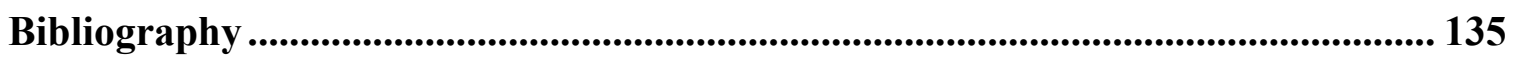




\section{List of Tables}

Table 1-1 Comparison of different FDD approaches 10

Table 2-1 Comparison of symptoms generated from fault detection process $\quad 18$

Table 2-2 Comparison of approaches to automated fault diagnosis 20

Table 2-3 Comparison of different model-based fault detection methods 27

Table 2-4 Comparison of fault impact evaluation approaches $\quad 40$

Table 2-5 Survey of existing FDD products 42

Table 3-1 Overall algorithm of Constrained dual filter 52

Table 3-2 Examples of symptom description $\quad 59$

Table 3-3 Templates for logistic regression 62

Table 3-4 Example of typical faults in building systems $\quad 77$

Table 3-5 Examples of symptom mappings to EnergyPlus 78

$\begin{array}{ll}\text { Table 4-1 Overview of the case study results } & 87\end{array}$

Table 4-2 List of symptoms generated inside the zone fault detection agent 90

Table 4-3 Faults implemented at the zone level FDDE system 90

Table 4-4 Fault diagnostics results for simulation-based zone-level case study 94

Table 4-5 Fault Evaluation results for simulation-based zone-level case study 95

Table 4-6 Summary of the FDD results from the measured zone-level case study 98

Table 4-7 Symptoms created for AHU-level implementation 99

Table 4-8 AHU-level fault-symptom relationships 99

Table 4-9 Fault diagnostics results for measurement-based AHU-level case study 101

Table 4-10 FDD results for the AHU-level simulation case study 105

Table 4-11 Fault evaluation results for the AHU-level simulation case study 105 


\section{List of Illustrations}

Figure 2-1 Sample technical process with faults ........................................................ 16

Figure 2-2 Example of fault-symptom relationship [28], left: from fault to symptom

inside a physical system; right: from symptom to fault inside a diagnosis system .......... 19

Figure 2-3 Number of articles per year surveyed in this literature review ....................... 23

Figure 2-4 Survey of different fault detection methods................................................... 31

Figure 2-5 Comparison of different fault detection methods before and after 2010 ........ 32

Figure 2-6 Comparison of direct and indirect diagnostics approach by year ................... 36

Figure 3-1 Overview of the distributed fault detection, diagnostics and evaluation

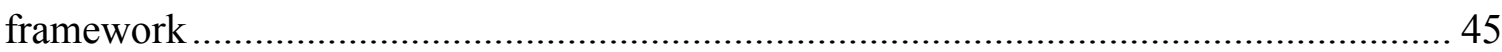

Figure 3-2 Symptom, fault and impact used in the framework ....................................... 46

Figure 3-3 Overview of dual estimation with EKF/UKF. Each estimator can be tweaked

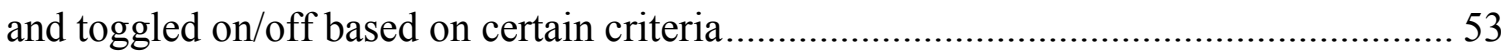

Figure 3-4 Example of a diverged parameter causing the reduced model to become

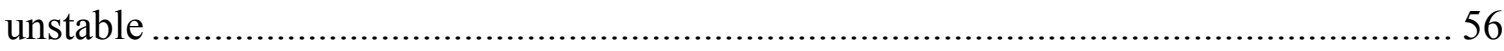

Figure 3-5 Sensitivity analysis for parameter estimation. From a) to d): wall thermal resistance, zone infiltration, equipment loads during occupied hours, constant equipment load. One standard deviation is used as the error bar. 58

Figure 3-6 Example of different logistic regression curves for calculating ps: $\mathrm{z} 0$ is the position of $\mathrm{z}$ score where symptom starts occurring, $\mathrm{k}$ is the slope of the logistic

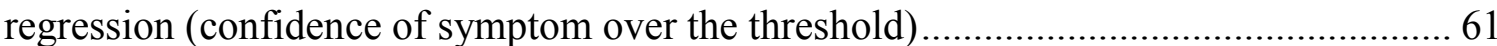


Figure 3-7 Example of a symptom probability calculated from different logistic regression templates. Above: calculated from the Medium template; Below: calculated

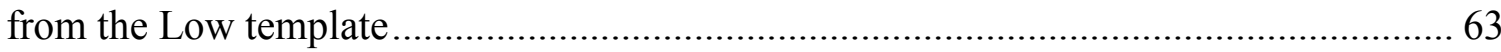

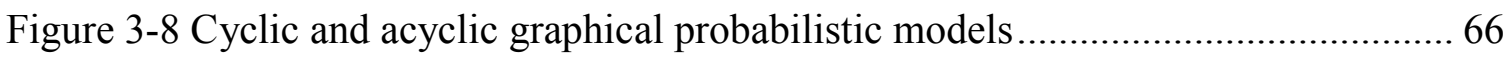
Figure 3-9 Example of a BN used for VAV level fault diagnostic, the arrows denote causal relationships between the node and starts from cause to effect; shaded nodes are faults. 68

Figure 3-10 Example of fault propagation from plant level to VAV level. 69 Figure 3-11 Example of the Dynamic Bayesian Network, fault probabilities from a previous time step affect the fault probabilities of the next time step 70 Figure 3-12 Example of fault probabilities of using BN and DBN (published in ASHRAE

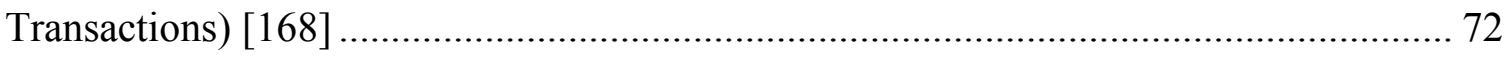

Figure 3-13 Schematics of the Model-Cluster-Reduce Pipeline [178] .......................... 81 Figure 4-1 Example of the metrics used to evaluate the FDDE framework .................... 86 Figure 4-2 Reduced model used for zone parameter estimation................................. 89

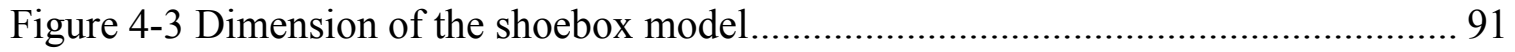
Figure 4-4 Example of the FDD results for simulated zone study; the faults start at 100 time step 94

Figure 4-5 Image of an unintended window opening fault causing freezing pipes and water damage . 97

Figure 4-6 overview of the building models. Left: BIM model; right: EnergyPlus model 103 


\section{List of Abbreviations}

\begin{tabular}{|c|c|}
\hline$A F D D$ & Automated fault detection and diagnostics \\
\hline$A F D D E$ & Automated Fault fault detection, diagnostics and evaluation \\
\hline$A H U$ & Air handling unit \\
\hline$B A S$ & Building automation system \\
\hline$B E M$ & Building energy management system \\
\hline$B I M$ & Building information modelling \\
\hline$B N$ & Bayesian Network \\
\hline$B P S$ & Building performance simulation \\
\hline$C P T$ & Conditional probability table \\
\hline$D B N$ & Dynamic Bayesian Network \\
\hline$E K F$ & Extended Kalman filter \\
\hline$E U I$ & Energy usage intensity \\
\hline$G H G$ & Greenhouse gas \\
\hline$H V A C$ & Heating, ventilation and air conditioning \\
\hline$H V A C \& R$ & Heating, ventilation, air conditioning and refrigeration \\
\hline$M P C$ & Model predictive control \\
\hline$P P D$ & Percentage dissatisfied \\
\hline$U K F$ & Unscented Kalman filter \\
\hline$V A V$ & Variable air volume \\
\hline
\end{tabular}




\section{Nomenclature}

\begin{tabular}{|c|c|}
\hline$\lambda$ & Forget factor \\
\hline$\mu$ & Scale factor for model reduction \\
\hline$\Phi$ & Parameters of a technical process \\
\hline$c$ & Leak and compound probabilities \\
\hline$F$ & Fault \\
\hline$f$ & Fault state \\
\hline$I(S, F)$ & Influence from fault to symptom \\
\hline$K$ & Kalman gain \\
\hline$N$ & Noise \\
\hline$N(\mu, \sigma)$ & Gaussian noise with $\mu$ mean and $\sigma$ covariance \\
\hline$P$ & Covariance matrix for state and parameter vector \\
\hline$P(F)$ & Prior probability of fault \\
\hline$P(S \mid F)$ & Conditional probability of symptom based on fault \\
\hline$R$ & Noise covariance for model and observation \\
\hline$S$ & Symptom \\
\hline$s$ & Symptom state \\
\hline$t$ & Time step $\mathrm{t}$ \\
\hline$x$ & States of a technical process \\
\hline$Y$ & Process Output \\
\hline$Z$ & Symptom severity \\
\hline
\end{tabular}




\section{Chapter: Introduction}

The inception of modern heating, ventilation and air conditioning (HVAC) systems and electrical lighting systems around the turn of the $20^{\text {th }}$ century has dramatically improved indoor environment inside buildings. The general population, especially in developed countries, has become used to liberally using energy sources such as electricity and natural gas to maintain persistent comfortable indoor conditions. In turn, energy usage in buildings has contributed significantly to the explosive growth of the world's energy consumption since the mid- $20^{\text {th }}$ century [1]. Increased burning of fossil fuel for electricity generation and heating lead to humanmade climate change and energy crisis [1]. By 2016, buildings account for $20 \%$ of world's energy consumption and greenhouse gas (GHG) emissions [2], more than half of which is used for maintaining indoor environment through heating, cooling, ventilation, and lighting. As a collateral effort to curtain carbon emissions and reduce the speed of global warming, most countries have pledged to reduce their energy use and GHG emissions in the recent Paris Agreement [3]. Buildings, as one of the primary energy consumers and carbon emitters, have the most potential to achieve "significant and cost-effective GHG emissions reductions" according to the United Nation [4]. As a result, many "green building" initiatives have been developed over the past decades such as LEED [5] and Passive House [6] to guide sustainable building designs and operations.

Energy sources may become more sustainable in the future, yet keeping building systems operating under optimal conditions will always be an important research topic. Given the limited natural resources and their unequal distributions, it seems inevitable that there will be inequality. As of now, 25\% of world's population with USD $\$ 10,000$ GPD per capita or more consumes $60 \%$ of total energy usage [7], while $30 \%$ of world's population still lives in slum-like conditions 
according to UN-Habitat [8]. Furthermore, the World Health Organization estimated that around two million people die each year from poor indoor quality caused by burning biomass or coal indoors with inappropriate stoves [9]. Increasing buildings' operation efficiency could significantly improve the affordability of a comfortable indoor environment, thus decreasing inequality on a larger scale.

Thankfully, recent technological advancements, especially cost-effective semiconductors, has made many advanced building technologies more affordable, such as light emitting diodes (LED), wireless sensors, and more complicated machinery such as variable flow compressors. The combination of these high-tech products and the requirement for more sustainable buildings has created a new type of the so-called high-performance buildings. Modern high-performance buildings are designed to maintain an optimum indoor environment, while at the same time minimizing energy and environmental impacts. However, complex systems are complicated to supervise and maintain. This system complexity means many high-performance buildings may lack the robustness to maintain proper performance. For instance, $25 \%$ of the LEED-rated buildings have "significantly worse" energy performance than their design target [10]. This inefficiency in energy use can be improved by reducing and correcting operational problems (faults). By optimizing building operation, buildings can achieve an energy consumption reduction up to $20 \%$ to $30 \%$ [11].

The study to reduce the probability of unintended faults and maintain systems' performance within desired conditions forms the sub-discipline of reliability engineering. Historically reliability research on building systems has mainly been focused on safety, structure reliability 
and equipment reliability. On a broader perspective, reliability engineering in buildings contains a wide range of topics, such as:

- Improving building systems' robustness through better design and manufacturing. Examples include earthquake-resistant structural design and backup boilers for hot water systems. This approach often focuses on avoiding safety hazards and total system failures on the whole building scale.

- Improving fault tolerance of a system. When a fault occurs, a fault tolerant system can maintain its performance for a specified period, giving time for repair, maintenance or evacuation. This is usually critical for safety-related issues, and is commonly applied in fire safety and earthquake practices - buildings are designed to tolerate those events to make time for evacuation. For fault tolerance application related to system performance, examples include a reheat coil that is tolerant to inappropriate supply fluid pressure without leaking, or an air handling unit (AHU) tolerant to incorrect human inputs.

- Implementing fault prognostics and preventative maintenance. One way to avoid fault is to predict them before occurring; this is called prognostics. Common prognostics include periodical equipment checking and maintenance, or replacing old parts that may fail soon. Combined with fault-tolerant controls and robust designs, these efforts can reduce the likelihood of a fault occurring in the first place, thus improve operational efficiency and reduce costs.

- Enhancing fault readiness. This approach usually involves establishing a safety and maintenance plan to efficiently solve problems caused by unexpected faults. Modern building management practices typically have very effective strategies to deal with 
faults. However, if other aspects of building reliability are improved, it can help reduce capital costs to deal with these issues reactively.

- Actively identifying and diagnosing faults. Issues in the building are usually masked by the complexity of their systems. To correct such issues, root causes of the faults need to be identified and diagnosed. Automated or manual fault detection and diagnostics (FDD) processes are used to achieve this. In conventional building operation, this task is often driven by complaints, and manual FDD deviates operators' time to perform normal operations. Modern automated fault detection and diagnostics (AFDD) aims to achieve this in a fast and accurate manner and to present suspected faults to the operators for further analysis. There have been several types of research on self-correcting building systems but is still rare compared to using AFDD as a decision aid for building operators.

Performance-related faults arerelatively common inside buildings. One survey conducted by Building Research Establishment indicated reported that 19\% -64\% of different types of buildings has defects or faults [12]; another survey in California based on 13,000 air conditioning systems indicated $65 \%$ residential units need repair and $71 \%$ of commercial units need repair [13]. The recent development of more complex building systems and autonomous control systems have led to the rise in the AFDD research in building engineering systems. As of now, most work in building FDD research and industry focus on performance-related issues such as heating, ventilation, air conditioning and refrigeration systems (HVAC\&R) [14][15]. While most of the fault prognostics research in the building industry is focused on critical issues such as building structure and fire safety. Numerous research has concluded improving operation efficiency and correcting faults could significantly decrease energy waste 
and improve indoor environment quality [14], [16], [17]. This Ph.D. work tries to develop a novel fault detection, diagnostics and evaluation framework to provide more useful information delivery, thus creating better decision support for the building operators, and provide reliable guidance for timely maintenance tasks.

\subsection{History of FDD in Buildings}

Ever since human started to construct buildings as shelters and storages, we have come up with methods to monitor buildings' health and to diagnose faults. For instance, before we had chemical or electronic carbon monoxide detectors, we used birds to detect such dangerous built environment, thus the term "canary in a coal mine". Codes of constructing, monitoring and maintaining buildings have been developed as early as 2000BC [18]. In many ancient cities, watchtowers are manned to detect early building fire and provide a fast response. Throughout history, a wide range of proactive and reactive approaches have been adopted for keeping built environments operating under desired conditions.

After the introduction of modern HVAC systems, envelope systems and electrical systems building have become ever so complicated. The importance of early fault detection has been noted since then, such as a newspaper article in 1952 emphasizing the importance of early maintenance to avoid property damage [19]. Studies on AFDD for dynamic systems began in the 1970s [20]. However, research on AFDD in building systems did not start until the 1980s due to the cost of computational resources. Some of the early work includes detecting failures on heat pumps [21] and an air handling unit fault diagnosis system using Extended Kalman Filter [22], some of these underlying methods are still widely adopted for new AFDD research today. More research started to take flight in the 1990s, then skyrocketed after the turn of the millennium. 
One of the earliest international collaboration on building system FDD was the IEA Annex 25 during the early 1990s. It was followed by several research projects funded by ASHRAE since the mid-1990s, including RP-1020 [23], RP-1615 [24], whose datasets are still being used today.

\subsection{An Outlook of AFDD in Buildings}

AFDD is the study of using computers and operation data to automatically detect, diagnose and report faults. It is largely conducted by integrating artificial intelligence into different engineering systems. Conventionally we implement computer logic that imitates human thinking to achieve diagnoses of faults. In recent years the development of statistical learning, or machine learning, has changed our perspective on how to approach this problem. Instead, it is possible to let the computer learn automatically from process history data. This section provides an overlook of how this artificial intelligence revolution means for building AFDD research and future challenges of its implementation. The details of specific technologies are further reviewed in Chapter 2.

Reasons for the rise of the AFDD market and in general, automated building operation and management from the industry can be contributed to the following factors:

1. Competitive commercial real-estate market. The recent development of space sharing, telecommuting as well as electronic commerce has led to a need for affordable commercial space. To remain competitive, commercial real estate providers need to provide high-quality indoor environment while keeping the operating costs low.

2. Most of the commercial buildings are ageing and requires large-scale retrofits.

3. Many of the building operators will retire in the next decade, making this time a unique opportunity to adopt more automated building operation strategies. 
4. Given the background of climate change and limited energy sources, there is an increasing emphasis on improving the energy performance of buildings. AFDD could help to keep building systems operating under the optimal condition to ensure efficiency and safety.

5. Recent development of advanced sensing technology and internet of things (IoT) has enabled the underlying infrastructure for more advanced AFDD applications.

Before delving into various approaches to building AFDD problem, several main challenges facing the development of modern AFDD systems are identified. First is the variation of building system configurations. Unlike many successful artificial intelligence applications in robotics and competitive games such as Go, building systems have vastly different characteristics. For example, unlike a mass manufactured car or robotic arm, a central AHU can have different duct sizes, various equipment sizes, sensor locations, the presence of dehumidification or humidification controls, etc. This means AFDD systems specifically designed for a particular system may not function on another building system, even if they share the same functionalities. At the same time, a generalized FDD model cannot perform well without sufficient data to train it to adapt to different configurations. Another characteristic is the limited sensing capabilities in building systems. Building systems often involve complicated nonlinear processes, with lots of interaction between different systems and processes. For example, temperature in an air-conditioned room is a zone level thermal system, and it involves many complex physical processes to achieve thermal equilibrium, such as insolation from the sun through windows, heat loss/gain to the outdoor environment, cooling/heating from radiant elements, etc. Only a handful of sensors are usually provided to monitor all these thermal processes, such as a thermostat, outdoor air temperature sensor and 
damper position of the air terminal. This leads to difficulties in monitoring different thermal processes and identifying faults within them. Furthermore, one element within a building system may affect another system. For example, an upstream low supply air temperature fault from the AHU can affect thermal comfort in rooms downstream. Compounded with the previous challenge, this means developing a general FDD model for building systems becomes even more difficult due to high nonlinearity.

In general, building AFDD systems can be divided into the four different categories. A summary is provided in Table 1-1, and details are described below:

- Pure expert system. An expert system is a manually constructed computer program based on expert knowledge. In building FDD research, one common expert system is the rulebased fault diagnoses system. It uses expert constructed if-then-else rules to perform logic analysis on sensory inputs. Expert systems have been the primary focus of conventional building AFDD research before the 2000, and most of the commercial products are based on this approach [25]. While this approach can produce tools efficiently, the requirement of expert input means the AFDD tool need to be tailored specifically for each building system, and there is the insufficient capability for this type of system to update and learn from future operation data. Still, due to its simplicity, expert systems are usually robust, computationally efficient, and suitable for mechanical equipment without complicated processes such as fans and pumps. They struggle when dealing with more complicated building systems and scaling its implementations.

- Local learning system. This kind of system uses building-level or campus level building data to train their AFDD models. Typically, pure statistical models are used. This approach has become the primary focus of recent research [14] and shows promise for 
future AFDD tools. However, to train such systems usually requires a large dataset, often including data when faults are occurring, which could be hard to obtain. Thus, they are usually applied as pure fault detection agents, which is used to detect anomalies without diagnosing their root cause using model trained by commissioned data.

- Expert-learning hybrid system. An expert-learning hybrid method is constructed structurally from expert knowledge and can learn from operation data using machine learning techniques. The ability to diagnose faults can be constructed mainly from expert knowledge without the extensive use of data with particular faults. At the same time, it is capable of updating its accuracy with operation data. This approach provides a middle ground between local learning system and expert system, which reduces the amount of data required to train the system and the effort required for manual construction. This approach is the focus of this Ph.D. research.

- Central learning system. With the growth of internet of things (IoT) and cloud computing, it is possible to train a statistical model from an amalgamation of building data to perform complicated FDD tasks. By using recent artificial intelligence research such as deep learning, with sufficiently large data it is possible to create powerful and self-adapting intelligent agents that can perform accurate FDD tasks even on building systems with different configurations. However, due to security reasons, the fact that many buildings may not be able to upload their data remotely, lack of infrastructure that provides a vast amount of building automation data, and the high cost of such algorithms, this approach is not yet realistic for the near future. 
Table 1-1 Comparison of different FDD approaches

\begin{tabular}{|c|c|c|}
\hline FDD Approach & Advantages & Disadvantages \\
\hline Expert System & $\begin{array}{ll}\text { - } & \text { Easy to setup } \\
\text { - } & \text { Robust } \\
\text { - } & \text { Fast to compute }\end{array}$ & $\begin{array}{ll}\text { - } & \text { Not generalizable } \\
\text { - } & \text { Hard to update }\end{array}$ \\
\hline Local Learning System & $\begin{array}{l}\text { - } \quad \text { Can be generalized } \\
\text { - } \quad \text { Requires little manual setup } \\
\text { - } \quad \text { Can be updated }\end{array}$ & $\begin{array}{l}\text { - Prone to overfitting } \\
\text { - } \begin{array}{l}\text { Require extensive } \\
\text { dataset }\end{array}\end{array}$ \\
\hline $\begin{array}{c}\text { Expert-learning } \\
\text { System }\end{array}$ & $\begin{array}{l}\text { - } \quad \text { Smaller training data needed } \\
\text { - } \quad \text { Can be updated }\end{array}$ & $\begin{array}{ll} & \text { Require some manual } \\
\text { set up }\end{array}$ \\
\hline $\begin{array}{c}\text { Central Learning } \\
\text { System }\end{array}$ & $\begin{array}{l}\text { - } \begin{array}{l}\text { Can automatically adapt to } \\
\text { different systems }\end{array} \\
\text { - } \quad \text { Fast (on the cloud) }\end{array}$ & $\begin{array}{l}\text { - } \begin{array}{l}\text { Requires significant } \\
\text { data collaboration and } \\
\text { computation power }\end{array} \\
\text { - } \begin{array}{l}\text { Limited by network } \\
\text { bottleneck }\end{array}\end{array}$ \\
\hline
\end{tabular}

As a decision-making tool or a decision-making aid, the actual functionality of sorting diagnosed faults is often ignored by building AFDD developers [14], [17]. While some studies have been performed investigating how different faults can affect energy efficiency and thermal comfort of a building [26], [27], there is little work on how to provide quantitative evaluations for faults being diagnosed by an FDD system. By collecting data during the fault and utilizing building performance simulation (BPS) tools, it is possible to predict future impacts of the fault and provide meaningful quantitative metrics such as energy, cost and thermal comfort to the building operators. Even in the future, when all building operation tasks are automated, this quantitative 
evaluation process can still help to prioritize maintenance tasks and prevent future deterioration of the building system.

\subsection{Research Objective}

The work of this Ph.D. thesis is mainly focused on developing a framework focusing on performance-related fault detection, diagnostics, and evaluation in building systems. The addition of fault evaluation, or fault assessment, provides more quantitative information to the decision-making process. On a technical level, this research aims to tackle those following challenges:

- Currently, the building automation system faces two information bottlenecks: computation bottleneck and network bottleneck. A distributed design is explored to provide a potential solution to these two bottlenecks, thus increasing the expandability of the framework.

- Building systems are usually equipped with limited sensors. To extract more information real-time in a reliable manner, robust parameter estimation and state prediction techniques need to be developed and validated.

- Many uncertainties arise within the AFDD process due to measurement error, modelling error and knowledge limitations. To better quantify these uncertainties, a probabilistic representation of fault detection and fault diagnostic results can be used. Specific fault detection and fault diagnostic methods need to be selected or created to accommodate these probabilistic evidence.

- There is a lack of quantitative evaluations of faults in current building AFDD research. With the help of building performance simulation tools, it is possible to map observed 
symptoms to the actual model inputs and use it to simulate the potential fault impacts. However, to achieve this objective also means the proposed framework need to be capable of capturing effects of potential faults as part of its fault diagnosis process and then translating these effects quantitatively to the building simulation engines.

During this research, many operation issues have been discovered inside the case study building (Canal Building) at the Carleton University campus. Problems such as excessive hot water circulation during the cooling period, inappropriate heat recovery system controls have been addressed. Several novel contributions applicable to other research disciplines such as model predictive control and building model reduction have also been created during this research effort.

\subsection{Thesis Organization}

This thesis is organized into six chapters: Chapter 2 provides a detailed literature review on general FDD research, and AFDD systems specialized on buildings. Different fault detection approaches and fault diagnosis algorithms are reviewed and discussed, and selection of Automated Fault Detection, Diagnostics and Evaluation (AFDDE) methods used in this work are reasoned.

Chapter 3 articulates the architecture of AFDDE framework, formulation of each component of the AFDDE framework, and generalization of some of the techniques for other research purposes.

Chapter 4 demonstrates four case study including simulation, real data, and published research data. The case studies are used to demonstrate the capability of the proposed framework and compare to some conventional AFDD methods. 
Chapter 5 discusses the implications and limitations of the proposed AFDDE framework. Future work to improve and expand this work is also suggested. Also, fundamental research topics to help improve the building fault management research are proposed. Finally, Chapter 6 will bring closure to this thesis. 


\section{Chapter: Literature Review}

This chapter starts by introducing the fundamentals of fault detection and diagnostics, followed by its application in building systems. Existing building AFDD research consist of more than 140 articles is categorized. Methods for fault detection and fault diagnostics are reviewed separately. Besides AFDD, previous research on fault evaluation is also reviewed. The author tries to catalogue some of the existing building FDD products on the market and discuss their characteristics. Detailed guidelines and suggested approaches for this research are proposed based on the literature review. Furthermore, some of the other potential research objectives emerged from existing research will be discussed.

\subsection{Fault Detection and Diagnostics Theory}

Fault detection and diagnosis of the technical process ensure the safety, efficiency, and quality of the process. FDD is typically composed of two components: 1) Fault detection to recognize fault events, and 2) fault diagnosis to identify and locate the fault cause. Fault detection often uses statistical inference, statistical decision, artificial intelligence and soft computing. Fault diagnosis often uses causal fault-symptom relationships [28], or statistical decision such as discriminant analysis. Conventional FDD systems often use a knowledge-based approach [29][30]. The processing of measured variables by instruments requires analytical knowledge, and the evaluation of observed variables by human operators requires heuristic knowledge [31]. The utilization of automated FDD (AFDD) in technical processes initially appeared in the late $18^{\text {th }}$ century to better monitor the emerging machineries during the industrial revolution. Starting from the 1970s, fast development of integrated circuitry and computers enabled the wider adoption AFDD systems in much smaller form-factors. The development of cheaper data storage 
and cloud computing has led to a surge of statistical based, or big data related AFDD methodologies. In recent years, thanks to the development of machine learning technology, it is possible to use black-box models purely based on process history data to perform AFDD tasks, without the explicit requirement of prior knowledge [32]. Limitations and outlook of using these newly developed approaches will be further discussed in this chapter. The following section will provide a brief introduction to faults, fault detection and fault diagnostics from a theoretical perspective.

\subsubsection{Faults}

Faults in buildings have been mentioned previously, however, the term "fault" is not thoroughly defined. According to Isermann [28], a fault is "an unpermitted deviation of at least one characteristic property (feature) of the system from the acceptable, usual, standard condition". A more classical definition by Himmelblau [33] is "a departure from an acceptable range of an observed variable or a calculated parameter associated with a process" [34]. To reiterate, faults are discrete states within the system that can lead to failures and malfunctions. There are two types of fault causes: external causes $F_{e}$ are environmental influences outside the technical process, such as inadequate heating supply from the central plant and fire; internal causes $F_{i}$ are inside the technical process, such as stuck dampers in AHU and broken windows. Faults can be further categorized by their time dependency into abrupt fault (stepwise), incipient fault (driftlike) and intermittent fault [28]. An abrupt fault, as its name suggests, happens in a fleeting time and creates a sudden deviation in the technical process, examples include sudden fan shutdown. Incipient fault, or drifting fault, slowly increases in severity, such as a slowly increasing sensor bias. Intermittent faults are faults that happen intermittently at a certain frequency, such as 
outdoor dampers getting stuck by snow periodically. As a general rule of thumb, abrupt faults are the easiest to detect; while incipient fault and intermittent fault are more difficult to detect due to their dependency on time.

Faults $F$, which consist of $F_{i}$ and $F_{e}$, firstly affect internal process parameters $\Phi$ by $\Delta \Phi$ and/or internal states $X$ by $\Delta X$. Eventually, faults affect measurable outputs $Y$ by a change of $\Delta y$. Note that $Y$ is also affected by measurement noise $N$. In building systems, most processes are closed loop with proportionally and/or integral acting controls $C$ which often make the change $\Delta y$ vanish over time, while causing a permanent shift in the control variable $U$. Therefore, for building FDD applications ideally control variables $U$ (e.g. supply air pressure), output variables $Y$ (e.g. indoor air temperature) and control deviation $e$ (e.g. difference to target supply air pressure) should all be measured and recorded for the FDD purposes. The overall system is shown in Figure 2-1.

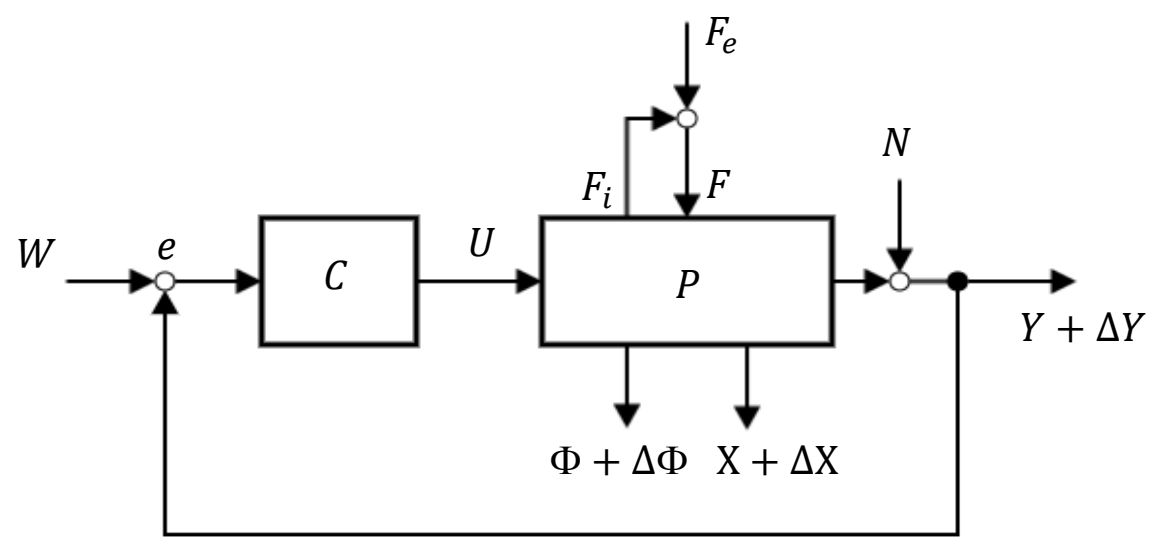

Figure 2-1 Sample technical process with faults

Faults can also propagate within the system through its process hierarchy. A lower level fault causing errors in outputs or control variables may propagate to a higher-level process through the hierarchy. On the other hand, a higher-level fault causing errors in output variables may also propagate to a lower level system's input. Fault propagation is further exaggerated by the 
complex two-way interactions between various building systems. To illustrate this, a lower level chiller fault can cause higher-level air handling unit to be not capable of providing enough cooling; and this higher-level fault of not enough cooling can cause a lower level thermal zone too warm. Because most building systems are composed of complicated and interconnected processes, this makes FDD applications in buildings especially challenging.

\subsubsection{Fault Detection}

According to S. Ding [20], the purpose of fault detection is "detection of the occurrence of faults in the functional units of the process, which lead to undesired or intolerable behaviour of the whole system”. Most of the modern fault detection methods are model-based, be it analytical models, rule models (if-then rules), or machine-learning models based on several measured variables. The fault detection process involves two steps:

1. Feed a combination of measured variables to a model, these variables include control inputs $U$ and states $\mathrm{X}$. Then it will generate features such as estimated parameters $\Phi^{\prime}$, predicted states $X^{\prime}$ when using analytical models, or some pure arithmetic values $A^{\prime}$ when using statistical procedures or machine learning models.

2. Apply change detection on features referencing nominal feature values, then if significant discrepancies are detected, the fault is detected. This decision can be reached by using simple threshold values, discriminant function, or probability calculations. For example, for states $X$, residuals $X^{\prime}$ minus $X$ can be compared against a threshold, and for some arithmetic values $A, A^{\prime}$ can be compared against the expected $A$ values with a null hypothesis test.

The fault detection process can be carried out either automatically using computer programs or manually through the observations made by an operator. An example of the conventional manual building fault detection process is the monthly energy usage intensity (EUI) analysis carried out by an energy analyst. There is a large variety of automated fault detection methods applied in 
building systems; popular methods include signal models, limit checking, parameter estimation, parity equations and principal component analysis, all of which will be further reviewed in the later sections.

In addition, the fault detection process also generates meaningful symptoms, which can be later used in the fault diagnosis process. Analytical symptoms $S_{a}$ are generated from automated fault detection algorithms and heuristic symptoms $S_{h}$ are generated through human observations. Table 2-1 summarizes the difference between the two symptom types. While symptoms containing physical meanings can be used in different fault diagnosis methods; symptoms generated with pure arithmetic values is often directly linked to a fault state in an integrated FDD system.

Table 2-1 Comparison of symptoms generated from fault detection process

\begin{tabular}{|l|l|l|}
\hline \multicolumn{1}{|c|}{ Symptom Type } & \multicolumn{1}{|c|}{ Analytical Symptom, $\boldsymbol{S}_{\boldsymbol{a}}$} & \multicolumn{1}{c|}{ Heuristic Symptom, $\boldsymbol{S}_{\boldsymbol{h}}$} \\
\hline Description & $\begin{array}{l}\text { Symptoms generated from models, } \\
\text { usually quantifiable and calculated } \\
\text { automatically on a computer/controller }\end{array}$ & $\begin{array}{l}\text { Symptoms generated from rule-based } \\
\text { reasoning, usually qualitative and } \\
\text { performed manually by operators }\end{array}$ \\
\hline Example & $\begin{array}{l}\text { Air temperature sensor measurement too } \\
\text { high; fan efficiency too low. }\end{array}$ & $\begin{array}{l}\text { Complaint of cold indoor condition by } \\
\text { occupant; visually observed water stain } \\
\text { on the ceiling. }\end{array}$ \\
\hline
\end{tabular}

\subsubsection{Fault Diagnostics}

Fault diagnosis is also called fault identification, fault isolation, or fault analysis by many researchers [28]. In this thesis, fault diagnosis means explicitly the process to identify and isolate the fault with as many details as possible such as its type, location, size and time. "Fault diagnostics" is the system that performs the fault diagnosis process. In the later sections, only 
"fault diagnostics" is used, since most building FDD work and the goal of this research are on developing fault detection and diagnostics systems.

Both analytical and heuristic symptoms can be used in fault diagnosis. It is advantageous to use a unified symptom representation for symptoms that enables communication between different AFDD models. This way the fault isolation process is natural to conduct [35] and can be integrated with different fault detection methods. Then the fault(s) can be determined based on the unified symptom representations using fault-symptom relationships, as shown in Figure 2-2.

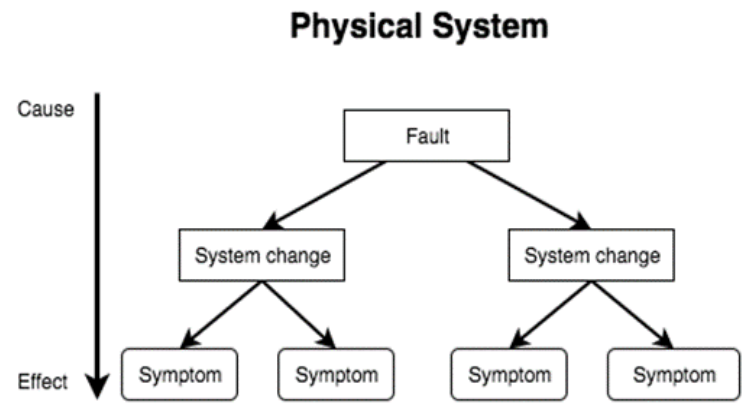

Physical System

Figure 2-2 Example of fault-symptom relationship [

\section{FDD System}

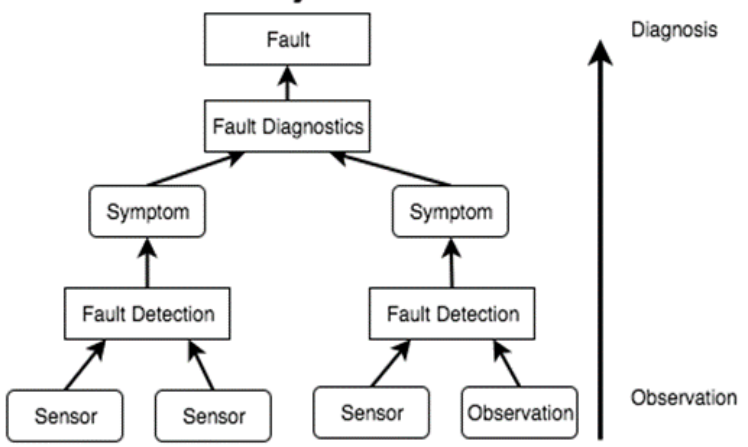

right: from symptom to fault inside a diagnosis system

Besides manual fault diagnostics through heuristic reasoning, there are two main automated fault diagnosis approaches: classification and inference. If fault-symptom causalities are known, inference methods such as binary reasoning can be adopted. Otherwise, classification methods without prior model structure knowledge such as statistical classification and artificial intelligence models need to be trained using experimental data. The term structure knowledge in this thesis means the structural relationships between faults, symptoms and observed/estimated variables. Note that machine learning techniques can be applied to both inference methods and classification methods; the difference is that for inference methods the causal relationships 
between faults and symptoms do not change through learning, while for classification methods, the causal relationships are not defined manually and have to be learned. It is also possible to use a hybrid method by applying both interference and classification methods, this way both the causal structure and parameter values can be learned from data. Table 2-2 shows a comparison between the different fault diagnostic methods.

Table 2-2 Comparison of approaches to automated fault diagnostic

\begin{tabular}{|l|l|l|l|}
\hline Method & \multicolumn{1}{|c|}{ Inference Methods } & \multicolumn{1}{|c|}{$\begin{array}{c}\text { Classification } \\
\text { Methods }\end{array}$} & \multicolumn{1}{|c|}{ Hybrid Methods } \\
\hline Description & $\begin{array}{l}\text { Infer possible faults } \\
\text { through causal } \\
\text { relationships with } \\
\text { symptoms }\end{array}$ & $\begin{array}{l}\text { Determine which state } \\
\text { the fault belongs to } \\
\text { using arithmetic } \\
\text { distances or } \\
\text { distribution }\end{array}$ & $\begin{array}{l}\text { Use the inference } \\
\text { method on known } \\
\text { causal relationship and } \\
\text { classification method } \\
\text { on others }\end{array}$ \\
\hline Examples & $\begin{array}{l}\text { If-then-else rules, } \\
\text { Bayesian Network, } \\
\text { fuzzy logic }\end{array}$ & $\begin{array}{l}\text { Support vector } \\
\text { machine, artificial } \\
\text { neural network }\end{array}$ & $\begin{array}{l}\text { Learnable decision } \\
\text { tree, learnable } \\
\text { Bayesian Network }\end{array}$ \\
\hline Advantages & $\begin{array}{l}\text { Easy to set up, usually } \\
\text { fast to compute, little to } \\
\text { no training data } \\
\text { required }\end{array}$ & $\begin{array}{l}\text { Require no prior } \\
\text { caustic knowledge, can } \\
\text { be set up automatically }\end{array}$ & $\begin{array}{l}\text { Capable of handling } \\
\text { both faults with or } \\
\text { without prior caustic } \\
\text { knowledge }\end{array}$ \\
\hline Disadvantages & $\begin{array}{l}\text { Require manual } \\
\text { construction for } \\
\text { different systems, } \\
\text { cannot identify } \\
\text { complex fault }\end{array}$ & $\begin{array}{l}\text { Slower to compute, } \\
\text { require large sets of } \\
\text { data }\end{array}$ & $\begin{array}{l}\text { Harder to implement, } \\
\text { still require some } \\
\text { manual input }\end{array}$ \\
\hline
\end{tabular}

\subsection{Overview of AFDD Research in Building Systems}

Automated fault detection and diagnostics in building systems is a challenging problem. Many of the processes inside a building are unmonitored or under-monitored such as infiltration and heat conduction through the building envelope. The complexity of AFDD is further aggravated by 
fault propagation. Also, due to different building functions and environmental conditions, system configurations in buildings tend to have large variations.

An ideal AFDD system should have those following characteristics [36]: low cost, reliable and

low false positive rates. Katipamula and Brambley [14], [17] also stated that future automated AFDD systems in buildings should:

1) Have no need for handcrafted AFDD algorithms. Many of the existing rule-based AFDD systems rely on predefined algorithms and need to be updated manually once system characteristic changes.

2) Become automatically configured. Since buildings contain many identical or similar systems, an automatically populated AFDD system can help reduce the configuration cost.

3) Have improved fault evaluation and decision support capabilities. Most AFDD research is focused on AFDD methodologies, which present little information to the building operators for decision-making. Improved decision support capabilities can enhance the usability of the AFDD systems.

The author of this thesis argues that building AFDD systems should also:

4) Contain an expandable system architecture so that new AFDD algorithms can be added modularly to accommodate different system configurations. Building systems and AFDD methods change over the building life-cycle, having an expandable AFDD system architecture allows it to be updated without a complete overhaul during a significant change in the building systems. 
Those requirements dictate the major design concepts of the AFDD system in this thesis. The selection of methods used in the AFDD system should be based on the process complexity, measurement availability, requirements for updates and other design objectives.

Most of the building AFDD systems in existing research are specific to an individual building system and often-times specific to a single fault detection or fault diagnosis method. This research aims to provide a generalized AFDD framework like the general diagnostic engine [36] so that methods from other AFDD research can be integrated to improve the overall performance and capability.

In their classic review papers, Katipamula and Brambley [14], [17] have classified building AFDD methods into three categories: quantitative model-based, qualitative model-based and process history based. This classification is similar to the three-part review articles on process AFDD research by Venkatasubramanian et al. [32], [34], [37]. In this work, the conventional way of AFDD categorizing is used: fault detection and fault diagnosis are discussed separately since this categorization can significantly improve the scalability of the proposed method. It is worth noting that many of the methods categorized in the later sections may be used as a hybrid method. For instance, Bayesian Network can be used as a black-box model or a grey-box model depending on how it is constructed and whether prior structure knowledge is given. 
More than 140 articles on building system AFDD have been reviewed for this thesis. This is not as comprehensive as some other reviews [38], [39], but the results are representative of the discipline. Figure 2-3 shows the number of articles reviewed sorted by year. Since the 2010s, effort on building AFDD R\&D has seen a steady increase due to the need to reduce global energy consumption and carbon emissions. Of all the 142 articles surveyed, 39 are only focused on fault detection or anomaly detection, while the other 103 articles propose fault detection and diagnostic methods. A detailed review of different fault detection and fault diagnostic methods will be provided in the following sections.

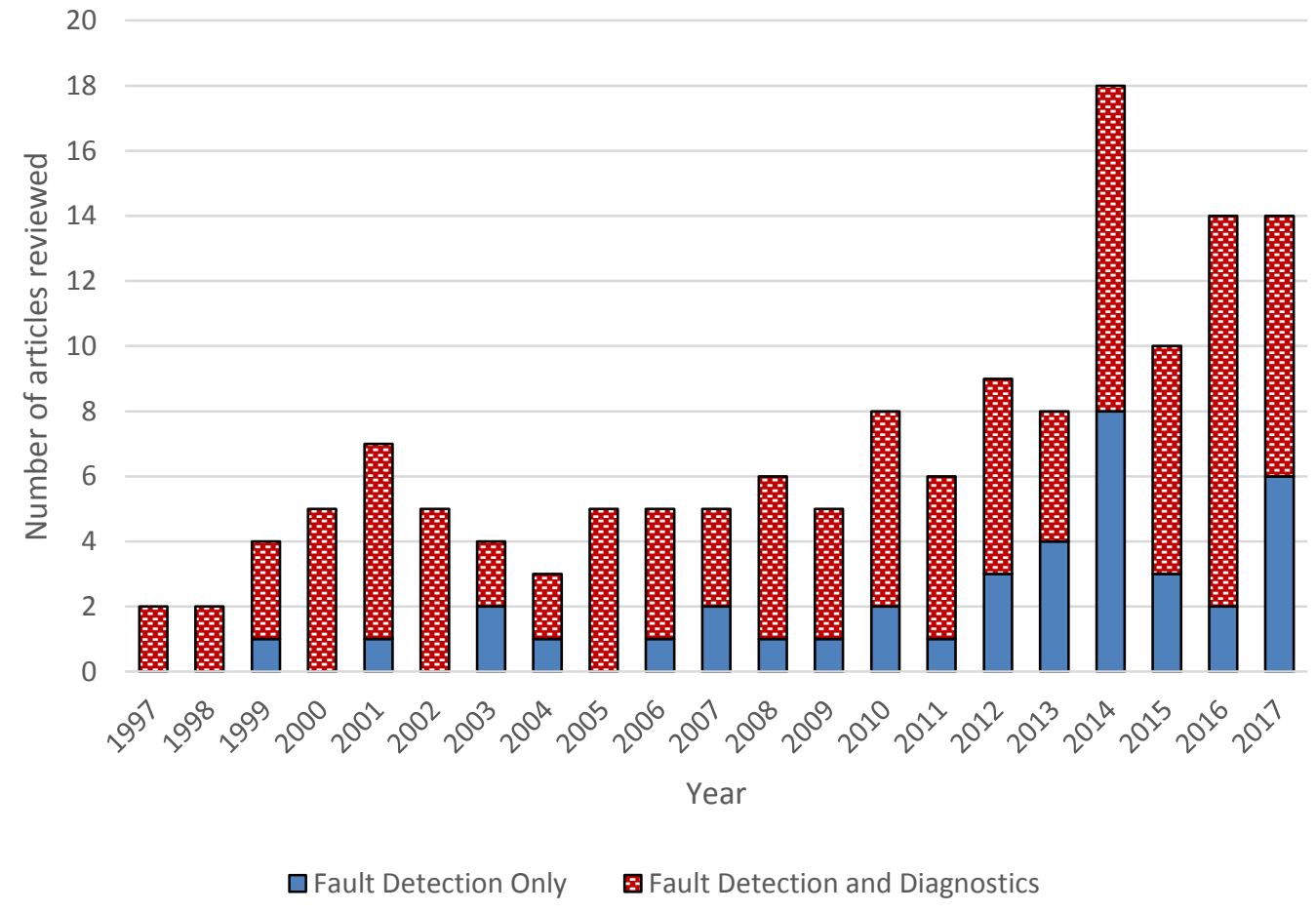

Figure 2-3 Number of articles per year surveyed in this literature review 


\subsection{Fault Detection in Building Systems}

As mentioned previously, the task of fault detection is to detect faults within building systems, without necessarily identifying and isolating them. In a generalized FDD framework, fault detection process is also responsible for generating symptoms for the fault diagnosis process. In conventional control theory research, fault detection methods are usually classified into three categories: limit checking, signal analysis and model-based analysis [40]. The model-based analysis is most commonly used in building AFDD research nowadays and contains many subcategories such as qualitative models, first principle models, and grey-box models. This section provides a review of different fault detection techniques in building systems. Since many scientific papers do not explicitly separate fault detection and fault diagnosis tasks, some of the contents reviewed may overlap with later sections.

\subsubsection{Limit checking and trend checking}

Limit checking is a classical fault detection method and commonly applied in most building digital control systems nowadays. This form of fault detection typically works by checking if a single sensor variable is inside a static or adaptive range. Trend checking works by checking if the first derivative of the sensor variable is within a certain range. Trend checking can be commonly found in machinery but less commonly in building applications. Other methods using single sensor data such as change detection and plausibility checks [31] are also available. Trend checking and limit checking analysis are fast responding and easy to implement, but they have limited capabilities in building fault diagnosis due to limited sensing capability and fault propagation in complex building systems. Still, these methods are suitable for critical system 
faults [28] that are directly monitored, such as fire alarms, high carbon monoxide level or frosting on AHU components. Examples of using limit checking in building system fault detection include usage of statistical models to establish limit thresholds [41], and thresholds created using expert knowledge [42].

\subsubsection{Signal analysis}

Signal analysis methods focus on capturing oscillating or periodical behaviour of the processes originated from classical control theory. These processes could contain periodical signal(s) and/or stochastic signal(s) that can be used in signal models as single or multi-variable analysis. Many of the AFDD applications using periodical signal analysis such as bandpass filtering and Fourier analysis have been used in machinery and electronic systems [28], [43]. Since most of the signals inside building systems are non-stationary and stochastic, signal analysis methods are often used in conjunction with other process model based methods, such as an application in VAV systems using a combination of wavelet analysis and artificial neural network [44].

\subsubsection{Model-based analysis}

Process model-based fault detection uses the dependencies between measured variables to detect possible changes caused by faults. Both analytical models and causalities (if-then-else) can be used to describe those dependencies. The analytical relations could be obtained from first principals, simplified physical models or pure statistical models. The causalities can be derived from expert experience, qualitative models or machine learning methods. Most of the AFDD 
applications in building systems use model-based analysis due to the limited amount of sensing capabilities and complex interactions between the measured variables.

According to Wen et al. [45], models used for building AFDD and control applications should:

1) Use commonly available measurement sources. Some novel sensors such as occupant count sensor and infrared cameras can provide much-needed insight into the operation of a building. However, these sensors not widely available yet, so reliance on these sensors could limit the applicability of the fault detection method.

2) Be easy to update. Characteristics of a building system can change over time, repurposing of a room, change of sensor location, or change of AHU components can make existing AFDD process outdated. So, it is crucial for the AFDD process to be quickly updated to remain functional.

3) Be capable of predicting states in the near future. The functionalities of AFDD and model predictive controls (MPC) are often intertwined, they both use process models, for AFDD is to compare parameters and state outputs, for MPC is to predict future states given different control inputs. Thus, if the AFDD model and MPC model can be integrated, the computation load on the controller can be significantly reduced.

Table 2-3 shows the comparison between different types of process models used in AFDD applications. While all of the process models can act as observers (predicting and comparing measurable states), only white-box model and grey-box model are capable of acting as parameter estimators at the same time. However, white-box models are usually too complicated or too costly to update their parameter estimates recursively. A detailed description of each process model type will be discussed in the following section. 
Table 2-3 Comparison of different model-based fault detection methods

\begin{tabular}{|c|c|c|c|c|}
\hline $\begin{array}{c}\text { Process Model } \\
\text { Types }\end{array}$ & $\begin{array}{c}\text { Model } \\
\text { Complexity }\end{array}$ & $\begin{array}{c}\text { Parameter } \\
\text { Estimation }\end{array}$ & Update Method & $\begin{array}{c}\text { Update } \\
\text { Complexity }\end{array}$ \\
\hline $\begin{array}{c}\text { Qualitative } \\
\text { Model }\end{array}$ & Low & No & Manual & High \\
\hline $\begin{array}{c}\text { White-box } \\
\text { Model }\end{array}$ & High & Yes & Manual/Automatic & Low \\
\hline $\begin{array}{c}\text { Grey-box Model } \\
\text { Black-box } \\
\text { Model }\end{array}$ & Low-Medium & Yes & Automatic & High \\
\hline
\end{tabular}

\subsubsection{Qualitative models}

The qualitative analytical model uses a qualitative representation of the variables and relations to represent the behaviour of the process. For example, a qualitative model can be described as: during heating seasons with other internal heat gain $(\mathrm{G})$ sources remain constant, an increase in lighting load (LE) will result in more internal heat gain (G), and can be written as $G \propto_{Q_{+}} L E$. This notation simply means LE increasing causes $\mathrm{G}$ to increase while all else being equal. Details about this method can be found from the qualitative simulation and modeling theories [46][47]. Qualitative models fit for cases with high measurement noise or when only qualitative observations are available, such as human observations [37], [48].

There are two types of qualitative models for AFDD: rule-based models built on expert knowledge or first principles, and qualitative analytical models purely based on first principles. Applications of qualitative analytical models in building AFDD are relatively rare; one example is the qualitative analytical model developed by Frontera et al. for AHUs [49].

Rule-based, or the so-called "expert" AFDD, systems are one of the most widely researched and developed AFDD methods used in building systems. They are easy to develop, transparent and have the ability to reason under uncertainty. On the other hand, this method is very specific to 
each individual system and often difficult to update or change. Examples of those systems including the APAR system developed by Schien et al. [50], the air handling unit (AHU) InFO system by Burton et al. [51], and many other commercial FDD products.

\subsubsection{White-box models}

White-box models are quantitative analytical models based on first principles [34]. Those models usually have a good representation of the actual process, have the advantage to simulate fault states and are good at representing transient states [37]. However, they are often too complicated to implement and compute, require a lot of data input which may not always be available, and very hard to calibrate/recalibrate due to a high degree of freedom [14]. These models are usually used as forward models in which the parameters are predefined based on design information. Recalibration is possible but usually limited to a small parameter space. Unfortunately, in many cases, the parameters inside the constructed building may vary from the design under different operation conditions. This would make white-box models impractical without sufficient computation power, especially when up-to-date parameter estimation is needed. Numerous detailed and simplified physical models have been developed for HVAC components and AHU [52][53][54]. For whole building and zone responses, most of the white-box models used in AFDD research are based on the existing building energy simulation tools [55]-[57], while there is still work to be done to develop more simplified models to improve overall performance and decrease model complexity. Most of the white-box model implementations in building AFDD 
use parity equation (comparison between model prediction and actual measurement), while the possibility of using parameter estimations for fault detection is rarely discussed.

\subsubsection{Grey-box models}

Grey-box models are analytical models loosely based on first principles, in which the model parameters can still be traced to the process's physical response [17]. Compared to white-box models they are faster to compute and easier to calibrate; while compared to black-box models they are more robust and can be used for parameter estimation. On the other hand, formulating grey-box models requires expert knowledge and extensive measured data are required to train their model parameters [17]. Luckily, most of the processes inside buildings or zones are structurally similar, so common grey-box models can be formulated relatively easily. Grey-box models have seen numerous implementations in both control applications and AFDD systems for building systems and are typically implemented as inverse models in which the parameters are learned and updated from operation data. While many of the grey-box models that have been developed for HVAC systems and its components [58]-[60], some research has been performed in the whole building and building zone grey-box models [61]-[64]. Many of the grey-box models used to represent a whole building or zone thermal behaviours are based on the work done by Braun and Chaturvedi [62] and have been proven very effective in both AFDD and model predictive control applications [65]-[69].

\subsubsection{Black-box models}

Black-box models are statistical or non-statistical models in which the model parameters convey no physical meaning [17]. These models can be compelling due to model flexibility and usually use operation data to train its parameters. However, often times black box models can only act as 
observers since the estimated parameters provide little information about the actual physical process. The fast-growing machine learning field has led to a growing trend of research work on using black-box models in AFDD applications. Some common examples of the black-box models include polynomial curve fit, artificial neural network (ANN), principal component analysis (PCA), support vector machine (SVM) and others. Those models require little prior knowledge of the system to set up, are easy to develop, and can be updated. However, they usually provide little information beyond state observer and are very specific to each system. Black-box are more effective in HVAC and its components since those systems are usually equipped with enough sensors to compare with the observer [41], [54], [70]-[78], while as in other building systems and zones they are often applied in fault detection systems with limited capabilities for automated fault diagnosis [55], [79]-[82]. Some black-box models are directly used to diagnose faults without detecting them first. These methods are called "one step diagnostics" in this review and will be discussed in the next sections.

\subsubsection{Review of fault detection methods in building systems}

Figure 2-4 shows the composition of various types of fault detection methods from the literature survey. Black-box models are most common, accounting for more than half of the articles. Greybox models come second due to their flexibility. 


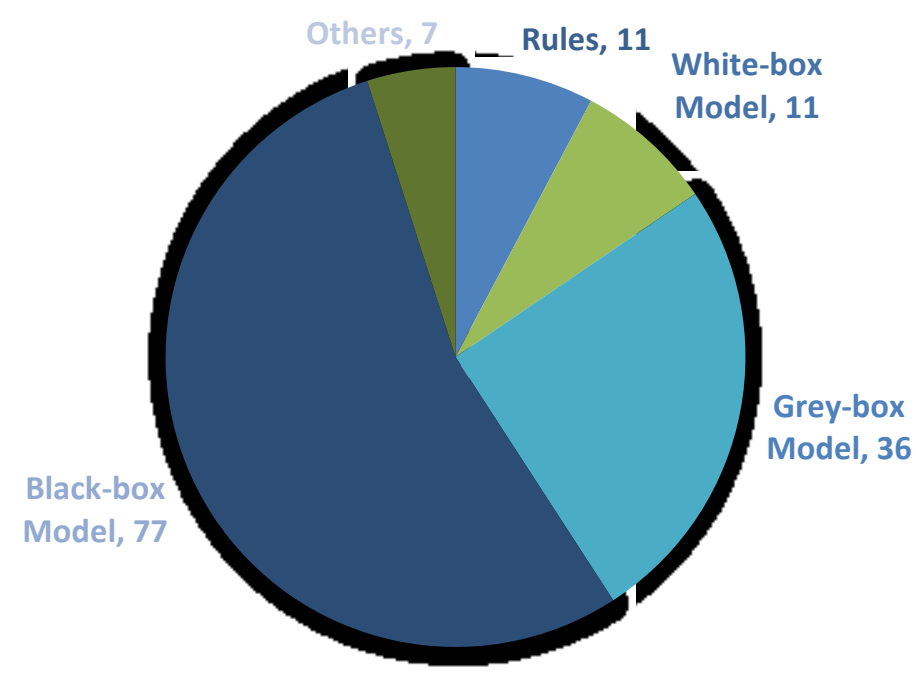

Figure 2-4 Survey of different fault detection methods

Interestingly, when splitting the surveyed articles by the year 2010, the compositions of articles become different, as seen in Figure 2-5. The number of articles using Black-box models to detect faults has significantly increased after 2010, while the popularity of other methods remains relatively consistent. This can be explained by the increasingly powerful statistical models and machine learning algorithms, which became generalized enough to be applied to building systems. Among these black-box models, the most popular ones are PCA (17 articles), variations of autoregressive models (AR) (5 articles) and variations of ANN (4 articles). The popularity of these models agrees well with the AFDD research from other disciplines [37] due to their flexibility and robustness. 


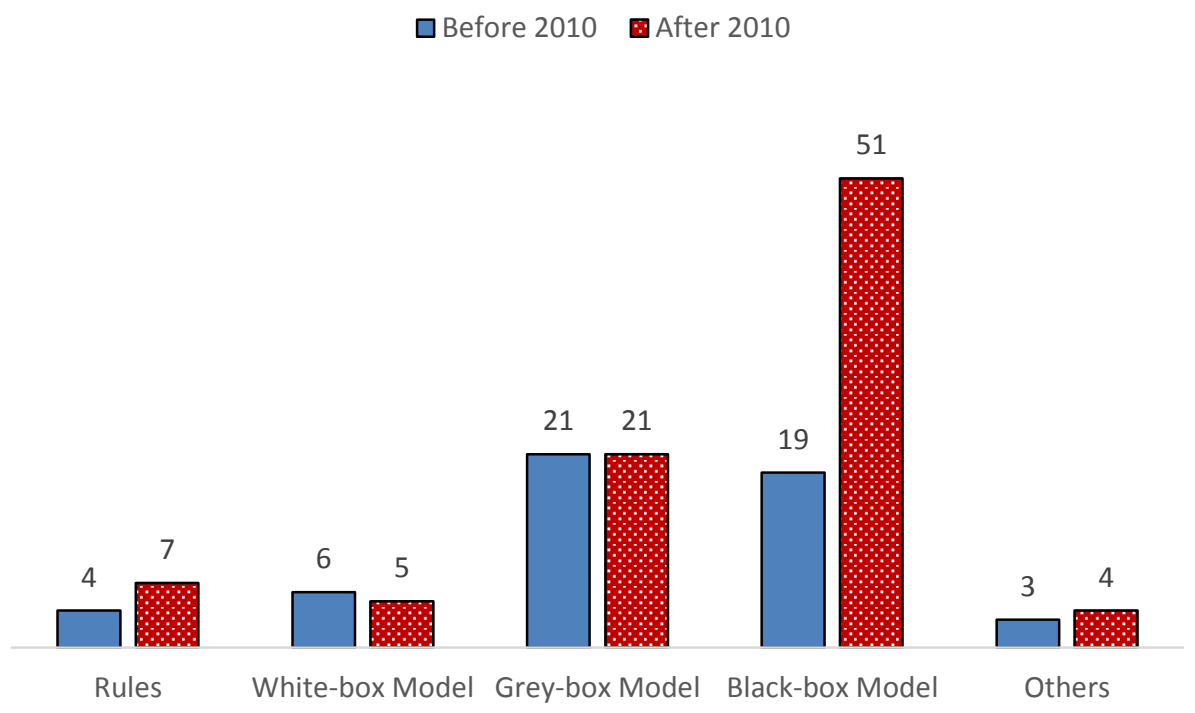

Figure 2-5 Comparison of different fault detection methods before and after 2010

\subsubsection{Summary}

While there are numerous fault detection methods available for building systems, grey-box models are probably the most suitable candidate for this task. Grey-box models can perform both state and parameter estimation, providing more insight into the system. They are easy to set up and simple enough to update and run in real-time even inside a controller, but still adequate to capture the physical dynamics of the system. This thesis will focus on fault detection using established grey-box models from previous research.

\subsection{Fault Diagnostics in Building Systems}

According to Isermann [28], the fault diagnosis process can be classified into two categories: inference methods and classification methods. Inference methods are applicable when the causalities between faults and symptoms can be expressed explicitly, while classification methods can be trained experimentally when the causalities are not apparent [28]. The following 
section mainly follows the conventional fault diagnosis research in the field of control theory.

The fault diagnosis process typically uses analytic and heuristic symptoms with predefined faultsymptom relations and/or classification methods with forward and backward chaining.

In many theoretical FDD papers, the tasks of fault detection and diagnostics are often separated to ensure the modularity and expandability of the system [35], [36], [83]. Many modern building FDD articles do not explicitly separate these two components, especially for methods using statistically driven models such as ANN [84]-[86] and PCA [87]-[89]. Integrated fault detection and diagnostics processes could work well on individual subsystems but lack the flexibility of incorporating other fault detection or fault diagnostics algorithms. They also lack the ability to communicate between different systems, due to the overfitting tendency of black-box models. This might change though, given the development of data collaboration for building AFDD and reinforced deep learning. In the near future, separated fault detection and fault diagnostic subsystems still is a more flexible option. It allows different fault detection and diagnosis algorithms to be combined and altered during the building operation, which can greatly improve the usability and expandability of an AFDD system.

\subsubsection{Inference method}

The most basic form of the inference method is a fault tree based on a single decision tree and multiple binary relationships. The symptoms are generated from Boolean equations in binary form during fault detection. The "expert" AFDD systems, mentioned in the qualitative models in the previous section, typically uses predefined fault trees and cause-effect relations between the symptoms and faults to perform the fault diagnosis task [50], [90]. Fault trees only work in the manner of discrete-event, and for continuous faults such as incipient faults and intermittent 
faults, approximate reasoning can be used. Approximate reasoning can be carried out by fuzzy logic or probabilistic reasoning in the form of forward chaining or backward chaining. Several building AFDD applications have used fuzzy logic [72], [91], [92] to diagnose faults.

Due to the requirement of prior knowledge, inference method may not be suitable for all AFDD implementations, but it is still very powerful for building systems since most of the faultsymptoms relationships can be derived from first principles or expert knowledge. This makes the inference method the most used approach from the survey articles, as 53 out of the 102 reviewed papers chose this approach. However, this does not mean inference method is the superior choice, as larger building systems such as AHU may have an enormous amount of faultsymptom causal relationships that need to be manually defined. Furthermore, the lack of ability to be updated automatically means inference models have to be maintained manually during operation changes or system retrofits. This limits the usability of the inference methods, but still, easy to use, no training data requirement and intuitiveness mean they are often the first choice when developing new AFDD systems.

\subsubsection{Classification method}

Classification methods not only can be used when the structure knowledge between the faults and symptoms is unknown, but also remain applicable even when partial structure knowledge is defined. Operation data including fault cases as well as prior knowledge can be used to train the classification algorithms. Common classification methods used in AFDD systems include decision tree, Bayesian Network classifier, geometrical classifier and artificial neural network classifier. One of the most commonly used classifiers in building AFDD is the artificial neural network classifier (ANN) [86], [93]-[96] and Bayesian Network (BN) classifier [76], [82], [97]- 
[99]. Geometrical classifiers have also been used in some fault diagnosis applications [96], [100], [101]. Most of these classifiers come from computer science, and statistic research have been proven very effective in AFDD research [28], [32]. However, one of the major shortcoming for many classifiers is the requirement for a large set of data for training. This training data set almost always requires data from faulty operations, which may hard to come by since not all faults can be easily recreated without causing permanent damage. Thus, classifiers may be suitable for individual equipment since its faults can be emulated and analyzed before its production.

In 49 of the surveyed articles which used classification methods, Bayesian Network (BN) and its variations are the most commonly used, accounting for nine in total. It is followed by ANN and its variations, which are used in eight articles. One strength of BN comparing to other classifiers is that is can be constructed using prior knowledge similar to inference models, while at the same time capable of being updated using operation data [102]. This becomes the hybrid of the two categories and may become very useful to building systems.

\subsubsection{Direct vs indirect diagnostics}

In some AFDD research, the fault detection step and symptom generation step are omitted, which means a black-box classifier is capable of diagnosing faults based on direct measurements from sensors. This is common when using black-box classifiers - the classification model is trained from data anyway, so it does not require symptoms to be generated beforehand. In fact, this direct approach has become increasingly popular as the classifier methods become the dominant choice for fault diagnostics, as shown in a comparison in Figure 2-6. 
Even though the direct approach can simplify the fault diagnostic process and potentially increasing its accuracy by avoiding modelling error incurred during the symptom generation step, this approach can cause several critical issues. These issues include different AFDD methods cannot run in parallel and share information, and it is impossible to obtain a unified fault representation from symptoms generated by different methods. This is a lost opportunity to obtain more information from various AFDD methods and is against the design philosophy of this project.

On the other hand, the indirect diagnostics approach follows the classic fault detection symptom generation - fault diagnostics procedures proposed by Isermann, as described in Section 2.1. This allows the possibility of integrating other fault detection or fault diagnostics methods by using symptoms to transport information between different methods. However,

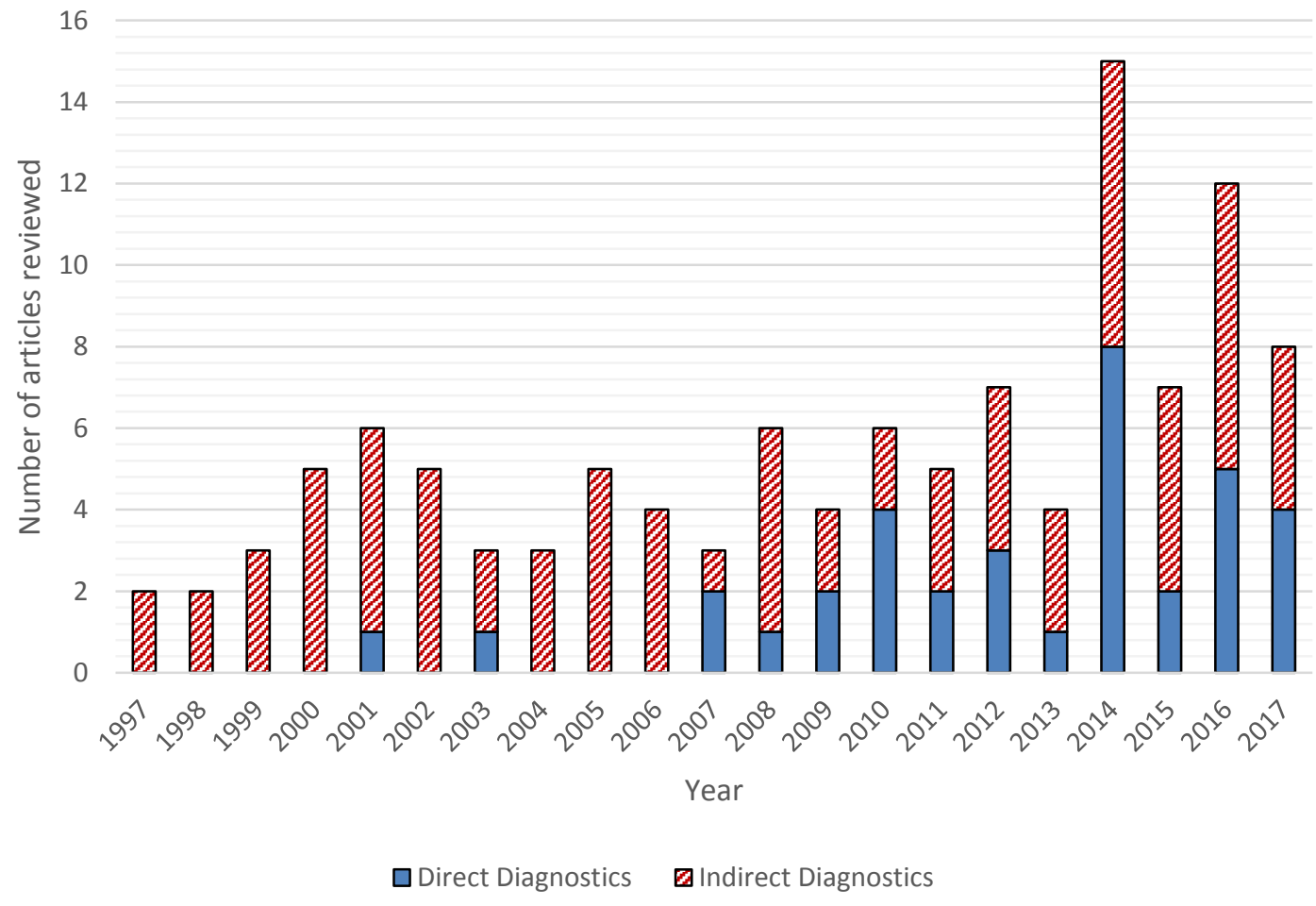

Figure 2-6 Comparison of direct and indirect diagnostics approach by year 
potential problems of modelling errors introduced by the fault detection process, and how to quantify symptoms are the problems that need to be addressed.

\subsubsection{Single-agent vs multi-agent diagnostic system}

In most AFDD research in buildings, all fault detection and fault diagnosis tasks are performed simultaneously at a central location - usually a centrally located server connected to the building automation system, or a computer embedded on a piece of equipment. This approach often works well for single equipment or building system but lacks scalability when comes to large-scale interconnected systems. Another issue brought by this single agent approach is the increased traffic induced to the existing congested building control network, as a centrally located complex AFDD process usually requires a significant amount of real-time data.

So instead of a central AFDD process, its tasks can be dissected into smaller tasks performed by multiple distributed agents. These agents will then communicate with each other with extracted information instead of raw data - which leads to a significant reduction in network traffic. This design also allows different fault diagnosis agents to focus on different local systems, and to communicate if fault propagation is suspected. Thus, the framework of the proposed AFDD system in this thesis uses a multi-agent diagnostic approach as its backbone.

\subsubsection{Summary}

Inference methods are easy to setup, but if prior knowledge is insufficient to set up the model, it might be better to use classification methods and train the diagnostic model with process history. However, the challenge with the data-driven fault diagnosis process in building systems is that it 
is often impractical to create fault cases inside building systems for statistical model training purposes due to the following reasons:

1) Building systems are usually required to be online constantly without backups.

2) It is often hard or impossible to create fault cases without causing permanent damage to the system.

3) Fault cases have to be recreated after systems' behaviour changes due to equipment or occupant changes.

This challenge might change thanks to active research in how to integrate AFDD applications in building commissioning period and using building simulation tools to create artificial fault data, but these topics are not the focus of this research. In this project, classification method with prior fault-symptom knowledge during the setup such as Bayesian network will be used, since it provides advantages from both the inference method and the classification method. In addition, the author will apply a two-step AFDD process with a distributed structure to enhance modularity and scalability.

\subsection{Fault Evaluation}

As concluded in the review by Katipamula and Brambley [17], researchers developing AFDD methods often overlook their implications on fault management - how to meaningfully present AFDD results to the building operators to make decision-making faster. Katipamula and Brambley [17] proposed to include fault impact assessment in AFDD systems to provide quantitative metrics to the operators, which can help the operators prioritize their work schedule. Still after a decade, according to the recent survey by Kim and Katipamula [103], only 28 out of the 197 articles provided fault impact estimations regarding energy and cost. Moreover, more 
than $80 \%$ of the 28 articles reporting fault impacts were focused on individual mechanical equipment such as heat pumps and cooling towers. Only O’Neill et al. [104]included whole building fault impact assessment as part of their study when using EnergyPlus as an anomaly detection tool. There is a lack of research on providing a systematic approach to different levels of faults inside building systems.

Interestingly, recent years saw the development of numerous fault models inside building performance simulation (BPS) tools. For instance, a comprehensive list of fault models was developed with OpenStudio Measures [105], and more new fault models are becoming available in EnergyPlus [106]. Combined with the versatility of BPS tools to change their numerous inputs, this presents a unique opportunity to utilize BPS to simulate and assess fault impacts in buildings in an adaptable manner.

Another approach to evaluating fault impacts is to directly compare measurable metrics before and after the fault. Those methods can be created based on existing retrofit analysis procedures such as ASHRAE Guideline 14 [107] since they both analyze the effect of a parameter change within a system by directly comparing the measured metrics. If the metric of interest is directly observable, this approach can provide reliable analysis. However, it requires sufficient data to be collected after the fault event, and this data cannot be used to reasonably predict future impacts if the fault is not remedied.

The third approach is to apply grey-box models for fault evaluation. These grey-box models could be the same models used for AFDD, but in this case applied to forecasting future impacts of the faults. In addition, it may not contain all the parameters that can be altered to reflect the changes caused by a fault. Grey-box models are less reliable than BPS modelling, but may become handy when a calibrated BPS model is not readily available, especially for less complex 
symptoms such as decreased equipment efficiency. Table 2-4 shows a comparison between using BPS or statistical model to evaluate fault impacts.

In this thesis work, a fault evaluation scheme using BPS modelling is proposed. This process is integrated with the regular FDD tasks inside the proposed framework and is used to provide quantitative evaluations of the diagnosed faults to the building operators.

Table 2-4 Comparison of fault impact evaluation approaches

\begin{tabular}{|c|c|c|c|}
\hline Name & Statistical Modeling & BPS Modeling & Grey-box Modeling \\
\hline$\underline{\text { Pros }}$ & $\begin{array}{ll}\text { - } & \text { Reliable } \\
\text { - } & \text { Little prior inputs }\end{array}$ & $\begin{array}{l}\text { - } \begin{array}{l}\text { Capable of predicting } \\
\text { future impacts }\end{array} \\
\text { - } \quad \text { Can evaluate impacts } \\
\text { that are not measured } \\
\text { - } \quad \text { Reliable }\end{array}$ & $\begin{array}{l}\text { - Capable of predicting } \\
\text { future impacts } \\
\text { - Easy to update }\end{array}$ \\
\hline$\underline{\text { Cons }}$ & 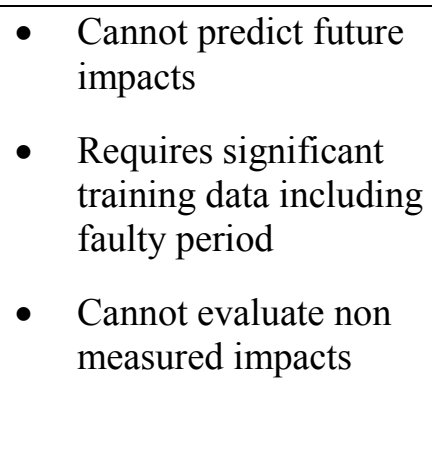 & $\begin{array}{ll}\text { - } & \begin{array}{l}\text { Requires expert } \\
\text { knowledge }\end{array} \\
\text { - } & \begin{array}{l}\text { Require calibrated } \\
\text { model }\end{array} \\
\text { - } & \text { Hard to update }\end{array}$ & 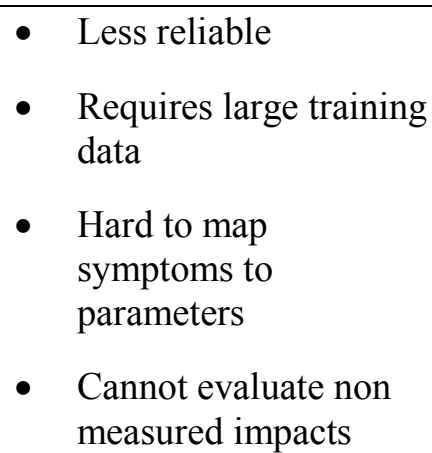 \\
\hline
\end{tabular}

\subsection{Existing Building FDD Products}

This section provides a brief survey of existing building FDD products on the market. Basic fault detection capabilities based on limit checking was provided since the 1980s when direct digital controls began to gain popularity in buildings. However, it is not until the 2000 s that we saw the release of rule-based expert systems as fault diagnosis guides for the building operators. More 
advanced AFDD products using statistical model and machine learning methods are still rare in recent years, despite their popularity in building FDD research.

Table 2-5 shows a list of commercial FDD products surveyed in this technology review. Many of the FDD services on the market still relies on experts to manually analyze and compile operation data to provide a possible diagnosis (expert supervision). This approach often provides reliable results but is not cost effective and scalable to be widely adopted by the building industry. On the other hand, although nowadays most of the commercial building AFDD products are still rulebased, some advanced products based on state of the art research has emerged from both smaller startup companies and traditional BAS and BEM vendors.

Besides the emergence of data-driven AFDD products, some infrastructure problems remain unresolved. For example, the problem of how to integrate data from different buildings used for training machine learning models is a relatively new research topic, which has been investigated by some researchers [108], [109]. In addition, integration of design specifications from building information modelling (BIM) technologies has also been discussed recently [110], [111]. These infrastructure problems remain essential to make future building FDD technologies realistic. 
Table 2-5 Survey of existing FDD products

\begin{tabular}{|c|c|c|c|}
\hline Name & Building Type & Company & FDD Method \\
\hline Analytika Pro & Large Commercial & Cimetrics & Expert supervision \\
\hline Attune & Large Commercial & Honeywell & Expert supervision \\
\hline Building Analytics & Large Commercial & Schneider Electric & Benchmarking \\
\hline Cite-AHU & $\begin{array}{c}\text { Medium/Large } \\
\text { Commercial }\end{array}$ & NIST & Rule-based system \\
\hline Clockworks & $\begin{array}{l}\text { Medium/Large } \\
\text { Commercial }\end{array}$ & KGS Buildings & Expert supervision \\
\hline $\mathrm{DABO}$ & Large Commercial & $\begin{array}{c}\text { NRCan/ADMS } \\
\text { Technologies }\end{array}$ & Rule-based system \\
\hline Golden Standard & Large Commercial & Delta Electronics & Benchmarking \\
\hline PACRAT & Large Commercial & Facility Dynamics & Rule-based system \\
\hline Panoptix & Large Commercial & Johnson Controls & Benchmarking \\
\hline SciWatch & Large Commercial & $\begin{array}{c}\text { Scientific } \\
\text { Conservation }\end{array}$ & ANN \\
\hline SkySpark & Large Commercial & Skyfoundry & Rule-based system \\
\hline Tririga & Large Commercial & IBM & Unknown \\
\hline
\end{tabular}




\subsection{Summary}

This chapter provided a review of fault detection, diagnostics and evaluation in building systems. It included fundamentals of FDD, characteristics of different fault detection and fault diagnostic methods, and their implementations in building systems. Basic definitions of fault, symptom, fault detection and fault diagnostic were detailed. The formulation of the proposed framework is based on this literature review which includes a survey of over 140 research articles. Existing building AFDD research and products were also categorized, compared and summarized. The proposed AFDDE framework splits fault detection and fault diagnostics tasks into separate processes. Grey-box models will be used to generate extra information about building systems and then generate symptoms during fault detection. Bayesian Network-based fault diagnostic method is selected due to its versatility, ability to use prior structure knowledge and less tasking requirement on training data. The lack of fault evaluation was also identified, and model-based fault evaluation will be implemented in the proposed method. The next chapter details the methodology of the proposed AFDDE framework, and how each component of it can be generalized to other related research topics. 


\section{Chapter: Methodology}

This chapter introduces the proposed AFDDE framework, the selected methods are derived from the previous literature review. First, an overview of the framework is provided, followed by detailed formulations of different functioning agents used in this framework. Note that this chapter provides theoretical formulations of the framework components, while details of zonelevel and AHU-level implementations will be explained along with the case study results in Chapter 4. Several generalizable novel contributions such as parameter estimation using constrained Dual Extended Kalman Filter, Dynamic Bayesian Network for fault diagnostics, and model-cluster-reduce method for BPS model order reduction are also introduced in this chapter.

\subsection{Framework Architecture}

Based on the reviews in Chapter 2, the author of this thesis opted to develop a distributed FDDE framework. The advantages of adopting a distributed system include the flexibility over different building systems, the capability of mix different fault detection and fault diagnostics algorithms, and the ability to run on different time steps inside different devices to reduce network traffic and computation requirements. Different functional agents need to be established to distribute work required to perform the FDDE work, and shared objects need to be created to enable communication between different agents.

Figure 3-1 provides an overview of the proposed framework. This distributed framework is composed of three types of functioning agents: fault detection agent, fault diagnostics agent and fault evaluation agent. The responsibilities of different agent types are:

- Fault detection agent performs fault detection and extracts additional information about the building system that is not directly measurable from the sensors. Fault detection 
agents also generate symptoms using signal or process models. It is preferable to implement this type of agent locally (inside the controller) to achieve faster response and minimize network overhead. In cases where the monitored system is not time-critical, and the detection algorithm is computationally heavy, the agents can be implemented remotely at a slower scan rate.

- Fault diagnostics agent collects symptoms, then processes them through fault diagnostic algorithms. Since fault diagnostic algorithms in building systems are usually quite sophisticated, they need to be implemented on remote servers instead of local controllers. A customized Dynamic Bayesian Network (DBN) is built to perform fault diagnosis tasks due to its expandability and ease to set up.

- Fault evaluation agent: uses symptoms and faults generated from the other two agents to estimate the impacts of faults based on their symptom severity. The results are represented in quantifiable metrics such as energy usage. This process usually involves

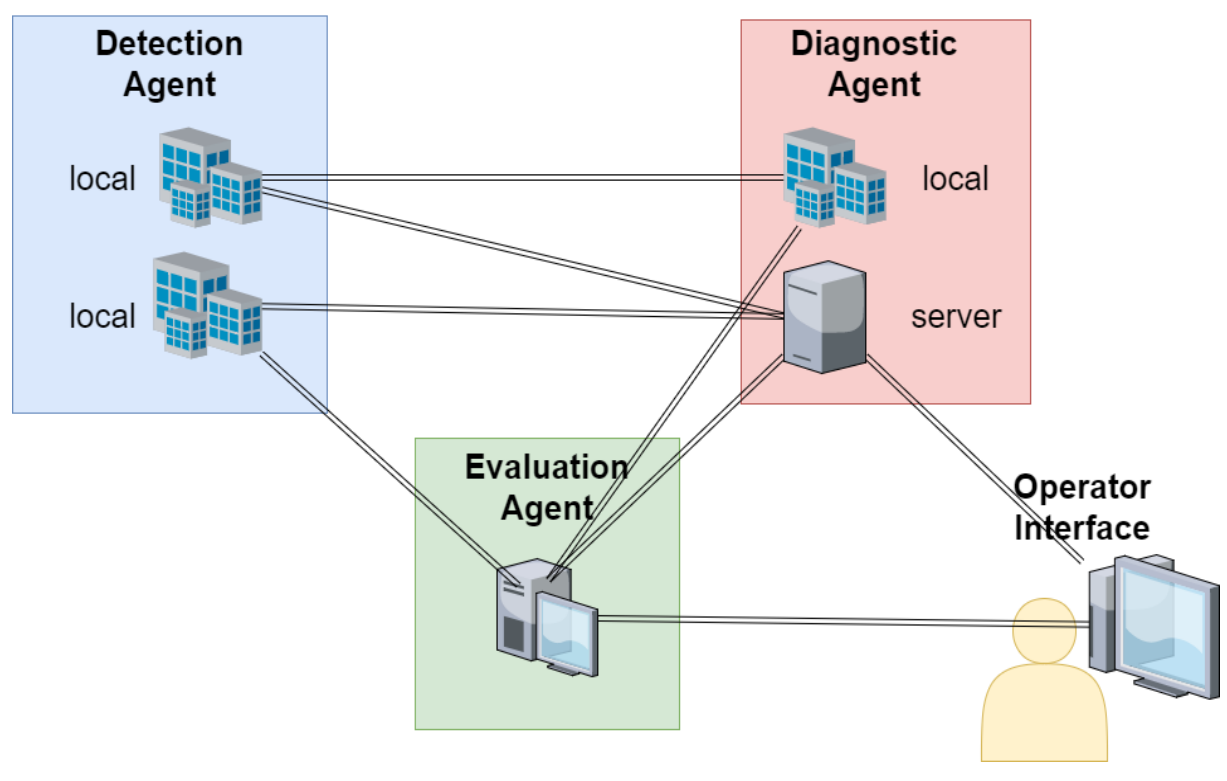

Figure 3-1 Overview of the distributed fault detection, diagnostics and evaluation framework 
heavy model simulations, and only needs to be done at a much lower frequency, usually daily or weekly. Thus, it should be implemented on a remote server or a cloud-based service.

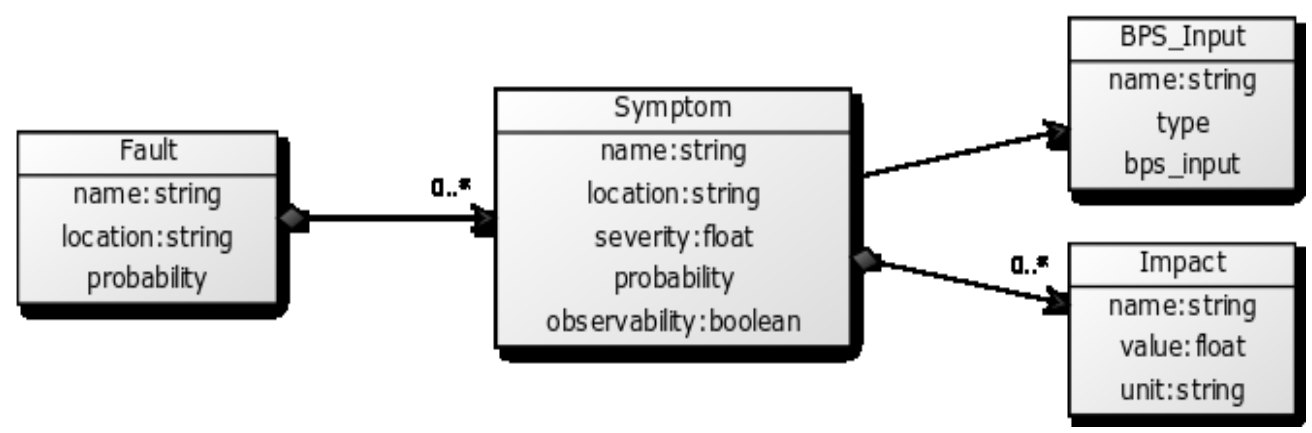

Figure 3-2 Symptom, fault and impact used in the framework

Besides three types of agents, there are also three main types of objects used in this multi-agent system: symptom, fault, and impact. These objects are used to establish communication between the agents and exchange information. Figure 3-2 shows an overview of these objects. Qualitative causal relationships between faults, symptom, and impacts are also established. The "0..*" on the figure denotes many-to-many relationship, i.e. several faults might have cause multiple symptoms to happen.

Symptoms are anomalies detected and quantified by the fault detection agents. They are analogous to symptoms observed during medical examinations used for diagnosis evidence. Besides variables such as location, name, and type, they are described by two quantitative variables - severity and probability. Symptom severity, denoted by z, is a continuous value used to represent how severe the symptom is, and how much it deviates from normal operation. Symptom probability, denoted by $S$, is a probability value in the range of $[0,1]$ representing how 
likely an unwanted symptom is present in a building system. Examples of symptoms include low air temperature inside a supply air stream, or high $\mathrm{CO}_{2}$ concentration inside a room. Faults, as defined in the previous chapter, are unwanted deviations from normal operation inside building systems. As mentioned in the literature review section, they are the direct cause of symptoms. Besides their names, locations, they are quantified by fault probability $F$ representing how likely the fault is True and is affecting the building system.

Impacts are effects of faults in building systems that are of interest to stakeholders. They are important to provide quantitative assessments of faults to the building operators to help them prioritize their tasks. In this research three types of impacts are used to evaluate faults - energy, cost, and comfort. Energy impact uses source energy in $k W h$, cost uses Canadian dollar \$, and comfort is quantified by percentage dissatisfied with the indoor environment (PPD). This thesis adopts probabilistic representations of fault states and symptom states. For example, instead of a symptom described as in a deterministic Positive state, it is described as $99.9 \%$ Positive and $0.1 \%$ Negative. The rationale behind this approach is that it provides better flexibility over uncertainties including imperfect models and partial operation observability, which will be examined in detail throughout this chapter. The author will not spend time debating the appropriateness of using probabilistic theories for fault diagnostics and decision making since many of these discussions have taken place previously in artificial intelligence research and medical diagnostics research [112], [113]. Moreover, today we see more and more adopting of probabilistic theories not only in building systems research [82], [114]-[116], but also many other engineering disciplines [102], [117]-[121].

Quantitative modelling is used to represent the casual relationships between faults, symptoms, and impacts. Here a brief introduction is provided for readers not familiar with this topic. For a 
more detailed exploration, the classic thesis on this topic by Forbus [47] can be referenced. If graphical representation is used instead, the thesis by Daigle [122] can be referenced.

Since faults are described as discrete states such as fault-free and faulty; and the physical symptoms are usually connected to a continuous variable such as temperature and pressure, qualitative influence is used to describe their relationships:

$$
I+(S, F)
$$

$I$ denotes the there exists an influence between $S$ and $F$, and the + sign indicates positive direction of this influence. To further illustrate, this means symptom $S$ is positively influenced by fault $F$, i.e. a more positive fault $F$ makes symptom $S$ increase in the positive direction. Conversely, negative influence and undetermined influence can be presented by the following equations respectively:

$$
\begin{aligned}
& I-(S, F) \\
& I \pm(S, F)
\end{aligned}
$$

Since in this research the causal relationships between the faults and symptoms are developed based on expert knowledge and previous research, undetermined casual relationships $I \pm(S, F)$ would become uncommon. However, symptoms produced by undetermined causes might still occur, and this denotation once established, can help the operators to manually isolate fault causes. 


\subsection{Fault Detection Agent}

Both the conventional limit-checking and more advanced model-based methods can be used for detecting faults and generating symptoms. Fault symptoms generated by fault detection agents need to convey physical meanings for the other two agents to operate. Thus, some arithmetic parameters from pure black-box models may not be used for fault detection. In this thesis, greybox models is used for parameter estimation and state comparison.

The symptoms are divided into two main categories: direct-observable and indirectly estimated. Direct-observable symptoms are generated from state observers using signal models or parity equations such as conventional limit checking [50], [51] and more advanced black-box models [82], [87]. Examples of direct-observable symptoms include air temperature, supply air pressure, and so forth. Indirectly estimated symptoms are generated from physical parameters or estimated states such as infiltration rate and virtual meters. Parameters that are not directly observable are extracted from physics-based models such as R-C models [63] and autoregressive exogenous (ARX) models [84]. In general, indirectly estimated symptoms are less reliable than direct-observable symptoms for fault diagnostics and fault evaluation. Thus, uncertainty analysis needs to be applied when using indirectly estimated symptoms for fault evaluation.

\subsubsection{Parameter estimation using constrained dual Extended Kalman Filter}

As mentioned previously, some symptoms, such as low indoor air temperature readings, are directly observable from the BAS. However, many symptoms related to physical parameters that 
are useful for diagnostics, such as a room's heating/cooling sensitivity to the outdoor air temperature, are not directly observable from building's sensor network.

To tackle this issue, physical parameters from first principle models or surrogate parameters from grey-box models can be estimated using parameter estimation techniques. Surrogate parameters are related to the actual physical parameters derived from first principles, and can be used as indicators of actual parameter changes. These parameters are essential to provide an extra layer of information about building systems' behaviour to both AFDD programs and human operators.

Parameter estimation, sometimes called inverse modelling, uses sensor readings as inputs and outputs of a model to estimate its parameters. There are two types of approaches to this task: recursive estimation and sequential estimation. Recursive estimation methods collect a batch of sufficiently large data and rely on optimization to obtain the best guesses for the parameters; while in sequential methods the parameters are updated every time a new measurement is made available. Wen et al. [45] proposed using recursive least square (RLS) to obtain parameter estimates for a zone VAV system with seven parameters. Another example of recursive estimation is the genetic algorithm used by Wang and $\mathrm{Xu}[123]$.

To perform sequential parameter updates, filter-based methods are often used. Radecki et al. [63] demonstrated using Unscented Kalman Filter (UKF) to estimate thermal response parameters of an office building with a 2-node zone thermal model. Several other publications [124][69] have also demonstrated the use of extended Kalman Filter (EKF) for surrogate parameter estiamtions. In FDD applications, filter-based sequential update methods are often preferred, since they allow a faster response to system faults. In addition to parameter estimation, filters are also capable of predicting future system response, making them invaluable to model predictive control 
applications. As such, non-linear filters are often used for demand response and load shifting research [125]. Similar parameter estimation applications using nonlinear Kalman filters have been seen in many other engineering fields, such as vehicle control [126], aerospace engineering [127][128] and robotics [129].

In this thesis, a sequential parameter estimation algorithm called constrained dual Extended Kalman Filter (constrained dual EKF) was developed. This algorithm is generalized and can be combined with other building models for other building systems such as AHU, VAV terminal, etc. The proposed algorithm is aimed at improving flexibility, numerical stability and computation speed over the existing sequential methods for online MPC and FDD applications. The structure of the dual filter enables one filter (state filter) to perform state prediction such as predicting room temperature, supply air $\mathrm{CO}_{2}$ level; and another filter (parameter filter) to perform parameter estimation. While conventionally in building MPC and FDD research, joint estimation of state and parameter is often used [63], [64], [68], [69], [130]-[132]. In joint estimation, the states and parameters are estimated in a combined vector [63], which requires states and parameters to be optimized simultaneously. In dual filters, the state and parameter filters work in an alternate order and can be tweaked individually. Technically, dual estimation is harder to implement than joint estimation but it makes each filter capable of being tweaked and turned on/off individually. In addition, dual estimation has the advantage of applying different error functions to each filter as well as better convergence potential and algorithm stability. It is also capable of having one portion (parameter or state estimation) turned off to save computation time once desired results have obtained. This dual filters method, or dual estimation method, has been previously used in other research fields such as sound recognition and automobile control [126], [127], [133], [134]. 
Table 3-1 shows the generalized algorithm for constrained dual filters. Any variations of nonlinear Kalman filter can be used as the state filter to perform state predictions. Initialization of the dual filters can be achieved through optimization of model parameters with a small dataset or by applying a swarm of pre-generated dual filter particles [134]. In this thesis both filters are EKF, while other nonlinear filters such as Unscented Kalman Filters (UKF) can be used as well.

Table 3-1 Overall algorithm of Constrained dual filter

\begin{tabular}{|c|c|}
\hline 1: & Initialize dual filters \\
\hline 2: & Predict the future state vector $\hat{x}_{k}^{-}$and state covariance matrix $P_{x_{k}}^{-}$ \\
\hline 3: & Predict the future parameter vector $\widehat{\Phi}_{k}^{-}$and parameter covariance matrix $P_{\Phi_{k}}^{-}$ \\
\hline 4: & Wait until measurement vector $y_{k}$ is received \\
\hline 5: & $\begin{array}{l}\text { Calculate residual } y_{k}-h\left(\hat{x}_{k}^{-}, w\right) \text {, then update the estimated state vector } \hat{x}_{k} \text { and } \\
\text { state covariance matrix } P_{x_{k}}\end{array}$ \\
\hline 6: & If the residual $y_{k}-h\left(\hat{x}_{k}^{-}, w\right)$ is larger than the update threshold $\theta_{k}$ then: \\
\hline 7: & Update the estimated parameter vector $\widehat{\Phi}_{k}$ and covariance matrix $P_{\Phi_{k}}$ \\
\hline 8: & $\begin{array}{l}\text { If the estimated parameter vector does not satisfy the inequality constraints: } \\
D \widehat{\Phi}_{k} \leq d_{k} \& \& \widehat{\Phi}_{k} \geq 0 \text { then: }\end{array}$ \\
\hline 9: & $\begin{array}{l}\text { Perform the optimization procedure } \\
\widetilde{\Phi}_{k}=\operatorname{argmin}\left(\Phi_{k}-\widehat{\Phi}_{k}\right)^{T}\left(P_{\Phi, k}\right)^{-1}\left(\Phi_{k}-\widehat{\Phi}_{k}\right) \\
\text { subject to } D \Phi_{k} \leq d_{k} \text { and } \Phi_{k} \geq 0\end{array}$ \\
\hline 10: & Go to step 2 \\
\hline
\end{tabular}




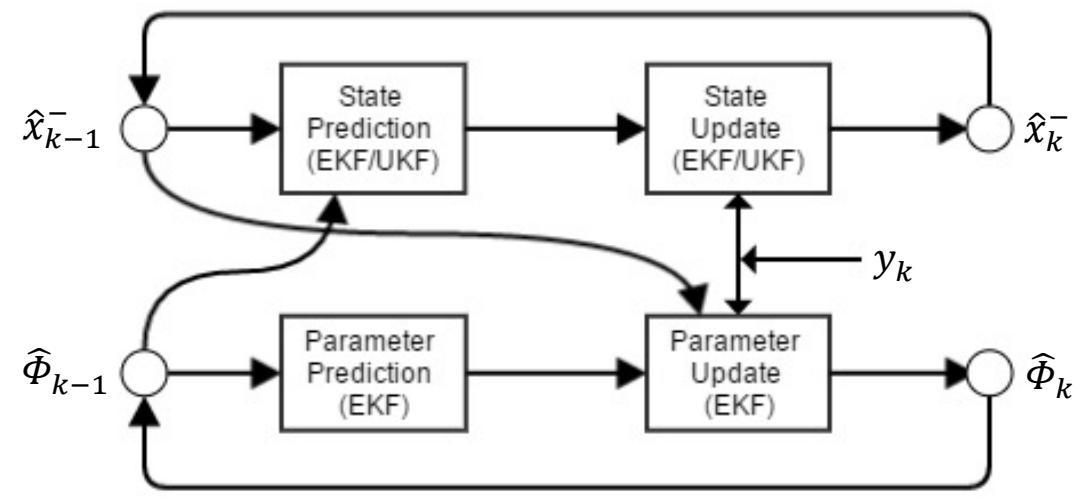

Figure 3-3 Overview of dual estimation with EKF/UKF. Each estimator can be tweaked and toggled on/off based on certain criteria

Unlike UKF, EKF have fewer tweaking parameters, making it more adaptable to dynamics of different thermal zone characteristics. The shortcoming of the EKF is that it only achieves a firstorder linearization. If the model function is highly nonlinear, UKF could provide a third order nonlinear transformation [135], [136].

The dual estimation method works in an alternating manner [137], as shown in Figure 3-3. State and parameter estimation from the last time step is used for state prediction, and state estimation from the last time step is used in the parameter update step. Measurement of the actual system -$y_{k}$, is used for updating both filters.

The model functions for the state filter and parameter filter in dual estimation are:

$$
\text { State filter: } \quad \text { Parameter filter: }
$$

$$
\widehat{x}_{k}^{-}=f\left(\hat{x}_{k-1}, \widehat{\Phi}_{k-1}, u_{k-1}, w_{s}\right) \quad(4) \quad \widehat{\Phi}_{k}^{-}=\widehat{\Phi}_{k-1}
$$

where $k$ denotes time step, $\hat{x}$ is the estimated state vector and $\widehat{\Phi}$ is the estimated parameter vector, $\hat{x}^{-}$and $\widehat{\Phi}^{-}$are the predicted state vector and the parameter vector, and $w_{s}$ is the process noise. 
After the definition of model functions, the prediction step of the constrained dual EKF becomes:

\section{Prediction Step}

\section{State filter:}

$$
\begin{aligned}
& \widehat{x}_{k}^{-}=f\left(\widehat{x}_{k-1}, u_{k-1}, w_{s}\right) \\
& P_{x_{k}}^{-}=F_{k} P_{x_{k-1}} F_{k}^{T}+R^{v}
\end{aligned}
$$

\section{Parameter filter:}

$$
\begin{gathered}
\widehat{\Phi}_{k}^{-}=\widehat{\Phi}_{k-1} \\
P_{\Phi_{k}}^{-}=\lambda^{-1} P_{\Phi_{k-1}}
\end{gathered}
$$

Forget factor: $\lambda \in(0,1]$, Jacobian matrix of $f: F_{k}=\left.\frac{\alpha f\left(\widehat{x}_{k-1}, u_{k-1}, w_{s}\right)}{\alpha x}\right|_{x=\widehat{x}_{k-1}}$

In this prediction step, a forget factor $\lambda$ is introduced when calculating the covariance matrix for the parameter filter. The forget factor dictates how sensitive the parameter filter is to the latest observation with a range of between 0 and 1 . A value closer to 1 makes the parameter filter less sensitive to latest measurements, and vice versa. A low forget factor value may cause the filter to become too sensitive and more likely to become unstable due to measurement noises and model errors. The forget factor is closely related to the time step the filters use, a filter with more frequent updates and shorter time step tends to benefit from a forget factor closer to 1 than a filter with longer time step. $R^{v}$ is the process noise covariance, which is used to represent uncertainties caused by modelling error. 
After the state vector is predicted and the actual observations of the predicted states are collected, the update step can be performed to adjust the state filter and parameter filter to reflect the actual measurements. The update step of dual EKF is:

\section{Update Step}

\section{State filter:}

$$
\begin{gathered}
\mathbf{K}_{k}^{x}=\boldsymbol{P}_{x_{k}}^{-} H_{k}^{T}\left(H_{k} P_{x_{k}}^{-} H_{k}^{T}+R^{n}\right)^{-1} \\
\widehat{x}_{k}=\widehat{x}_{k}^{-}+\mathbf{K}_{k}^{x}\left[y_{k}-h\left(\widehat{x}_{k}^{-}, w\right)\right] \\
P_{x_{k}}=\left(I-K_{k}^{x} H_{k}\right) P_{x_{k}}^{-}
\end{gathered}
$$

\section{Parameter filter:}

$$
\begin{gathered}
K_{k}^{\Phi}=P_{\Phi_{k}}^{-}\left(H_{k}^{w}\right)^{T}\left[H_{k}^{w} P_{\Phi_{k}}^{-}\left(H_{k}^{w}\right)^{T}+R^{e}\right]^{-1} \\
\widehat{\Phi}_{k}=\widehat{\Phi}_{k}^{-}+K_{k}^{\Phi} e_{k}
\end{gathered}
$$

$\mathrm{K}_{k}^{x}$ and $K_{k}^{\Phi}$ are Kalman gains for the state filter and parameter filter respectively. Kalman gain dictates how much the state and parameters vector need to be adjusted given the same residuals. $R^{n}$ and $R^{e}$ are the measurement noise covariance for the state and parameter filter respectively. If constant diagonal matrices are used for these two covariances, the effect of these two covariances will be cancelled out [134].

The selection of $H_{k}^{w}$ and $e_{k}$ depends on the cost function. In this application, the maximumlikelihood cost function is used [129]:

$$
\begin{aligned}
& H_{k}^{w}=\left.\frac{\alpha h\left(\hat{x}_{k}^{-}, w\right)}{\alpha \Phi}\right|_{\Phi=\widehat{\Phi}_{k}^{-}} \\
& e_{k}=y_{k}-h\left(\hat{x}_{k}^{-}, w\right)
\end{aligned}
$$

Other cost functions can be used for different applications [137]. For example, expectationmaximization (EM) in which the cost function decreases over iterations, can be used when only parity equation is needed after the training period. 
Many times due to measurement noise, system non-linearity, and inappropriate initial conditions, both EKF and UKF can diverge and become numerically unstable [138]. An example of such instabilities in EKF is given in Figure 3-4: the parameter experiences negative tracking divergence thus making the model to become unstable. To keep the parameters within a stable range, inequality constraints and optimization can be applied [138][139]. After each parameter filter update, an inequality check is performed:

$$
D \Phi_{k} \leq d_{k} \text { and } \Phi_{k} \geq 0
$$

where $D$ is a matrix determining which parameters have inequality constraints, and $d_{k}$ is the constraint vector. If the result does not satisfy inequality constraints, then an optimization procedure is followed:

$$
\begin{gathered}
\widetilde{\Phi}_{k}=\operatorname{argmin}\left(\Phi_{k}-\widehat{\Phi}_{k}\right)^{T}\left(P_{\Phi, k}\right)^{-1}\left(\Phi_{k}-\widehat{\Phi}_{k}\right) \\
\text { subject to } D \Phi_{k} \leq d_{k} \text { and } \Phi_{k} \geq 0
\end{gathered}
$$

Since the covariance matrix of the parameters $P_{\phi, k}$ is positive definitive, this optimization task is strictly convex can be solved with simple methods such as gradient-base methods. The inequality constraints can be turned off for different applications, but it is not recommended.

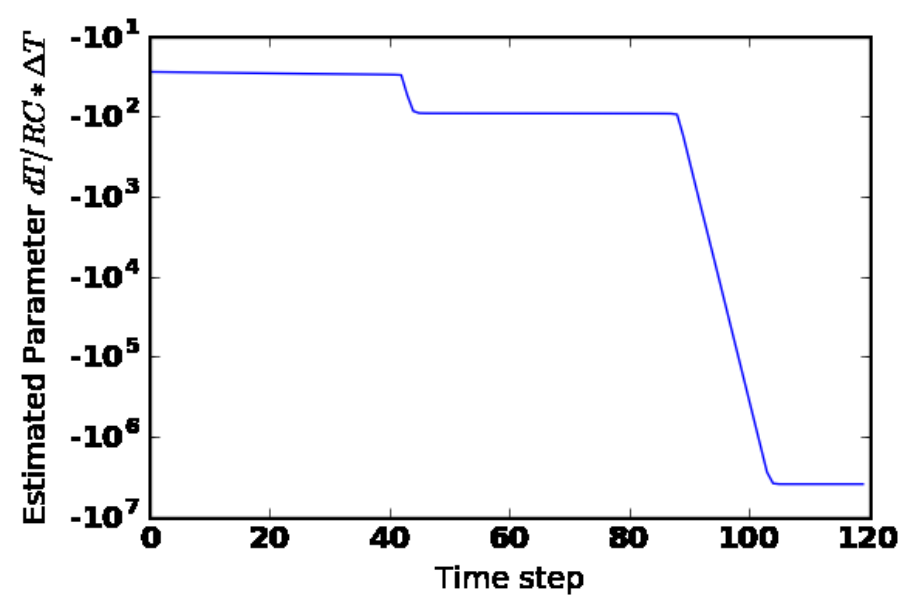

Figure 3-4 Example of a diverged parameter causing the reduced model to become unstable 
A custom class of constrained dual EKF is written by the author in Python. The author used SymPy [140] to handle symbolic mathematics and calculate Jacobian matrices for the model functions. For parameter optimization when inequality constraints are violated, the author applied the Constrained Optimization BY Linear Approximation (COBYLA) [141] adaption in Scipy [142]. This custom class can also be used without inequality constraints as a regular dual EKF. For other regular nonlinear filers, libraries such as FileterPy [143] and pykalman [144] can be used instead. The author can be contacted for the constrained dual EKF code. The author could also provide its implementation in zone-level parameter estimation, model initiation and its UKF variation.

To investigate the effectiveness of the proposed parameter estimation method, a parametric analysis is performed using a shoebox EnergyPlus model. The proposed parameter estimation process is expected to detect parameter variations quantitatively. Figure 3-5 shows the results of the parametric analysis. Overall the parameter estimation technique could detect weakly nonlinear thermal characteristics such as infiltration and equipment load heat gains. However, it is harder to detect highly nonlinear thermal processes such as conduction heat transfer through the envelope, probably due to the R-C model employed. This parameter estimation method and these results are currently in the process of being published, an case study for fault detection was also published in a previous study [69]. 

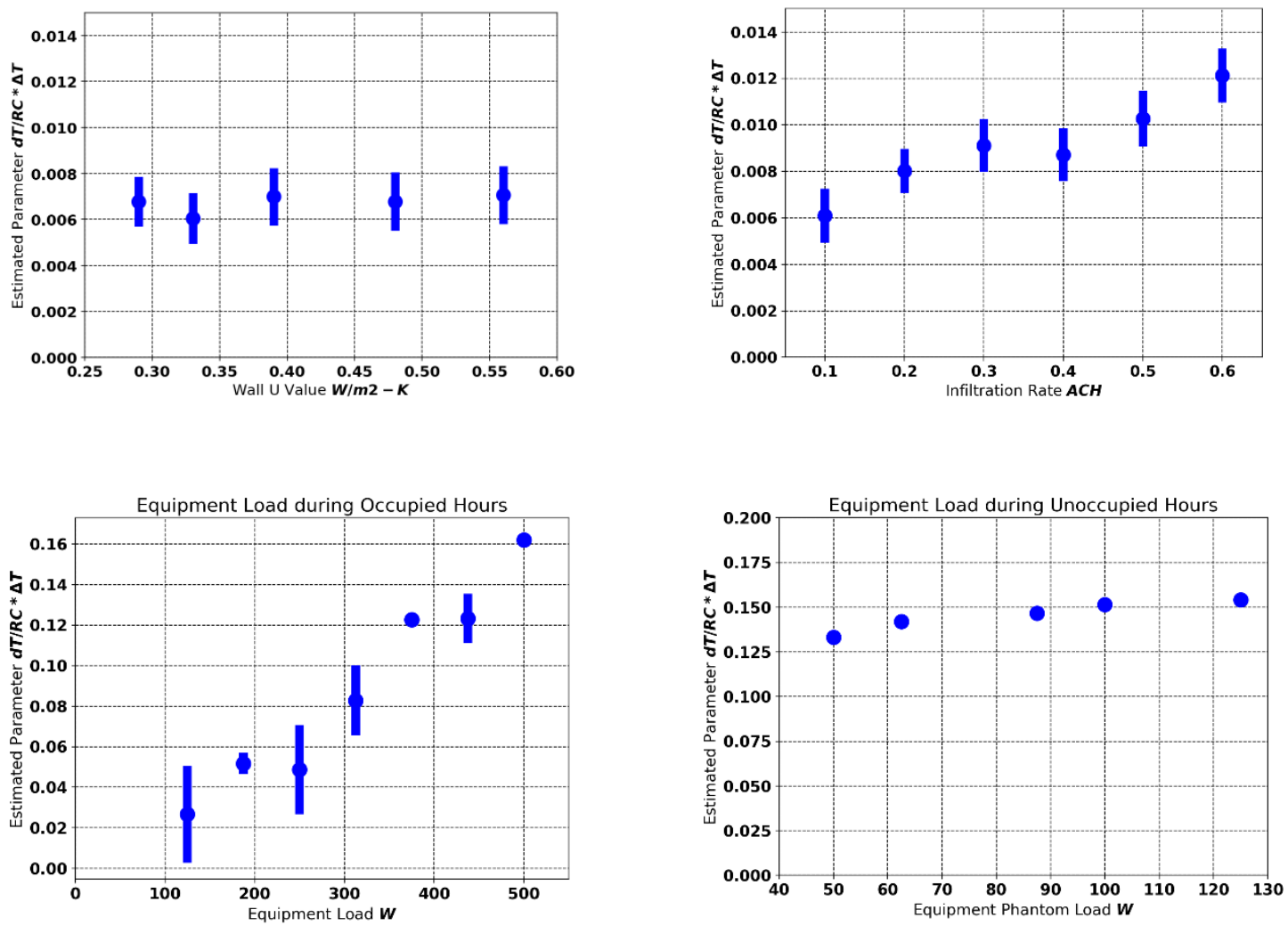

Figure 3-5 Sensitivity analysis for parameter estimation. From a) to d): wall thermal resistance, zone infiltration, equipment loads during occupied hours, constant equipment load. One standard deviation is used as the error bar.

\subsubsection{Probabilistic symptom generation}

This section introduces the procedures of quantifying symptom severity and use it to estimate the probability of a positive symptom. As identified in the previous section, there are two types of symptoms to be quantified -- directly observed and indirectly estimated. Inherently indirectly observed symptoms possess more uncertainties from their counterparts. However, this does not mean directly observed symptoms are automatically reliable; noise caused by sensor errors and measurement errors could also make these observations less than perfect. 
Table 3-2 Examples of symptom description

\begin{tabular}{cccc}
\hline Equipment & Symptom & Description & Direct Observation \\
\hline Supply Fan & $\boldsymbol{S}_{\boldsymbol{s} \mathbf{1}}$ & Fan efficiency & No \\
\cline { 2 - 4 } & $\boldsymbol{S}_{\boldsymbol{s} \mathbf{2}}$ & Fan speed & Yes \\
\hline \multirow{2}{*}{ AHU } & $\boldsymbol{S}_{\boldsymbol{s a 1}}$ & Supply air flow rate & Yes \\
\cline { 2 - 4 } & $\boldsymbol{S}_{\boldsymbol{o a} \mathbf{1}}$ & Outdoor air flow rate & Yes \\
\cline { 2 - 4 } & $\boldsymbol{S}_{\boldsymbol{v} \boldsymbol{h} \mathbf{1}}$ & Reheat fluid flow rate & Maybe \\
\hline Zone Level & $\boldsymbol{S}_{\mathbf{z h} \mathbf{1}}$ & Heating sensitivity & No \\
\cline { 2 - 4 } & $\boldsymbol{S}_{\mathbf{z t} \mathbf{1}}$ & Air temperature & Yes \\
\cline { 2 - 4 } & $\boldsymbol{S}_{\mathbf{z i 1}}$ & Zone infiltration & No \\
\hline
\end{tabular}

The observability of the symptoms will affect how the faults will be evaluated inside the fault evaluation agent. Table 3-2 shows examples of symptom descriptions and if they are directly observable. A complete list of symptoms studied in this research is provided in the next Chapter. In this framework, the severity of a symptom is denoted by $\mathrm{z}$ and is calculated using two different methods: limit checking and z-score. Limit checking is applied for direct observable symptoms since normal range of operation can be established for most of these symptoms. For more complicated symptoms in which the normal operation range cannot be established, such as estimated parameters and heating sensitivity, z-score can be calculated by establishing a training benchmark.

To perform limit checking on a certain value such as air temperature or rate of temperature change, severity score of the symptom can be calculated as:

$$
z= \begin{cases}\frac{2\left(x-x_{\max }\right)}{x_{\max }-x_{\min }} & \text { if } x>x_{\max } \\ \frac{2\left(x-x_{\min }\right)}{x_{\max }-x_{\min }} & \text { if } x<x_{\min }\end{cases}
$$


where $x_{\max }$ and $x_{\min }$ are the limit thresholds of the value. Not only static values, but also dynamic limits from some fault detection methods can be used to establish the limits. Dynamic limits include parity functions that predicts supply air temperature [145] or electricity usage [80], as long as those severity values infer some physical relationships and which can be used in the fault diagnostics process.

For estimated parameters, severity can be calculated by using the z-score, assuming those parameters are normally distributed [146]:

$$
\mathrm{z}=\frac{\mathrm{x}-\mu}{\sigma}
$$

Where $\mu$ is the mean and $\sigma$ is the standard deviation of the variable. The mean and standard deviation of the value can be trained using a set of data during commissioned operation. Methods such as weighted moving average or filters could also be applied to sequentially or periodically update the mean and standard deviation.

After the severity scores are calculated, logistic regression can be used to obtain the probability of a symptom state $p(s)$. The logistic curve can be trained with fault-free data to lower false positive rates. The fault-free data can be obtained during the commissioning.

$$
\mathrm{p}(\mathrm{s})=\frac{1}{1+\mathrm{e}^{-\mathrm{k}\left(|\mathrm{z}|-\mathrm{z}_{0}\right)}}
$$

Where $\mathrm{k}$ and $\mathrm{z}_{0}$ are tweaking parameters for the logistic regression and can be trained given available data. An example of different shapes of the logistic regression is shown in Figure 3-6. It is worth noting that positive and negative $z$ values may indicate different symptoms states (e.g., room temperature too low is a negative value). A negative severity value needs to be used with a different logistic function than a positive value. Another characteristics of the symptom in this framework is the capability of using ordinal descriptions such as Normal/Minor/Major with 
different logistic regression curves, instead of using the conventional Bernoulli distribution of Normal/Faulty.
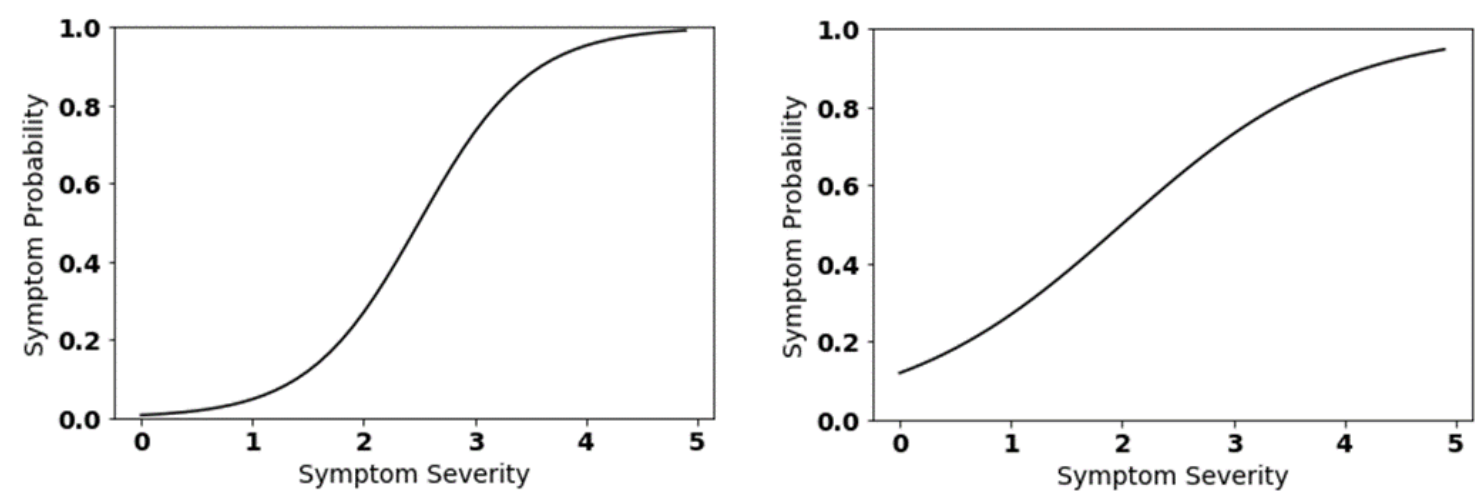

Figure 3-6 Example of different logistic regression curves for calculating $p(s): z 0$ is the position of $z$ score where symptom starts occurring, $k$ is the slope of the logistic regression (confidence of symptom over the threshold)

To make this symptom generation process easier to implement, three levels of logistic regression curves are established as templates. Values of $\mathrm{k}$ and $\mathrm{z}_{0}$ are defined as in Table 3-3. Lower $\mathrm{k}$ and $\mathrm{z}_{0}$ values indicate a symptom is more likely to be true with smaller deviations, while higher $\mathrm{k}$ and $\mathrm{z}_{0}$ values mean only a high deviation from the normal range can make the symptom to be true. On the other hand, $k$ and $z_{0}$ values too low can make the symptom too sensitive to its inputs, causing higher false positive rate, thus causing false positive fault diagnosis; while values too high can also make the symptom to insensitive to its inputs, leading to higher false negative rate, eventually making the fault diagnostics symptom fail to perform its tasks. 
Table 3-3 Templates for logistic regression

Logistic Curve Template $\quad \boldsymbol{k} \quad \mathbf{z}_{\mathbf{0}}$

\begin{tabular}{r|cc}
\hline Low & 1.5 & 1 \\
Medium & 3 & 3 \\
High & 6 & 5
\end{tabular}

Figure 3-7 shows probabilities for increased infiltration rate symptom, calculated from using two different logistic regression curves during an unintended window opening fault. As discussed earlier, when appropriate $k$ and $z_{0}$ are used, the symptom probability can be calculated relatively reliably. Still, in this research, the selection of the logistic regression curve for each symptom is a manual process. To automate this in the future, optimization of $k$ and $z_{0}$ can be applied for each symptom to make probability calculations sensitive enough to the actual evidence, while minimizing the risk of false positives. This automated calibration process will be part of the future work covered in the discussion section. 

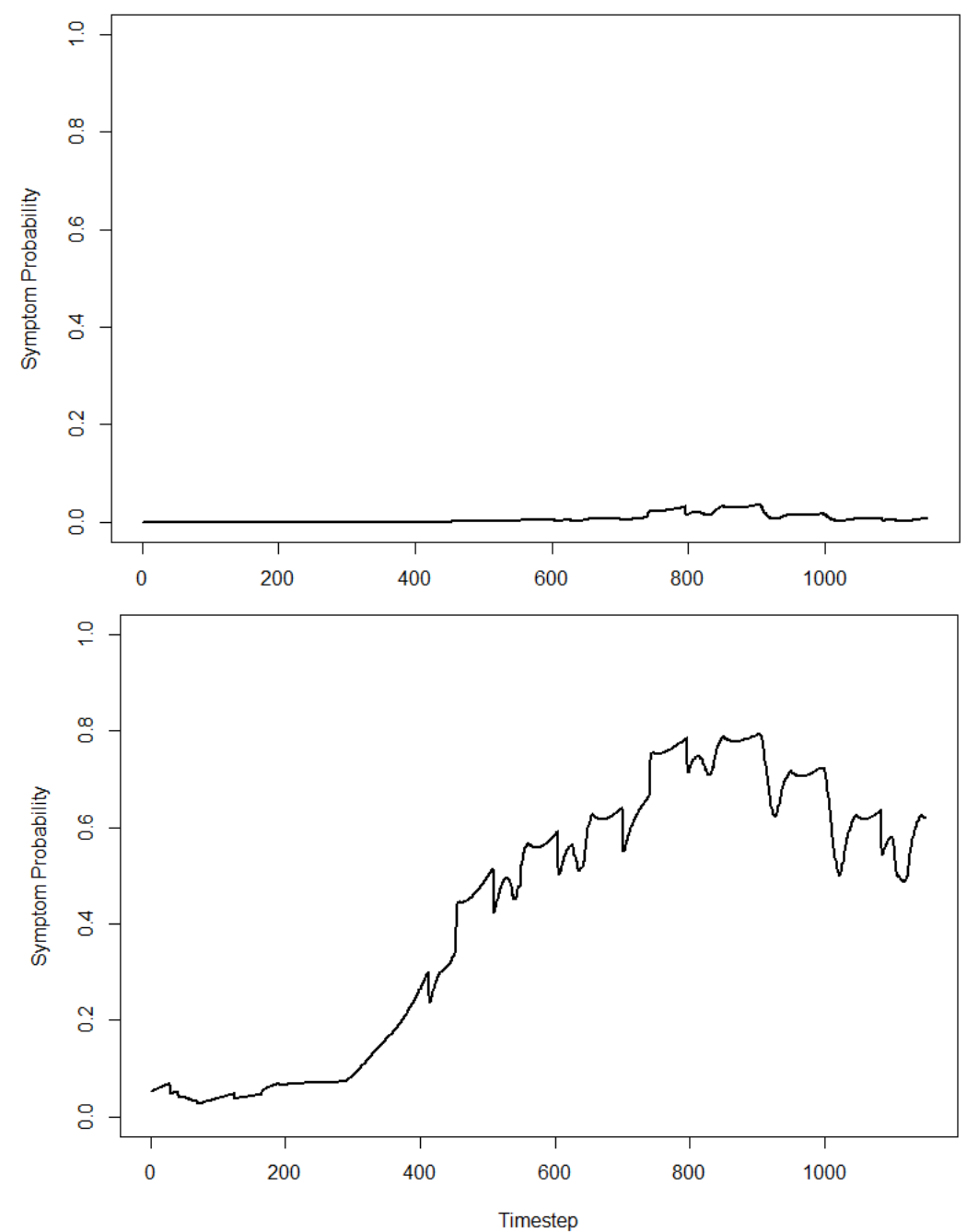

Figure 3-7 Example of a symptom probability calculated from different logistic regression templates. Above: calculated from the Medium template; Below: calculated from the Low template

\subsection{Fault Diagnostic Agent}

The fault diagnostic agent runs at a predefined time step, and recursively obtains symptom distributions from the fault detection agents to update fault belief. The selection of diagnosis intervals (time step) depends on whether the faults are time sensitive. For time-critical systems such as central air handling units and sizeable mechanical equipment, the fault diagnostics agent can run at a small time step in the order of seconds or a few minutes. For slow response systems 
such as rooms and thermal zones, the fault diagnostic agent can run at a time step of minutes or even hours.

Fundamentally the fault diagnostic process is a classification problem - classify the status of all faults of interest into different states (normal, minor, True/False, etc.). This process can be achieved by the heuristic reasoning of a human, or decision models inside a computer. Since this thesis is focused on automated FDD, only computer-based method will be discussed. Although other fault diagnostics methods such as decision trees or heuristics can be used in this agent, Dynamic Bayesian Network (DBN) is chosen based on literature review. DBN is a specialized Bayesian Network (BN) with the following characteristic:

1. BN can be constructed from causal relationships between faults and symptoms, making it easier to construct than commonly used expert systems such as decision trees [147]. In addition, unlike decision trees, causal relationships contained within the $\mathrm{BN}$ are physicsbased and can be ported between different buildings.

2. The structure of the network can use quantitative symptoms as well as qualitative observations as evidence. Moreover, if the observations are not available, BN can dynamically adjust its structure to perform diagnosis. It can also provide guidance in terms of which additional evidence is needed. For example, if multiple faults are diagnosed as a possible cause for low indoor temperature, the occupant or a building operator can provide additional observation(s) to the $\mathrm{BN}$ so that unrelated diagnoses can be eliminated.

3. BN can work in both directions. Besides evidential reasoning (inference) used for fault diagnostics, BN can also be used for causal reasoning. This means that if a fault has been diagnosed, it is also possible to locate possible symptoms that are not directly observable. 
4. BN can also be used to include the effects of fault propagation and avoid false positives downstream. For example, if the central AHU has been diagnosed with stuck heating valve making the supply air temperature too low, the under-heated zones will not be diagnosed with reheat valve issues.

DBN is a specialized BN for continuous systems. Similar to a Markov Chain, DBN carries over past information for future decision-making. This means evidence can accumulate over time to detect minor or drifting faults, and false positives can be cancelled out by normal conditions.

\subsubsection{Dynamic Bayesian Network}

Bayesian Network (BN), or Bayesian Belief Network, is one of the most effective classifiers used in artificial intelligence research [148]. BN has been widely researched as a decisionmaking tool in fields such as medical diagnosis, risk management, space exploration, aerospace, and much more [149]-[153]. BN has proven its effectiveness in multiple building AFDD applications [76], [114], [147], [154], [155], as well as in other fields such as medical diagnostics [149], [156] and reliability engineering [121], [157]. A Bayesian Network is an acyclic graphical model for probabilistic relationships among random variables [148], as seen in Figure 3-8. While many other fault diagnosis systems such as fuzzy logic and heuristic rules emulate an expert's reasoning process, $\mathrm{BN}$ directly represents the causal relationships with uncertainties between faults and symptoms as well as fault propagations (one fault leading to another). The main advantage of $\mathrm{BN}$ is its ability to establish causal relationships from expert knowledge, i.e. prior knowledge. Another advantage is its ability to update its parameters to increase its accuracy over time [148], [158], [159]. It is suitable for classification problems where the causal relationships between the variables are known and can be predefined manually. In cases where the causal 
relationships are unknown or cannot be defined manually, BN can use structure learning to determine those relationships. However, by using structure learning, BN loses its advantage of incorporating prior knowledge and becomes one of the many other classifiers available from machine learning. When the causal relationships between the variables become cyclic, such as a fault leading to a symptom which aggravates the same fault, then BN cannot be applied.
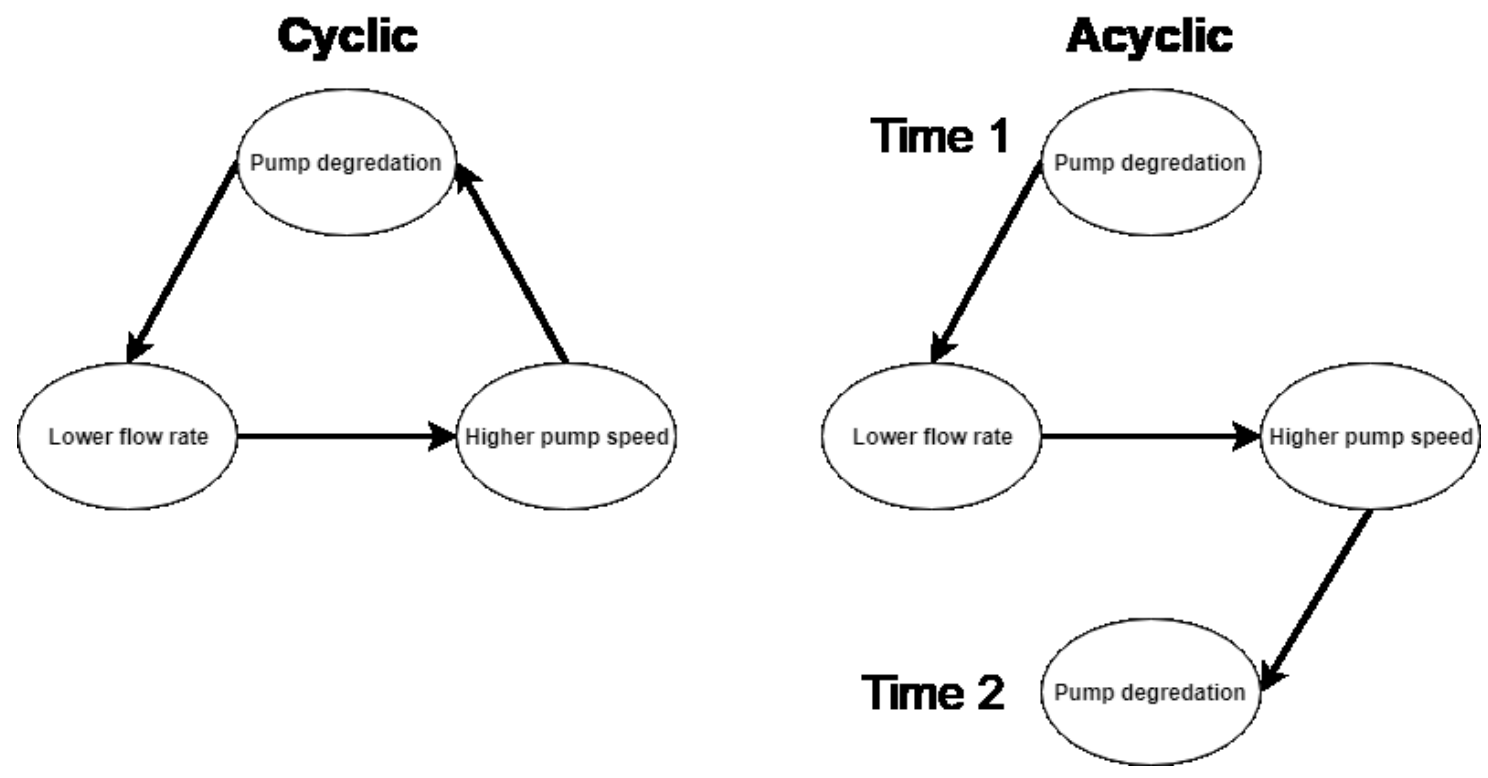

Figure 3-8 Cyclic and acyclic graphical probabilistic models

Most of the BNs employed in engineering system FDD are two layer networks (symptom-faults) [99], while in medical diagnosis applications the network is more complex and external facts such as age and gender are part of the diagnosis. Also, most of the faults inside engineering systems are affected by externalities such as time since last maintenance; those factors should be included in the DBN as well.

In this thesis, $F_{1}, \ldots, F_{n}$ denote faults, while lower case $f_{1}, \ldots, f_{i}$ are the fault states. Similarity, $S_{1}, \ldots, S_{m}$ are symptoms related to the faults, or children; while the lower case $s_{1}, \ldots, s_{k}$ are the symptom states, as discussed earlier. Note that the symptom values in this work are 
graded/ordinal due to the usage of the leaky noisy-max model, which will be further explained in the following section.

To construct the BN, prior probability of fault need to be defined, which is denoted by $P(F)$. The prior probability represents the natural probability of a fault happing during a time step. In this application, it is assumed $99.9 \%$ of the time the fault would be fault-free, and the rest of the $0.1 \%$ probability is equally distributed to the rest of the fault states. For example, for a fault with two states (normal, faulty), its prior probability would be $(0.999,0.001)$; for a fault with three states (normal, negative stuck, positive stuck), its prior probability would be $(0.999,0.0005,0.0005)$, respectively.

In addition to prior probability, $P(S \mid F)$-- the conditional probabilities between faults and symptoms also need to be defined. Conditional probability, sometimes called likelihood, represents the probability of symptom states is based on different fault state combinations. A table called conditional probability table (CPT) is used to store the conditional probabilities of all possible fault-symptom state combinations. The creation of this CPT will be detailed in a later section.

Thus, the basic probabilistic representation of the symptom-fault relationship from Bayes' theorem is:

$$
P(F \mid S)=\frac{P(F) P(S \mid F)}{P(S)} \propto \sum_{y} P(F, S, y)
$$

where $y$ is the unobserved variable, and posterior $P(F \mid S)$ is the probability of each fault states given observed symptoms. The inclusion of the unobserved variable takes the uncertainties from the symptoms into the account. This is important since there are limitations to how many and how accurate we can observe and identify the symptoms. The posterior distribution, $P(F \mid S)$, should be treated as belief of fault states given the observation of symptoms at a given time step. 


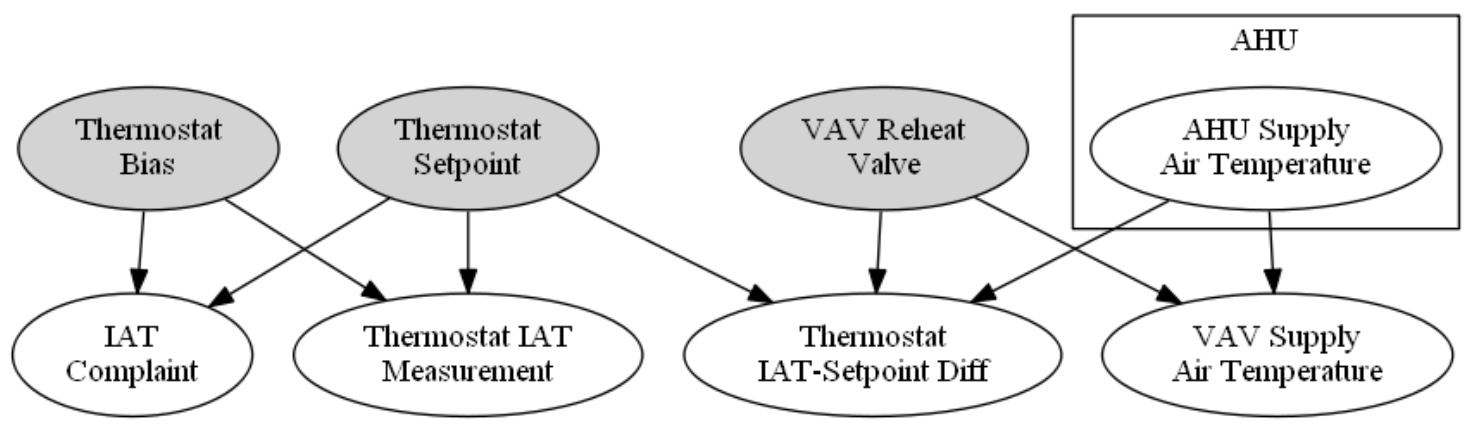

Figure 3-9 Example of a BN used for VAV level fault diagnostic, the arrows denote causal relationships between the node and starts from cause to effect; shaded nodes are faults

Figure 3-9 shows an example of how to use BN to represent causal relationships between faults and symptoms. There are several particular points about this example:

1. Externalities such as $V_{1}$ can be added to $\mathrm{BN}$ to represent factors that might affect the faults. Factors such as equipment age, data-since-last-maintenance could directly affect the probability of a fault occurring.

2. Fault propagation could also be added, as demonstrated by $P_{p}$ propagating its impact to symptom $S_{a}$. Note that due to usage of the leaky noisy-max model introduced in the later section, faults should independently affect the symptom, so fault propagation such as $F_{p}$ and $F_{a}$ shall not share a common symptom node. An example of fault propagation is shown in Figure 3-10. 


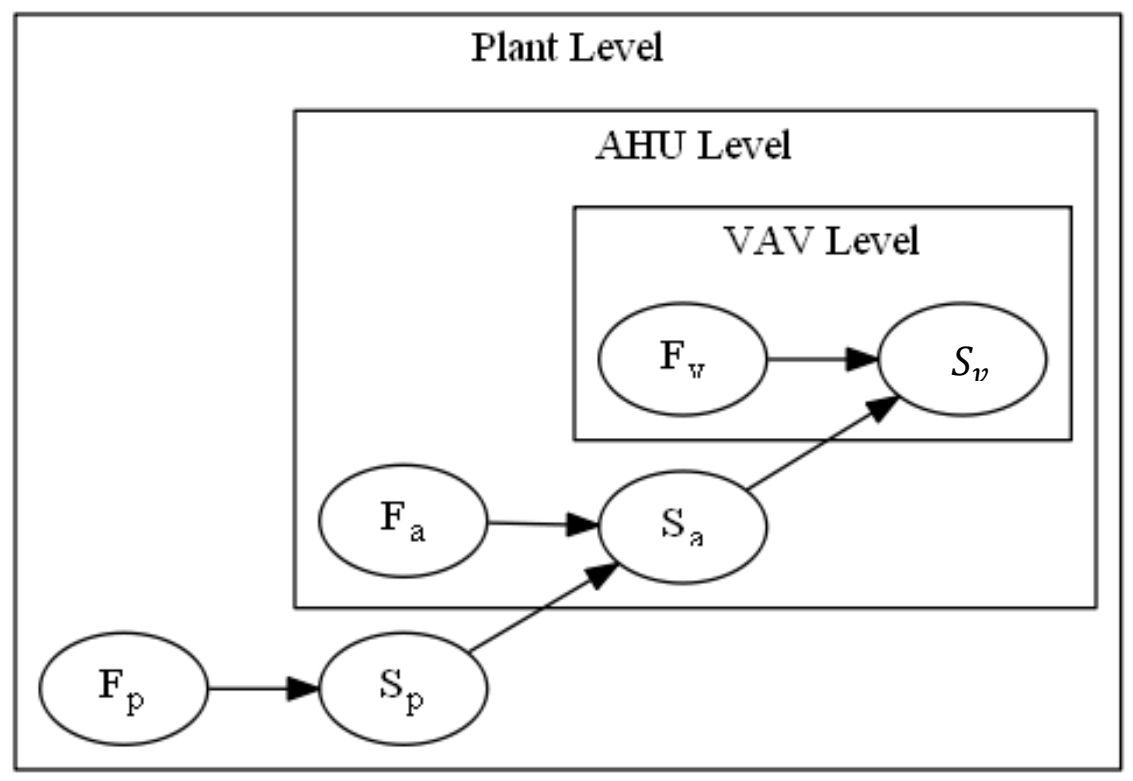

Figure 3-10 Example of fault propagation from plant level to VAV level

When used in fault diagnostics applications, the BN mentioned above acts as an event-based system. It makes the evaluations independent of their previous results and does not accumulate evidence over time. While this is fine for a diagnosis based on single-time observations such as medical diagnostics [149], [156], it may not be suitable for building systems when most of them are operating continuously. To amend this, Dynamic Bayesian Network is introduced.

One option to incorporate evidence over time is to use the Dynamic Bayesian Network (DBN). DBN, also commonly called Two-Timeslice Bayesian network (2TBN), is quite popular for temporal data produced by causal processes such as building faults-symptoms and sequence data such as natural language and bio-sequence [160]. Another advantage of DBN is its ability to segregate some cyclic relationships (Figure 3-8) into different time steps. This is particularly useful for faults with reinforced cycles. In the field of FDD and reliability, there only has been limited research using DBN for continuous systems [121], [150], [161]. A conventional BN used 
in other FDD research can be converted to a DBN by establishing CPTs between the same faults in adjacent time steps:

$$
P\left(F_{t+1}^{i} \mid F_{t}^{i}\right)
$$

And the previous BN starts to look like Figure 3-11:

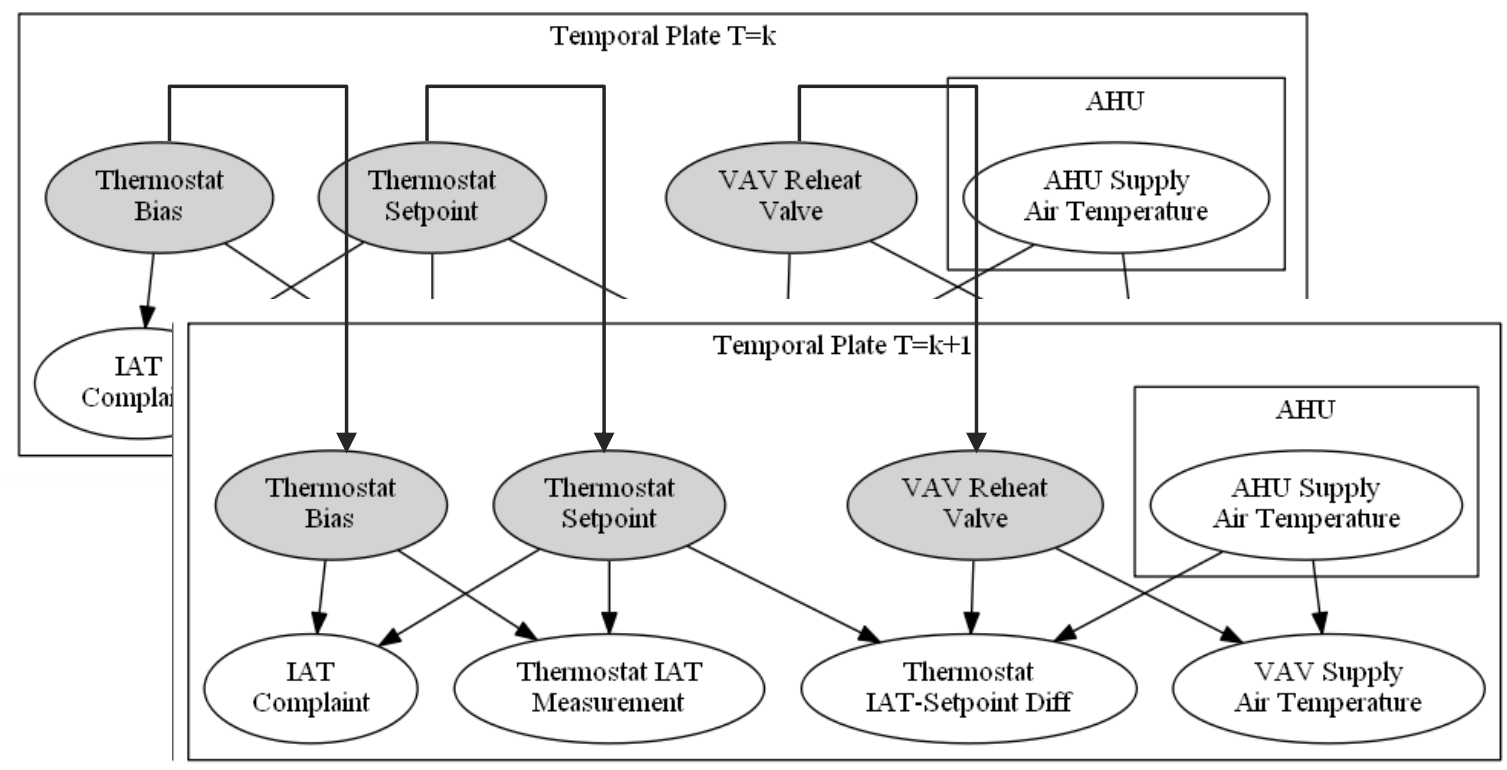

Figure 3-11 Example of the Dynamic Bayesian Network, fault probabilities from a previous time step affect the fault probabilities of the next time step

To further illustrate, the belief of a fault from a previous time step gets carried over to the next time step and its highest probable state is diminished. For an abnormal fault value of $\boldsymbol{f}$, if $p\left(\boldsymbol{f}_{t+1} \mid \boldsymbol{f}_{t}\right)=1$ then the fault belief does not diminish; on the other hand, if $p\left(\boldsymbol{f}_{t+1} \mid \boldsymbol{f}_{t}\right)<1$, then $p\left(\boldsymbol{f}_{t+1} \mid \boldsymbol{f}_{t}\right)$ can be considered similar to a forget factor in iterative algorithms, where future information becomes more important and past fault belief decays over time. In general, the inclusion of this stepwise conditional probability will make the fault diagnostic algorithm less 
sensitive to sudden errors, decreasing the overall likelihood of false positive and false negative diagnosis.

A custom DBN class is created by the author in $\mathrm{R}$ based on the gRain package [162]. The R code is is available upon request. The custom DBN class includes handling of time steps in Dynamic Bayesian Network as well as calculating the conditional probability tables based on leaky noisymax abstraction, which will be introduced in the next section. gRain uses the Lauritzen \& Spiegelhalter (LS) algorithm [163] to query its probabilistic graphical model, in this case, for inferring posterior probabilities of faults. A commercial product, RHugin, provides faster computation than gRain. Thus, for applications beyond prototyping stage, RHugin may be a better alternative than the gRain package.

The reason to use algorithms such as LS to approximate posterior distribution of $P(F \mid S)$ instead of analytically solving it with Bayes' theorem is due to the complexity of the calculation, as the analytical solution is a NP-hard problem [164]. This thesis will not go into detail about the LS algorithm, but some high-level summary is provided here.

There are many well-established algorithms used for approximating discrete Bayesian Networks such as LS algorithm, Hugin algorithm [165] and Shenoy-Shafer (SS) [166] algorithm. Lepar and Shenoy [167] performed a comparison of three different BN inference algorithms (LS, SS, and Hugin) concluded that storage space is sacrificed when trying to achieve more efficient computation. In this case, SS is faster than Hugin, and Hugin is faster than LS; while SS requires more storage space than Hugin, and Hugin requires more storage than LS. In modern computers where memory is no longer limited, Hugin and SS may be better candidates for deployment. 
For learning conditional probabilities and structural causal relationships, machine-learning related topics can be explored, and learning algorithms such as Expectation-Maximization, Metropolis-Hasting, and others can be applied.

A direct comparison of using BN and DBN for fault diagnostics is shown in Figure 3-12.

Compared to BN, which only intermittently produced high fault probabilities, DBN was able to keep fault probability high, thus providing a more consistent fault report to the users.

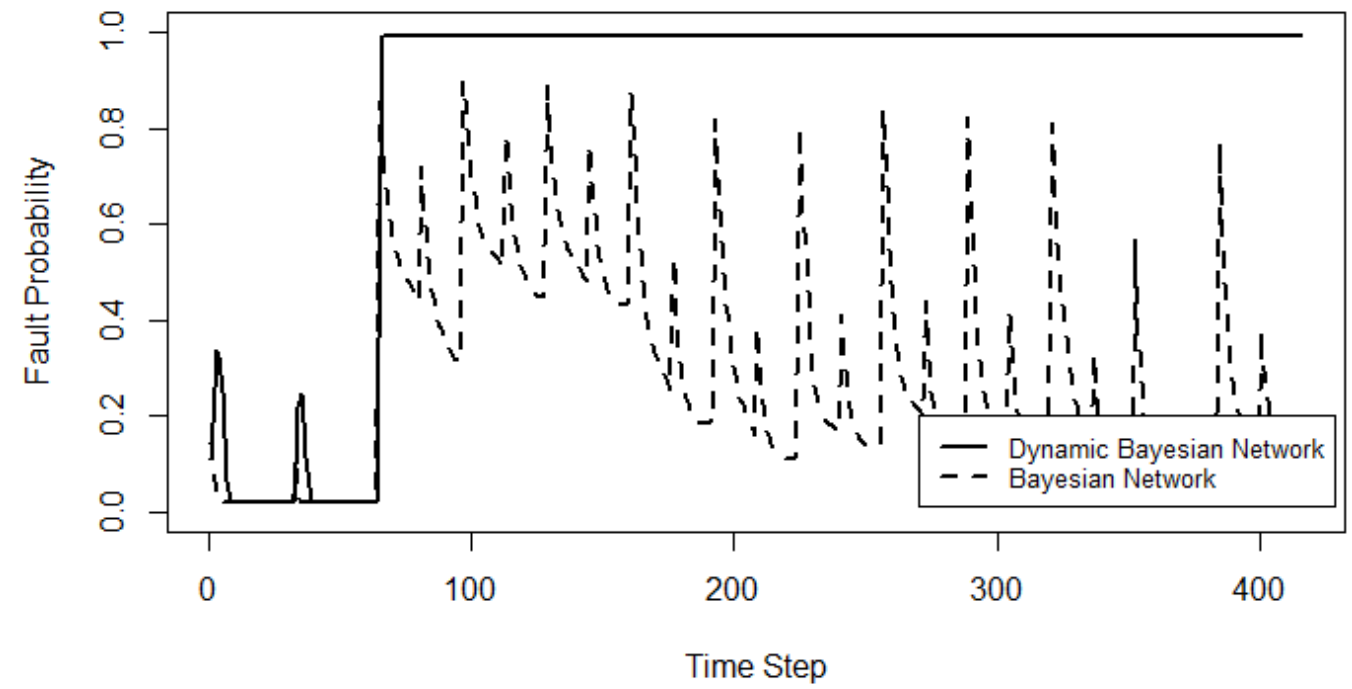

Figure 3-12 Example of fault probabilities of using BN and DBN (published in ASHRAE Transactions) [168]

\subsubsection{Leaky Noisy-Max model}

Two probabilities need to be defined inside the DBN: the prior probability of a fault happening -$P(F)$, and the conditional probability of a symptom state occurring given the fault state -$P(S \mid F)$. Then based on the observation of symptom states, $P(S), P(F \mid S)$ can be inferred. To achieve this, the conditional probability tables (CPT) between symptoms and faults need to be established. CPTs define the conditional probabilities of each fault states under every combination of the connected symptoms. For instance, for a binary fault $F_{1}$ with two related 
three-state symptoms $S_{1}, S_{2}$, a total number of 18 conditional probabilities need to be defined, such as $P\left(f_{1}^{1} \mid s_{1}^{1}, s_{2}^{1}\right), P\left(f_{1}^{1} \mid s_{1}^{1}, s_{2}^{2}\right), P\left(f_{1}^{1} \mid s_{1}^{1}, s_{2}^{3}\right), P\left(f_{1}^{1} \mid s_{1}^{2}, s_{2}^{1}\right), P\left(f_{1}^{1} \mid s_{1}^{2}, s_{2}^{2}\right) \ldots$ For $n$ fault nodes each with $i$ states, each fault with $m$ releated symptoms with $k$ symptom states, the size of the CPT becomes $n i k^{m}$.

Naturally, the definition of CPT becomes tasking when the BN becomes more complicated, especially in cases where the number of symptoms grows larger. In many complicated BN applications, manual definition of CPT would become impractical or nearly impossible. Some researchers opted to learn the CPT from data. However, since fault data is relatively scarce for building systems, the author still used a manual process to set up the CPTs for the case studies. To reduce the exponential growth in complexity of the CPT, leaky noisy-max gates [117], [169] can be used to simplify the manual definition process. Similar simplifications have been seen in medical diagnostic networks [169], [170], as well as building FDD research [98], [147], [171]. To apply the leaky noisy-max model, faults are assumed to be independent causes of symptoms as well as independent of each other [117]. Most importantly, the symptoms have to be graded/ordinal variables [117]. In other words, the symptom should take on values ordered by severity such as normal, minor, major, and severe. Non-ordinal symptoms such as Negative/Normal/Positive cannot be used in conjunction with the leaky noisy-max model due to the way the conditional probability table is abstracted by this model.

The CPT can be calculated by first defining a leak probability $c_{S}^{L}$ for the symptom when all faults are absent. Since in this application, the first fault state is assumed to be normal or fault-free, $c_{S}^{L}$ becomes:

$$
c_{\mathrm{s}}^{\mathrm{L}}=\mathrm{P}\left(\mathrm{s} \mid \mathrm{f}_{1}^{1}, \ldots, \mathrm{f}_{n}^{1}\right)
$$


Some BN abstractions do not use a leak probability term, which means when all causes are absent the effect has zero probability of happening. Due to the uncertainties when describing the causal relationships between faults and symptoms inside building systems, the author would argue the adoption of leak probabilities is more suitable.

Compound representation of causal fault-symptom relationships, or the so-called compound probability, also need to be defined to calculate the noisy-max CPT:

$$
c_{s}^{f_{i}^{n}}=P\left(S=s \mid f_{n}^{i}\right)
$$

This compound probability means the probability that fault $F_{n}$, when taking the value $f_{n}^{i}$, raises the symptom severity $S$ to $s$. In other words, a fault becoming more sever also raises the possibility of a more sever symptom. The usage of compound probability is the core of the noisy-max model. It simplifies the conditional probabilities, but at the same time brings in the limitation of ordinal symptom states.

Using leak probabilities and compound probabilities, the CPT can be calculated by using the following equations:

$$
P(S \leq s \mid f)=C_{s}^{L} \cdot \prod_{i} C_{s}^{f_{i}^{n}}
$$

and,

$$
P(s \mid F)= \begin{cases}P(S \leq s \mid f)-P(S \leq s-1 \mid f) & \text { for } s \neq s_{\text {min }} \\ P(S \leq s \mid f) & \text { for } s=s_{\text {min }}\end{cases}
$$

Where $C_{s}^{L}$ and $C_{s}^{f_{i}}$ are accumulative parameters of $c_{L}$ and $c_{s}^{f_{i}}$ by means of:

$$
C_{s}^{L}=\sum_{z_{L} \leq s} C_{z_{L}}^{L} \text { and } C_{s}^{f_{i}}=\sum_{z_{i} \leq s} c_{z_{i}}^{f_{i}^{n}}
$$


For $\mathrm{n}$ faults each with $i$ states, and each fault with $m$ releated symptoms with $k$ symptom states, the size of the conditional probabilities that need to be defined when using the noisy-max model instead becomes $n[i k(m-1)+k m]$. Since for most symptoms, the leak probabilities can share same default values, reducing of the number of definitions to $n i k(m-1)+m$. This means defining the CPT is no longer exponentially complicated, and for a binary fault $F_{1}$ with two related three-state symptoms $S_{1}, S_{2}$, the number of manual inputs is decreased from 18 to 9 . To further reduce this complexity, templates of compound probabilities and leak probabilities can be established, further reducing the total number of manual inputs to 3. Still, some heuristic inputs are needed, and future improvements will be addressed in Chapter 5.

\subsection{Fault Evaluation Agent}

As discussed in Chapter 2, the author favors the usage of building performance simulation (BPS) tools to evaluate fault impacts. To use BPS tools to predict fault impacts, a baseline model needs to be established for each building. Ideally, this model is either passed down from the design stage or created during a previous retrofit analysis. If the baseline model is not available, however, it is possible to create calibrated models manually or converted from building information models (BIM), or optimized from a meta-model as proposed by Eisenhower et al. [172]. This thesis assumes a baseline model is readily available and will not delve into the process of how to create one.

The challenge of using BPS to evaluate fault impacts is to determine how to quantitatively translate symptoms caused by a fault to specific inputs inside a BPS model. This involves three major steps: 
1. Identify symptoms directly caused by a diagnosed fault. These causal relationships are already defined in the DBN used for fault diagnostics. The fault evaluations agents use these casual relationships to determine which symptoms are needed for assessing diagnosed faults.

2. Quantify the severity of the symptom. Since these symptoms are usually deviations from their typical values, this can be achieved through comparing sensor measurements and estimated parameters to their expected values. The fault detection agents already provide such symptom quantification.

3. Map symptoms to specific inputs in BPS tools. This is the primary challenge of using specific BPS tools for fault evaluation. The authors select some of these mappings in EnergyPlus based on previous research [106], [173], [174] and knowledge.

\subsubsection{Symptom mapping}

The causal relationships between faults and symptoms are used previously to establish the DBN for diagnostics. In this section, a more concise form of algebraic representation denoting these relationships is used. The relationships developed in this article are based on typical faults and symptoms identified by previous research [39], [50], [90], [114], [147].

Table 3-4 shows examples of some typical faults and symptoms in air handling unit (AHU) and variable air volume (VAV) terminal faults using qualitative influence relationships described 
above. Symptoms quantified from the previous steps can be translated into BPS inputs. Most directly observed symptoms can be translated to specific BPS inputs using their raw values, such as AHU supply air pressure, thermostat setpoint, etc. On the other hand, for other symptoms that cannot be translated to BPS inputs, an indirect mapping approach needs to be adopted. Examples of direct and indirect mappings are shown in Table 3-5. This indirect mapping is required for most of the symptoms quantified by parameter estimations since the models used for the parameter estimation process are usually different from the first principle models used in the EnergyPlus model.

Table 3-4 Example of typical faults in building systems

\begin{tabular}{cccc}
\hline Equipment & Fault & Description & Relationship \\
& $F_{s f 1}$ & Belt slippage & $I+\left(S_{s f 1}, F_{s f 1}\right)$ \\
\multirow{3}{*}{ Supply Fan } & & & $I+\left(S_{s f 2}, F_{s f 1}\right)$ \\
& & & $I-\left(S_{s a 1}, F_{s f 1}\right)$ \\
\cline { 2 - 4 } & $F_{s f 2}$ & Decrease in motor efficiency & $I+\left(S_{s f 1}, F_{s f 1}\right)$ \\
\cline { 2 - 4 } & $F_{s f 3}$ & Overall failure & $I-\left(S_{s a 1}, F_{s f 1}\right)$ \\
\hline Heating coil & $F_{h c 1}$ & Fouling & $I-\left(S_{h r 1}, F_{h c 1}\right)$ \\
\hline Cooling coil & $F_{c c 1}$ & Fouling & $I-\left(S_{c r 1}, F_{c c 1}\right)$ \\
\hline \multirow{2}{*}{ Dampers } & $F_{r d 1}$ & Return air damper stuck closed & $I-\left(S_{r a 1}, F_{r d 1}\right)$ \\
\cline { 2 - 4 } & $F_{o d 2}$ & Outdoor air damper stuck open & $I+\left(S_{o a 1}, F_{o d 1}\right)$ \\
\hline \multirow{2}{*}{ VAV Terminal } & $F_{v h v 1}$ & Reheat valve stuck closed & $I-\left(S_{v s a 2}, F_{v h v 1}\right)$ \\
& & & $I-\left(S_{v r h 1}, F_{v h v 1}\right)$ \\
& & & $I-\left(S_{z t 1}, F_{v h v 1}\right)$ \\
\cline { 2 - 4 } Zone Level & $F_{v d 1}$ & Damper stuck open & $I+\left(S_{v s a 1}, F_{v d 1}\right)$ \\
\cline { 2 - 4 } & $F_{t s t 1}$ & Thermostat temperature positive offset & $I-\left(S_{z h 1}, F_{t s t 1}\right)$ \\
\hline \multirow{2}{*}{$F_{l t 1}$} & Lighting burn out & $I-\left(S_{z l 1}, F_{l t 1}\right)$ \\
& & & $I-\left(S_{z l 2}, F_{l t 1}\right)$ \\
\hline
\end{tabular}

This challenge of indirect mapping can be achieved by applying a common statistical model to the symptom and the corresponding BPS model input, then use a statistical value as an intermediary to translate the symptom severity to a specific BPS input. The authors then use the normal distribution to describe these values in terms of mean and standard deviation. In truth, 
Table 3-5 Examples of symptom mappings to EnergyPlus

\begin{tabular}{cccc}
\hline Symptom & Mapping in EnergyPlus & $\begin{array}{c}\text { Modelling } \\
\text { level }\end{array}$ & Direct Mapping \\
\hline $\boldsymbol{S}_{\boldsymbol{s f 1}}$ & Supply fan total efficiency & AHU & No \\
\hline $\boldsymbol{S}_{\boldsymbol{s a 1}}$ & Maximum supply air flow rate & AHU & Yes \\
\hline $\boldsymbol{S}_{\text {zi1 }}$ & Design infiltration rate & Zone & No \\
\hline
\end{tabular}

there might be better statistical distributions to describe different variables or parameters.

However, such investigation warrants a separate research.

\subsubsection{Multi-level simulation using model reduction}

Building systems operate at different scales. For example, while a VAV terminal only affects the zone it conditions, some faults can propagate through the hierarchy of building systems, i.e. a fault in the AHU can affect all the zones it conditions. Thus, it is useful to simulate faults at different levels of detail. For example, it is usually redundant to use a whole building model when a fault is only affecting a specific thermal zone. Thus, before mapping the symptoms into BPS inputs, the level of simulation needs to be defined to use an appropriate model. The authors define three levels of simulation for fault evaluation: zone level, AHU level, and building level. As its name suggests, zone level simulation only uses a zone model to evaluate faults contained within a thermal zone. AHU level analysis is responsible for faults inside an air handling unit affecting all the zones it conditions; and building level faults require the highest order of simulation which affects the performance of a whole building, such as hot water loop faults. The 
specific definition of which level is required to be simulated will be defined inside the symptomBPS input mapping section.

Since AFDD is a time-sensitive task, it is equally important to provide fault evaluations to the operators promptly. This requires short simulation time of BPS models.

For zone-level simulation this is readily achievable, however, for AHU-level or building-level models, some form of model order reduction is required to achieve faster simulation time. There are multiple approaches to BPS model order reduction; the most common one is using surrogate models. This usually requires the training of a black-box model to cover the parameter space of interest, in this case, the parameter space contains all the potential fault inputs. Examples of this BPS model order reduction approach include a surrogate model developed in OpenStudio [175], and the use of support vector regression as a surrogate model [172]. This approach is usually applied in building design optimization. However, for fault evaluation the parameter space could be much larger than a multi-criteria design optimization, this means to train a machine learning model as surrogate requires an enormous amount of simulation to be performed beforehand. Another approach is to selectively eliminate objects in the BPS model so that it approximates the original model, usually called selective node elimination in model order reduction research. Compared to surrogate models, this approach requires much less training data, making it more realistic to achieve. Examples of applying selective node elimination in BPS include using graphic theory to simplify building geometry [176], and applying Koopman operator to merge similar thermal zones [177]. The model order reduction method used in this 
research for AHU-level and building-level fault evaluation is called model-reduce-cluster, which is discussed in detail in the next section.

\subsubsection{Model-Cluster-Reduce Method for Model Reduction}

The Model-Cluster-Reduce model reduction pipeline is composed of three main steps (as summarized in Figure 3-13). The procedure for each step is explained as follows:

1. The model step captures the characteristics of the original thermal zones by parameterizing a physics-based or statistically-driven model. This step produces a reduced number of parameters from the analytical model used for clustering. Besides physical parameters, normalized heating/cooling profile could also be used to characterize the zones as an abstracted parameter. The thermal parameters produced in this step should avoid using specific inputs to a BPS tool to make this approach more general.

2. The cluster step uses the parameters obtained from the previous step to group thermal zones automatically using clustering techniques. Then the resulting centers of each cluster group become archetype zones needed for model reduction.

3. The archetype zones are used to produce the reduced model with a scale factor. The scale factors are calculated from attributes such as floor area and volume. If the boundary condition of the archetype zones' surfaces is not exterior or another archetype zone, it will be converted to adiabatic. 


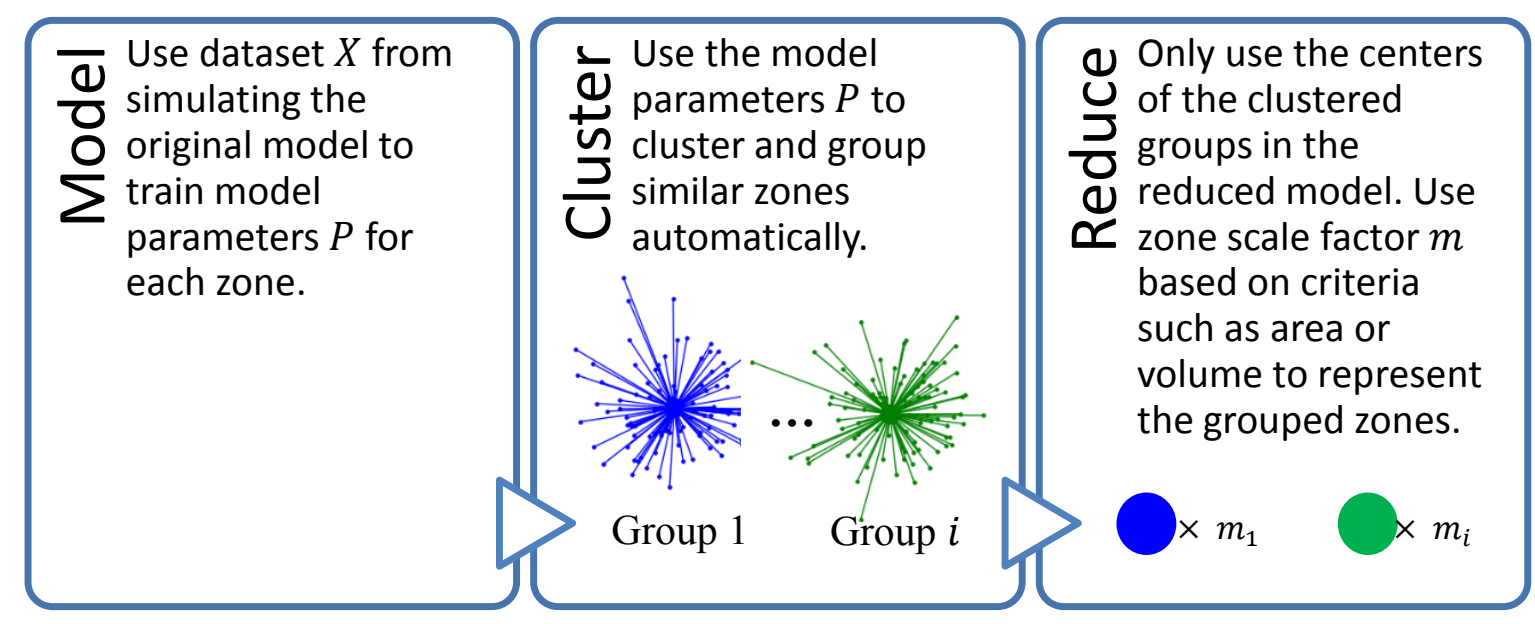

Figure 3-13 Schematics of the Model-Cluster-Reduce Pipeline [178]

The time series of hourly heating/cooling load of each thermal zone is used in the model step as an abstraction of the thermal characteristics. The heating/cooling load is a superposition of all the heat transfer components occurring inside each thermal zone, which in theory, may be able to provide enough information to represent the uniqueness or affinities of this zone. In this model approach is used; the model parameters can be calculated by simply normalizing the heating/cooling load by zone floor area:

$$
P=\frac{\text { zone hourly heating/cooling load }}{\text { zone floor area }}
$$

During the clustering step, it is imperative for the clustering algorithm to use real thermal zones from the original model instead of arithmetic means as the centre of each cluster. This is due to the limitation of the next step of the pipeline -- the centre of each cluster will be used as an archetype zone to represent the whole group of zones. Since arithmetic centres are only based on reduced parameters, they cannot be converted back to BPS inputs. Only a handful of clustering algorithms are based on medians or centroids; examples include k-centre and Affinity 
Propagation. Other common clustering techniques such as k-means and DBScan should be modified such that objects closest to the arithmetic centres become cluster centres. Affinity Propagation (AP) [179] is chosen since its characteristics fit the requirements in this research. AP performs clustering by exchanging messages between data points recursively until a formation of high-quality clusters emerges. Compared to other clustering algorithms such as kcentres, AP does not require a predefined number of clusters to be classified and is better at handling a large number of clusters, and less prone to random initialization issues [179]. The original paper [179] can be referenced for more details about AP.

Since in the previous model step normalized heating/cooling load is directly used to represent the thermal zone, this clustering task becomes a time series clustering problem. Euclidean distance is still used in the similarity calculations, which is calculated to determine the cluster formation. Other time series distances that can be used as similarities such as dynamic time warping and cross-correlation are not suitable. For detailed information about time series clustering and the selection of affinity function, the original paper by Frey and Dueck [179] could be referenced. After the modelling and clustering step, each archetype zone is then used to represent other zones inside its cluster by using scale factors. The scale factor can be calculated from properties such as floor area, volume, and occupied floor area. The scale factor is the ratio of the value of the chosen property for the original zone to the value of the property for the archetype zone. In most buildings where most thermal zones share the same height, floor area would be the best candidate to calculate the multipliers since many parameters in the model are directly connected 
to the floor area. For instance, the scale factor would be two if the total area of zones of the cluster is double the area of their archetype.

If floor area is selected for calculation, scale factor $\mu$ for archetype zone $\alpha$ and its child zones $\beta_{\alpha}$ can be calculated by:

$$
\mu_{\alpha}=\frac{\sum A_{\beta_{\alpha}}}{A_{\alpha}}
$$

During the model reduction process, only objects related to the archetype zones are kept. All interior surfaces of the archetype zones which have a boundary condition of another thermal zone are changed to adiabatic. Shading surfaces are kept in the reduced model. Besides nonarchetype thermal zones that need to be removed, all other objects such as schedules, equipment loads and HVAC components not belonging to the archetypes are removed from the model to reduce the model loading time. Self-shading is ignored, however, if self-shading becomes critical, deleted exterior surfaces can be converted to shading surfaces.

The above-mentioned Model-Cluster-Reduce model reduction method is developed for general building energy model optimization and parametric simulation use. It is also available as an open source project on GitHub, as in Appendix D . For more detail about this model reduction method and a more generalized architecture, the original article published by the author on Journal of Building Performance Simulation can be referenced [178]. A preliminary study was also published at the $3^{\text {rd }}$ Asia International Building Performance Simulation Association Conference (ASIM 2016) [180].

\subsection{Summary}

This chapter provided theoretical formulations of the proposed fault detection, diagnostics and evaluation framework. It started from the overall framework design to the methodologies behind 
each functional agent. The rationale behind the selection of some of the specific techniques such as probabilistic evidence, Dynamic Bayesian Networks, as well as the leaky noisy-max model, were explained. Some components of the AFDDE framework, such as constrained dual EKF and model-cluster-reduce model reduction method, were generalized and published as separate articles. The next results chapter will introduce the case studies used to demonstrate the proposed framework, as well as specifics of how they are implemented in zone-level and AHU-level studies. 


\section{Chapter: Results}

A total of four case studies composed of 24 individual fault cases were conducted to demonstrate the proposed AFDDE framework. One simulation-based and one measurement-based dataset were created for zone-level and AHU-level case studies. Due to limited sensing capabilities, fault evaluation could not be validated for the measurement-based case studies, since energy usage, cost, and thermal comfort were not directly monitored. However, fault evaluations were still tested and compared for the simulation-based case studies.

Before delving into the results, the definition of several vital metrics used for evaluating the effectiveness of an AFDDE system will be explained. These metrics include false positive rate, false negative rate and fault diagnose time. Figure 4-1 provides an example of how these metrics, and how they affect the reliability of an AFDD system.

A false positive is defined by two consecutive fault probabilities higher than the 0.6 thresholds during fault-free operation. A false negative is defined by fault probability becoming lower than 0.6 after initial data accumulation stage during faulty operation. The 0.6 threshold for fault probability in this research is defined arbitrarily and is used to represent a "faulty" state in general. The diagnose time, measured by time step, is the amount of time required for the AFDDE system to correctly diagnose the fault after occurring, and in theory, a diagnose time of 1 means the fault is instantly diagnosed after the data is processed. In many cases, evidence needs to be accumulated until a proper fault is diagnosed due to the nature of DBN used in this framework.

Table 4-1 provides an overview of the case studies' results. For each case study, aggregated false positive rates and false negative rates were reported. In general, the false positive rates and false negative rates were quite low, within a 5\% probability. The false positive rates are lower than 
false negative rates, which is preferable and will decrease the overall number of false alarms at the cost of a less sensitive AFDDE system. Still, a $0 \%$ false positive rate and false negative rate should be the target for all AFDDE frameworks.

The occurrence of false positives is further mitigated by the fact that the proposed AFDDE system treats the diagnosed faults continuously, instead of raising alarms every time the fault probability exceeds a certain threshold (in this research 0.6 ). So, in most cases, these false alarms

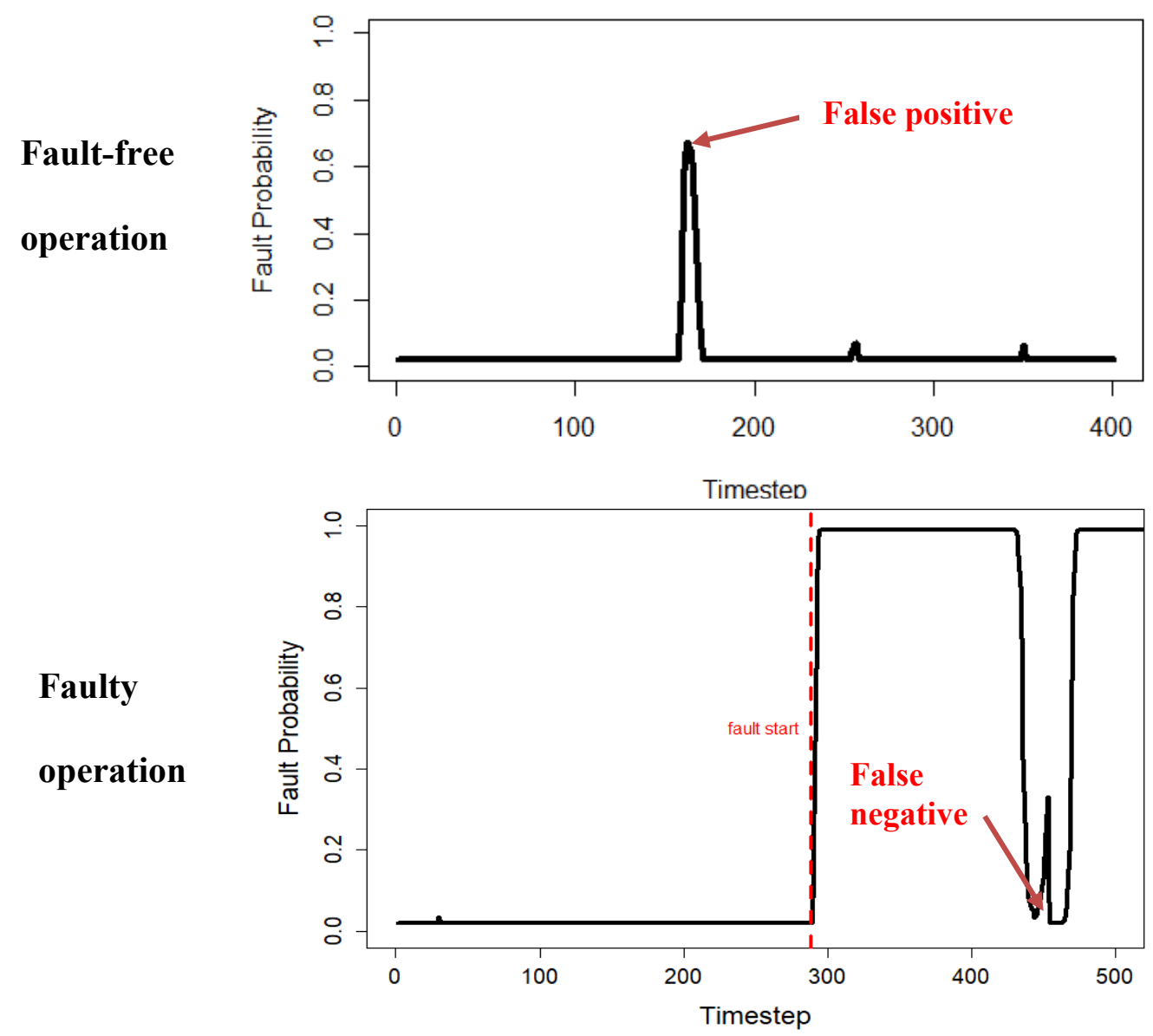

Figure 4-1 Example of the metrics used to evaluate the FDDE framework 
will be removed once the incorrectly predicted high fault probability moves back to fault-free values based on further measurements.

Note the difference in time step used for these case studies does not significantly affect the outcomes, so in future applications, it is preferable to have a smaller time step setup to make the AFDDE system more responsive. However, in real applications, the selection of time step should be carefully evaluated based on the building system, network limitations and computational resources. Detailed outcomes of each case study will be carefully examined in the following sections.

Table 4-1 Overview of the case study results

\begin{tabular}{|c|c|c|c|c|}
\hline Case Study Name & $\begin{array}{c}\text { Number of } \\
\text { fault cases }\end{array}$ & Time step & $\begin{array}{c}\text { False positive } \\
\text { rate }\end{array}$ & $\begin{array}{c}\text { False Negative } \\
\text { Rate }\end{array}$ \\
\hline $\begin{array}{c}\text { Shoebox } \\
\text { simulation }\end{array}$ & 5 & 15 minutes & $2.6 \%$ & $4.8 \%$ \\
\hline$\underline{\text { Measured office }}$ & 4 & 10 minutes & $1.8 \%$ & $3.5 \%$ \\
\hline$\underline{\text { ASHRAE RP-1312 }}$ & 10 & 1 minute & $0.4 \%$ & $1.6 \%$ \\
\hline$\underline{\text { AHU simulation }}$ & 5 & 15 minutes & $0.6 \%$ & $1.2 \%$ \\
\hline
\end{tabular}




\subsection{Zone-level Case Studies}

Two zone-level case studies are presented in this section. The implementation of the zone-level AFDDE system will be discussed first, followed by the outcomes from the case studies.

Thermal zones or rooms in buildings are affected by a large number of interconnected processes, such as heat transfer and mass with the exterior environment as well as the HVAC system, internal gains from the interior equipment, and so on. They often lack sufficient sensing equipment to appropriately monitor every single process. This is where the proposed parameter estimation scheme introduced earlier becomes useful, as the sequential dual estimation algorithm can be implemented inside a controller without a heavy computation toll. The reduced model used for this parameter estimation task is shown in Figure 4-2. It is composed of a total number of five parameters, and the model function is:

$$
f=\left[\begin{array}{c}
T_{i}+\left(T_{o u t}-T_{i}\right) \phi_{1}+S_{o c c} \phi_{2}+E_{\text {light }} \phi_{3}+ \\
Q_{v a v}\left(T_{v a v}-T_{i}\right) \phi_{4}+\phi_{5}
\end{array}\right]
$$

where $T_{\text {out }}$ is the outdoor temperature, $S_{\text {occ }}$ is the binary occupancy state, $E_{\text {lux }}$ is the illuminance level measured on the ceiling, $Q_{v a v}$ is the VAV box supply air flow rate and $T_{v a v}$ is the supply air temperature.

This makes each of the parameters related to a particular physical characteristic of the zone as follows:

1. $\phi_{1}$ : related to indoor - outdoor heat transfer

2. $\phi_{2}:$ related to internal heat gain cuased by the occupant

3. $\phi_{3}$ : related to solar and lighting heat gain

4. $\phi_{4}$ : related to heat gain/loss from the HVAC system

5. $\phi_{3}:$ related to constant internal load (phantom load) 


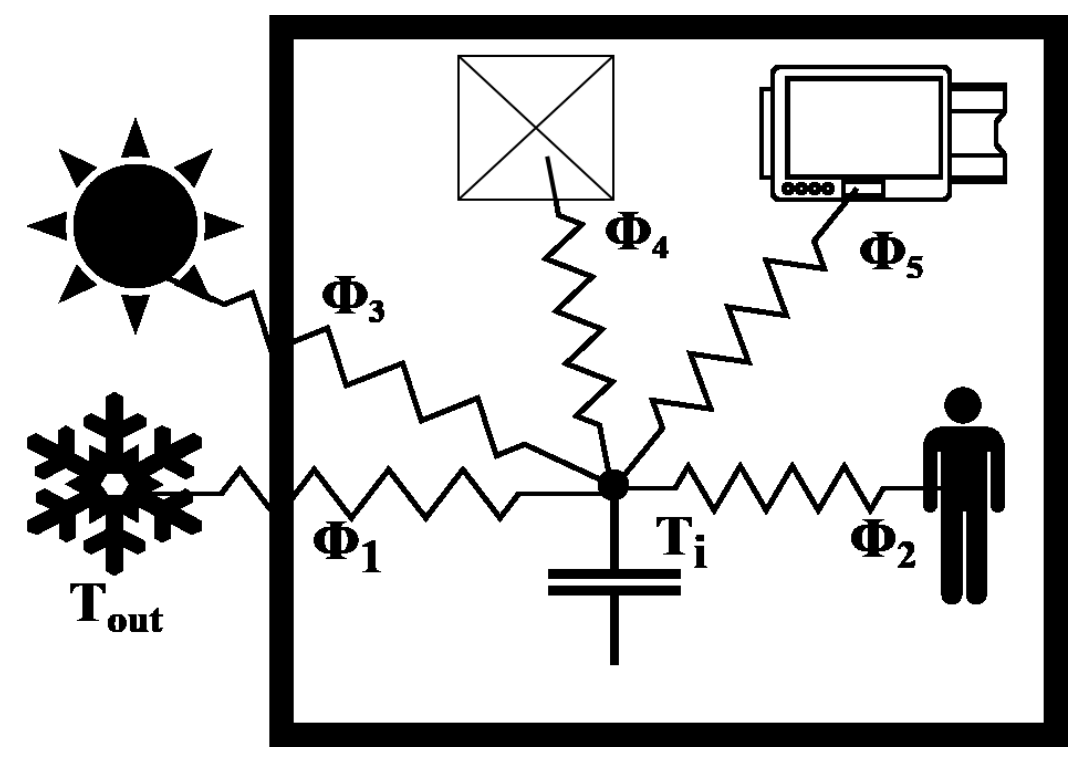

Figure 4-2 Reduced model used for zone parameter estimation

The model was initiated with a two-week training data, and then used to sequentially update its parameters. The normal range of the parameters used for symptom generation and fault detection is updated by a trailing exponential weighted average with a moving window of two weeks. Table 4-2 further shows a list of symptoms detected and calculated inside the zone level fault detection agent. A total number of 10 symptoms are included in this implementation. Only four of these are directly observed sensor inputs, while the results are calculated from parameter estimation mentioned above or from empirical functions. To come with the reference ranges for the estimated parameters, one month of moving window is used to calculate the means and standard deviations during normal operations. The ranges of the parameter thresholds are updated recursively every two weeks. 
Table 4-2 List of symptoms generated inside the zone fault detection agent

\begin{tabular}{|c|c|c|c|}
\hline Symptom & Description & $\begin{array}{c}\text { Direct } \\
\text { Observation }\end{array}$ & $\begin{array}{c}\text { Logistic Regression } \\
\text { Template }\end{array}$ \\
\hline $\mathbf{S}_{t s t 1}$ & Thermostat setpoint difference & Yes & High \\
\hline $\mathbf{S}_{t s t 2}$ & Thermostat setpoint & Yes & High \\
\hline$S_{\text {vav1 }}$ & Supply air temperature & Yes & High \\
\hline$S_{\text {vav2 }}$ & Supply air pressure & Yes & High \\
\hline$S_{z, p 1}$ & Estimated infiltration rate & No & Low \\
\hline $\mathbf{S}_{z, p 2}$ & $\begin{array}{l}\text { Estimated phantom load } \\
\text { parameter }\end{array}$ & No & Low \\
\hline $\mathbf{S}_{z, s 2}$ & Cooling Sensitivity & No & Low \\
\hline$S_{z, p 3}$ & $\begin{array}{l}\text { Estimated occupied load } \\
\text { parameter }\end{array}$ & No & Low \\
\hline$S_{z, p 4}$ & $\begin{array}{l}\text { Estimated VAV system } \\
\text { efficiency }\end{array}$ & No & Medium \\
\hline$S_{Z, s 1}$ & Heating sensitivity & No & Low \\
\hline
\end{tabular}

The fault-symptom relationships defined in the zone level AFDDE system is shown in Table 4-3. A total number of six faults are implemented for the zone-level cases studies in this thesis. Note for $F_{t s t 1}, F_{t s t 2}$ and $F_{z 2}$, only one symptom is linked to the fault, making the overall structure of the DBN less complex.

Table 4-3 Faults implemented at the zone level FDDE system

\begin{tabular}{ccc}
\hline Fault & Description & Relationship \\
\hline $\boldsymbol{F}_{\boldsymbol{t s t} \mathbf{1}}$ & Incorrect thermostat setpoint & $I\left(S_{t s t 2}, F_{t s t 1}\right)$ \\
\hline $\boldsymbol{F}_{\boldsymbol{t s t 2}}$ & Thermostat sensor bias & $I\left(S_{z, p 4}, F_{t s t 2}\right)$ \\
\hline $\boldsymbol{F}_{\text {vav1 }}$ & VAV reheat valve stuck & $I\left(S_{\text {vav } 1}, F_{\text {vav } 1}\right)$ \\
& & $I\left(S_{t s t 1}, F_{\text {vav1 } 1}\right)$ \\
\hline $\boldsymbol{F}_{\text {vav2 }}$ & VAV damper stuck & $I\left(S_{\text {vav } 2}, F_{\text {vav } 2}\right)$ \\
\hline $\boldsymbol{F}_{\boldsymbol{z} \mathbf{1}}$ & Unintended window opening & $I+\left(S_{z, s 1}, F_{z 1}\right)$ \\
& & $I+\left(S_{z, s 2}, F_{z 1}\right)$ \\
& & $I+\left(S_{z, p 1}, F_{s 1}\right)$ \\
\hline $\boldsymbol{F}_{\mathbf{z 2}}$ & Unexpected high phantom load & $I+\left(S_{z, p 2}, F_{z 2}\right)$ \\
\hline
\end{tabular}




\subsubsection{Shoebox Model Simulation}

The first case study uses a shoebox model to simulate fault cases on a single zone level. The simulation was conducted in EnergyPlus version 8.5 [181] using a south-facing shoebox model with a $40 \%$ window to wall ratio. The model is located in Ottawa, Ontario, Canada. The dimension of the shoebox model is $5 m \times 5 m \times 3 m$. All interior surfaces are assumed to be adiabatic. The zone is conditioned by a packaged VAV system. The lighting sensor is in the center of the zone on the work surface. Artificial sensor noise is also added to the simulated data to mimic sensor noise as Gaussian (white) noise [20][182]. The addition of the sensor noise could challenge the proposed method's ability to handle uncertain data to produce reliable fault diagnosis. Note since the occupancy sensor provides a binary reading, no Gaussian noise is added to the occupancy state.

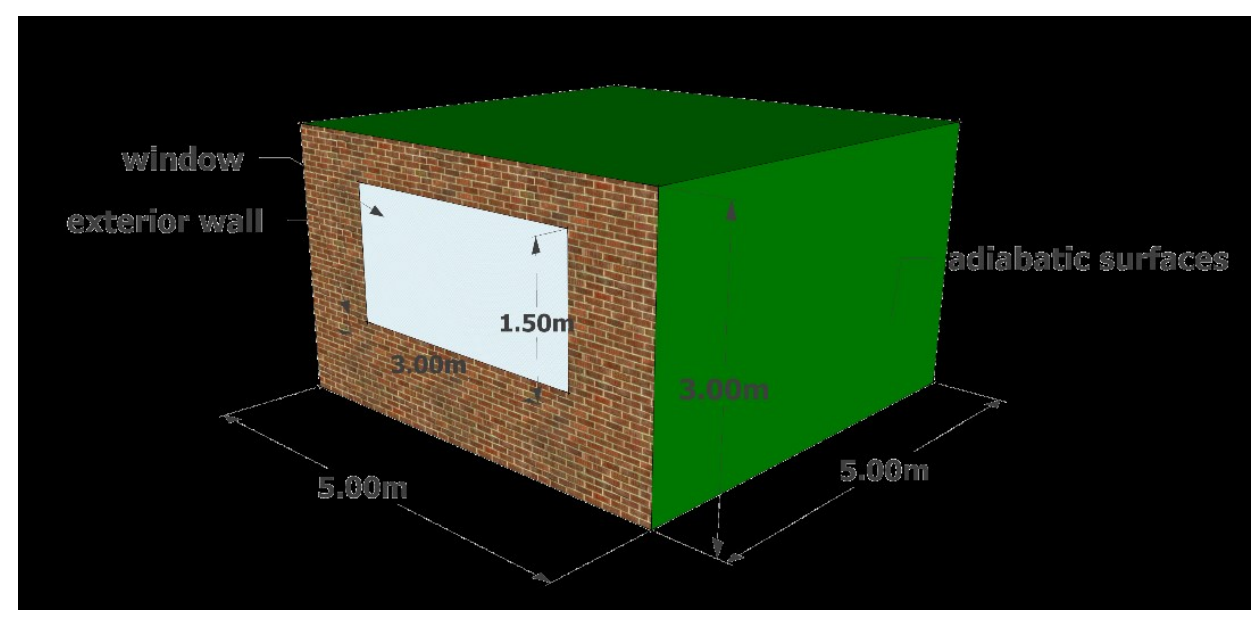

Figure 4-3 Dimension of the shoebox model

A total number of six faults are simulated during the heating season. The fault cases are created by changing their corresponding model inputs. One month of normal operation is simulated first 
to provide training dataset, followed by two weeks of faulty operation data. The faults are described as follows:

1. Unintended window opening. This fault can cause increased air infiltration inside a thermal zone, and lead to increased energy use to condition its temperature. In worst case scenarios this may also cause equipment damage due to extreme temperature and humidity since most HVAC systems are not designed to handle long-term window openings during the heating and cooling seasons. This fault is simulated by doubling the design infiltration rate inside the EnergyPlus model, which serves as a conservative estimation since in most cases infiltration area would be increased significantly.

2. Stuck closed VAV supply air damper. It is possible to have VAV damper motor to malfunction, causing the supply air damper to be stuck at the closed location. This may lead to insufficient heating, cooling and fresh air supply. This fault is modelled by changing the maximum supply air flow of the VAV terminal to $10 \%$ of its design value after the fault occurred.

3. Stuck closed VAV reheat valve. Similar to the previous fault, this causes low supply air temperature, leaving the zone potentially under-heated. This fault is monitored by the supply air temperature as well as the difference to the actual set point for this temperature. When the reheat valve is closed the reheat availability is set to zero in EnergyPlus.

4. Biased thermostat temperature reading. A biased thermostat can cause underheating/overheating issues. In this case study, a positive bias is added, meaning the measured temperature is higher than the actual temperature, which could lead to underheating issues. A static bias of $2^{\circ} \mathrm{C}$ is added to the thermostat fault object of 
EnergyPlus. This fault is hard to diagnose and may require feedback from the occupant to identify it.

5. Unwanted phantom electricity load. While this fault does not likely have direct comfort related consequences unlike the previous faults, it increases unintended electricity usage and causes inefficiency. The symptom related to this fault is the estimated phantom load parameter obtained from the inverse modelling step in the fault detection agent.

Table 4-4 summarizes the fault diagnostics results. Figure 4-4 shows a snapshot of fault probabilities for all fault cases; the faults start at time step 100. The diagnose time is the number of time steps required for the FDDE system to report a fault probability of higher than 0.6 thresholds. Terminal fault belief is the final fault probability after the calculation stabilizes. Individual false negative rates are also reported. For all fault cases, the terminal fault belief reached maximum value $-99.9 \%$, indicating the end-users would receive reports with a strong belief that these faults occurred. The two faults relying on estimated parameters - unintended window opening fault and unwanted phantom load fault, have the highest false negative rate and require a longer time to be detected. This is probably caused by the modelling errors occurred during the parameter estimation process. If more sensitive and reliable parameter estimation procedures are to be established in the future, the detection time and false negative rates will likely be decreased. 
Table 4-4 Fault diagnostics results for simulation-based zone-level case study

\begin{tabular}{|c|c|c|c|c|}
\hline & Fault Name & $\begin{array}{l}\text { Diagnosis Time } \\
\text { (Time Steps } \\
\text { after fault } \\
\text { occurs) }\end{array}$ & $\begin{array}{c}\text { Terminal Fault } \\
\text { Belief }\end{array}$ & $\begin{array}{c}\text { False Negative } \\
\text { Rate }\end{array}$ \\
\hline$\underline{1}$ & $\frac{\text { Unintended Window }}{\text { opening }}$ & 6 & $99.9 \%$ & $4.2 \%$ \\
\hline$\underline{2}$ & $\frac{\text { VAV reheat valve stuck }}{\underline{\text { closed }}}$ & 1 & $99.9 \%$ & $0 \%$ \\
\hline$\underline{3}$ & $\frac{\text { VAV damper stuck }}{\underline{\text { closed }}}$ & 5 & $99.9 \%$ & $1.6 \%$ \\
\hline$\underline{4}$ & Static thermostat bias & 2 & $99.9 \%$ & $0 \%$ \\
\hline$\underline{5}$ & Unwanted phantom load & 63 & $99.9 \%$ & $4.8 \%$ \\
\hline
\end{tabular}

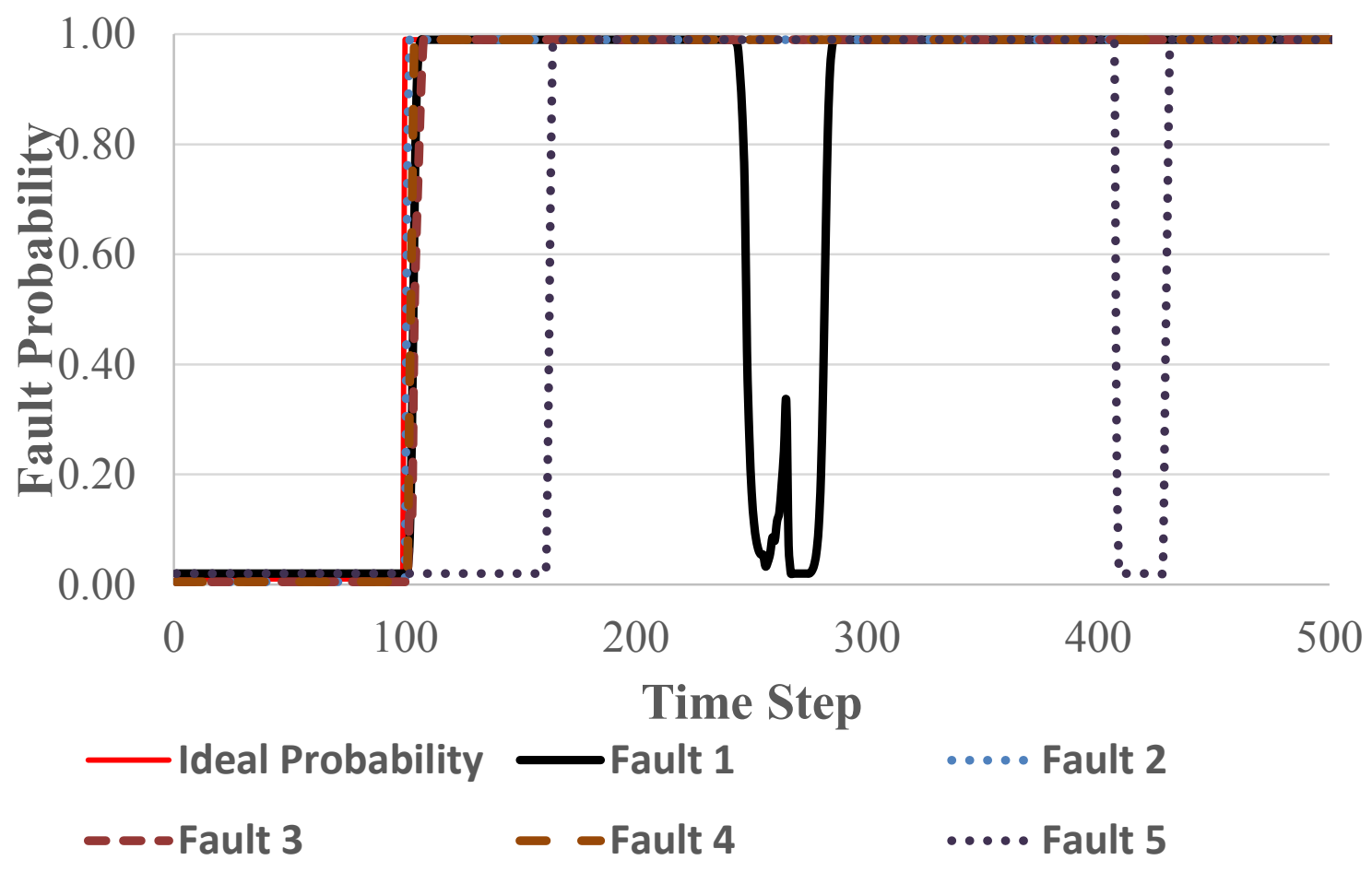

Figure 4-4 Example of the FDD results for simulated zone study; the faults start at 100 time step 
Table 4-5 demonstrates fault evaluation results for this zone-level simulation generated case study. Unfortunately, not all faults can be evaluated since not all symptoms can be quantified properly. In this case, the thermostat bias fault does not have a quantifiable symptom from the available measurements; and the increased phantom load could just be part of the normal operation. In general, the fault evaluation results fall within the same order of magnitude with the actual impacts, and the uncertainty analysis for the window opening fault also showed the median of estimated impact close to the actual impact. However, estimated thermal comfort impact for stuck closed VAV reheat valve resulted in an over $50 \%$ over-prediction. This can be explained by the fact that the thermal comfort model could cause an extra layer of modelling error on top of the existing energy simulation, resulting in less reliable estimates. Still, the author would like to argue that by quantitative assessments of faults is still superior to the operators for supporting maintenance decision-making. Fault evaluation will be discussed further in Chapter 5.

Table 4-5 Fault Evaluation results for simulation-based zone-level case study

\begin{tabular}{|c|c|c|c|c|c|c|}
\hline \multirow{2}{*}{ Fault Name } & \multicolumn{2}{|c|}{ Energy (kWh) } & \multicolumn{2}{c|}{ Comfort (PPD) } & \multicolumn{2}{|c|}{ Cost } \\
\cline { 2 - 7 } & Actual* & Evaluated & Actual $^{*}$ & Evaluated & Actual $^{*}$ & Evaluated \\
\hline Window open & 4,780 & $2,276-7,966$ & $/$ & $/$ & $\$ 478$ & $\$ 228-\$ 797$ \\
\hline VAV reheat valve stuck closed & $/$ & $/$ & $53 \%$ & $85 \%$ & $/$ & $/$ \\
\hline VAV damper stuck open & 9,305 & 9,861 & $/$ & $/$ & $\$ 930$ & $\$ 986$ \\
\hline
\end{tabular}

* simulated with EnergyPlus for this case study. 


\subsubsection{Single Zone: Experiment}

The second case study uses real measurements from offices inside an actual building. The Canal Building is a mixed-use academic building located at Carleton University campus in Ottawa, Canada. These offices are conditioned by a VAV system with reheat coils as well as radiant heat panels for perimeter heating. Four faults are studied in this case study, three of which occurred during the data collection process and had caused major damage to the built environment (see Figure 4-5). One fault, the static thermostat bias, is introduced artificially to the measured data afterwards. The data is collected at 10 minutes interval by the building automation system. A two-month dataset is used to train the normal operating range. Measurements available within this dataset include indoor air temperature, thermostat set point temperature, outdoor air temperature, VAV supply air temperature, VAV supply air pressure, occupancy (motion sensor) and control signals. Most of the symptoms in the zone level AFDDE implementation are monitored or could be estimated using the same reduced model from the previous case study. Unlike previous case study, sensor noise already exists, so no additional sensor noise was added. Moreover, the duration of faulty operation is shorter since all faults were remedied within a week. 


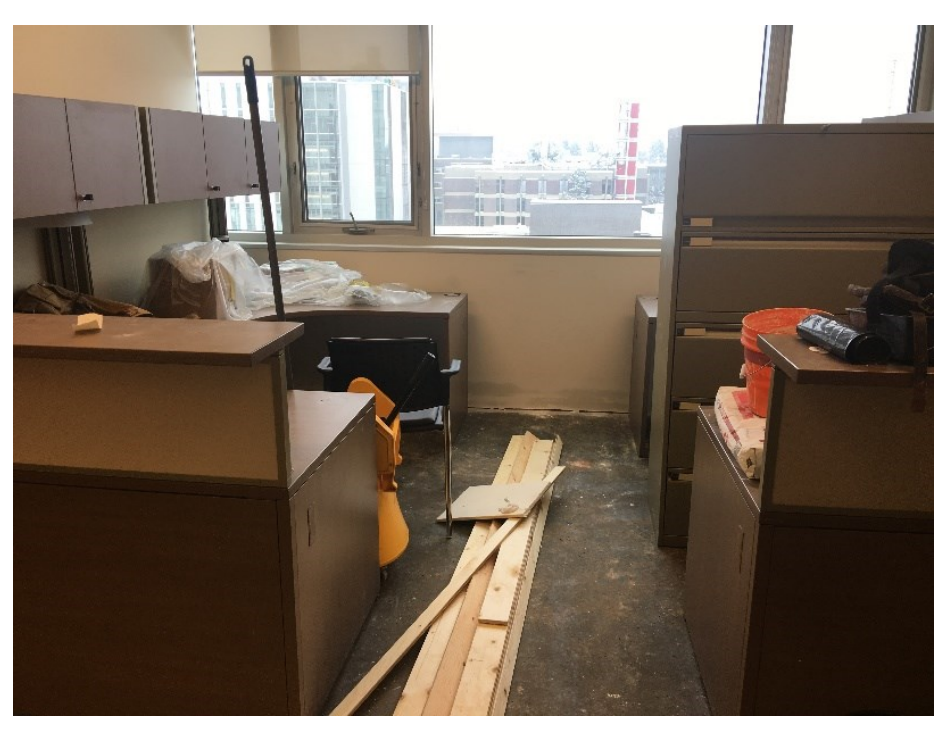

Figure 4-5 Image of an unintended window opening fault causing freezing pipes and water damage

Table 4-6 summarizes the FDD results from this case study. It is worth noting that since these faults were remedied in a short time, the duration of faults is much lower than in other case studies. The faults in this case study last from a couple of days (Fault 2) to five days (Fault 1). When compared to the previous simulation-based case study, the fault negative rates and false positive rates are much lower, while the detection time based on estimated parameters (Fault 1 and 4) are higher. The lower false negative and false positive rates can be explained that the sensor data may be more reliable than the simulated data with artificial noises. Still, the estimated parameters are slower to respond to the actual changes inside the zones, making the FDD less responsive. Especially for Fault 1 unintended window opening, the FDD system took 62 time steps, or about 6 hours, to properly diagnose the fault. Better techniques and models used for parameter estimation can help remedy this issue. Fault evaluation is not performed for this 
case study since there was no measured energy and cost impact for these individual zones to compare against.

Table 4-6 Summary of the FDD results from the measured zone-level case study

\begin{tabular}{|c|c|c|c|c|}
\hline & Fault Name & $\begin{array}{c}\text { Diagnosis } \\
\text { Time } \\
\text { (Time Steps) }\end{array}$ & $\begin{array}{c}\text { Terminal Fault } \\
\text { Belief }\end{array}$ & $\begin{array}{c}\text { False Negative } \\
\text { Rate }\end{array}$ \\
\hline$\underline{1}$ & $\underline{\text { Window open }}$ & 62 & $99.9 \%$ & $0 \%$ \\
\hline$\underline{2}$ & $\underline{\text { VAV reheat valve stuck closed }}$ & 1 & $99.9 \%$ & $0 \%$ \\
\hline$\underline{3}$ & $\underline{\text { VAV damper stuck closed }}$ & 4 & $99.9 \%$ & $0 \%$ \\
\hline$\underline{4}$ & $\underline{\text { Static thermostat bias }}$ & 26 & $99.9 \%$ & $0.7 \%$ \\
\hline
\end{tabular}

\subsection{AHU-level Case Studies}

Two AHU-level case studies are presented in this section, implementation of the AHU-level AFDDE system will be discussed first, followed by the outcomes from the case studies.

For the AHU level implementation, parameter estimation is also needed, but not as sophisticated as the zone-level counterpart since AHUs are usually well-equipped with sensors. The only parameters used in this application are equipment steady-state efficiency, calculated by the ratio between output quantity and input power. For example, to calculate the return fan efficiency, the ratio between return air flow rate and electrical power is used. As shown in Table 4-7, a total number of 12 symptoms are monitored, three of which are equipment efficiencies. Similar to zone-level implementation, two case studies are demonstrated for the AHU-level FDDE system. 
Table 4-7 Symptoms created for AHU-level implementation

\begin{tabular}{|c|c|c|c|}
\hline Symptom & Description & Direct Observation & $\begin{array}{c}\text { Logistic Regression } \\
\text { Template }\end{array}$ \\
\hline $\mathbf{S}_{c c 1}$ & $\begin{array}{c}\text { Chilled water flow } \\
\text { low }\end{array}$ & Yes & low \\
\hline $\mathbf{S}_{c c 2}$ & $\begin{array}{l}\text { Chilled water flow } \\
\text { high }\end{array}$ & Yes & low \\
\hline$S_{e a f 1}$ & Exhaust air flow low & Yes & Low \\
\hline$S_{o a f 1}$ & Outdoor air flow low & Yes & Low \\
\hline$S_{\text {raf1 }}$ & $\begin{array}{c}\text { Return air low flow } \\
\text { rate }\end{array}$ & Yes & Medium \\
\hline$S_{r f 1}$ & Return fan low speed & Yes & low \\
\hline$S_{s a t 1}$ & $\begin{array}{c}\text { Supply air temperature } \\
\text { low }\end{array}$ & Yes & Low \\
\hline$S_{b l 1}$ & $\begin{array}{l}\text { Boiler low output } \\
\text { temperature }\end{array}$ & Yes & Low \\
\hline$S_{b l 2}$ & Boiler low efficiency & No & Medium \\
\hline$S_{c l 1}$ & $\begin{array}{l}\text { Chiller low electric } \\
\text { efficiency }\end{array}$ & No & High \\
\hline$S_{r f 2}$ & $\begin{array}{l}\text { Return fan low } \\
\text { efficiency }\end{array}$ & No & High \\
\hline$S_{s a f 1}$ & Supply airflow low & Yes & Low \\
\hline
\end{tabular}

The causal relationships between the faults and symptoms are shown in Table 4-8. Seven faults in total are added to the AHU-level FDDE system. Some of these faults have three different states, for example, the chilled water valve stuck fault has the states of "stuck closed", "stuck open" and "fault-free".

Table 4-8 AHU-level fault-symptom relationships

\begin{tabular}{ccc}
\hline Fault & Description & Relationship \\
\hline $\boldsymbol{F}_{\boldsymbol{r f 1}}$ & Return fan low efficiency & $I\left(S_{t s t 2}, F_{t s t 1}\right)$ \\
\hline $\boldsymbol{F}_{\boldsymbol{c c 1}}$ & Chilled water valve stuck & $I\left(S_{z, p 4}, F_{t s t 2}\right)$ \\
\hline $\boldsymbol{F}_{\boldsymbol{o a 1}}$ & Outdoor air damper stuck & $I\left(S_{\text {vav1 }}, F_{\text {vav1 } 1}\right)$ \\
& & $I\left(S_{t s t 1}, F_{v a v 1}\right)$ \\
\hline $\boldsymbol{F}_{\boldsymbol{e a 1}}$ & Exhaust air damper stuck & $I\left(S_{\text {vav2 }}, F_{\text {vav } 2}\right)$ \\
\hline $\boldsymbol{F}_{\boldsymbol{a h u 1}}$ & Supply air leakage & $I+\left(S_{z, s 1}, F_{z 1}\right)$ \\
& & $I+\left(S_{z, s 2}, F_{z 1}\right)$ \\
& & $I+\left(S_{z, p 1}, F_{s 1}\right)$ \\
\hline $\boldsymbol{F}_{\boldsymbol{b l 1}}$ & Boiler low air intake & $I+\left(S_{z, p 2}, F_{z 2}\right)$ \\
\hline $\boldsymbol{F}_{\boldsymbol{c l 1}}$ & Chiller low efficiency & $I+\left(S_{z, p 2}, F_{z 2}\right)$ \\
\hline
\end{tabular}

\subsubsection{ASHRAE RP-1312}


For the AHU-level measurement-based case study, published experimental data from ASHRAE Research Project 1312 [183] is used. RP-1312 used two identical air handling units to generate faulty data set for the cooling season, heating season and spring season. One air handling unit is used to generate faulty operation data, and the other air handling unit is used to generate faultfree data as a control. Numerous sensors were installed and validated, making this dataset an ideal case study. Unfortunately, only the cooling season data and spring season data is used in this case study due to some measurement issues reported during the heating season. As mentioned in the methodology chapter, Zhao et al. [99] have previously used the same dataset to demonstrate the FDD results with an event-based Bayesian Network, which will be used as a direct comparison to the proposed method with the Dynamic Bayesian Network. Five types of AHU faults with various severities were used in this case study, making the total number of nine individual fault cases. Below is a brief description of each of the fault types:

1. OA damper stuck close: a stuck closed outdoor air damper with two possible severities fully closed and $40 \%$ open. This could lead to the lowered fresh air intake and eventually decrease the indoor air quality and potentially reduced free cooling.

2. EA damper stuck close: a stuck closed exhaust air damper also with two possible severities - fully closed and $40 \%$ open. This could lead to the decreased fresh air intake, increased supply air flow and deteriorated indoor air quality.

3. Return fan performance: a fully closed return fan and a lower performance return fan could cause the AHU not able to condition the whole building due to decreased air circulation.

4. Closed cooling coil valve: a fully closed or stuck at $15 \%$ opening cooling coil valve can lead to higher supply air temperature and overheating issues in the building. 
5. AHU leakage: air leakage along the supply air path could waste energy and not providing enough supply air flow rate to the building.

Results using the proposed FDDE system based on the ASHRAE RP-1312 database is shown in Table 4-9. Overall the fault diagnostics metrics are better compared to the previous case studies in this thesis, partially due to the simplicity of fault-symptom relationships, as most of these faults' symptoms are directly measured by the automation system. When compared to the results from another research using event-based Bayesian Network, the proposed DBN can provide more reliable diagnostics. Still, the AHU leakage fault cannot be adequately diagnosed due to limited data from this dataset since the air pressure after the economizer is not directly reported. The false negative rates and detection steps are also quite low, with only a $1.6 \%$ overall false negative rate. Unfortunately, these metrics were not reported by Zhao et al., so a direct comparison of the $\mathrm{BN}$ and $\mathrm{DBN}$ could not be made. Fault evaluation is not performed for this case study since the experiment was not connected to any real building. Thus no real energy impact could be made by the faults.

Table 4-9 Fault diagnostics results for measurement-based AHU-level case study

\begin{tabular}{|c|c|c|c|c|}
\hline Fault Name & $\begin{array}{c}\text { Zhao et. al. } \\
\text { (event-based } \\
\text { BN) }\end{array}$ & $\begin{array}{c}\text { Terminal Fault } \\
\text { Belief } \\
\text { (DBN) }\end{array}$ & $\begin{array}{c}\text { False Negative } \\
\text { Rate } \\
\text { (DBN) }\end{array}$ & $\begin{array}{c}\text { Diagnose } \\
\text { Steps } \\
\text { (DBN) }\end{array}$ \\
\hline$\underline{\text { OA damper stuck close (fully) }}$ & 0.99 & 0.99 & $0 \%$ & 12 \\
\hline$\underline{\text { OA damper stuck close (partial) }}$ & 0.99 & 0.99 & $1.6 \%$ & 26 \\
\hline$\underline{\text { EA damper stuck close (fully) }}$ & No & 0.99 & $0 \%$ & 4 \\
\hline$\underline{\text { EA damper stuck close (partial) }}$ & No & 0.93 & $0.4 \%$ & 13 \\
\hline$\underline{\text { Return fan closed }}$ & 0.99 & 0.99 & $0 \%$ & 1 \\
\hline$\underline{\text { Return fan low speed }}$ & No & 0.99 & $0 \%$ & 1 \\
\hline$\underline{\text { Cooling coil closed (fully) }}$ & 0.93 & 0.99 & $0 \%$ & 1 \\
\hline
\end{tabular}




\begin{tabular}{|c|c|c|c|c|}
\hline Cooling coil closed (partial) & 1.0 & 0.99 & $0 \%$ & 1 \\
\hline$\underline{\text { AHU leak }}$ & No & No & NA & NA \\
\hline
\end{tabular}




\subsubsection{Simulation-based Case Study}
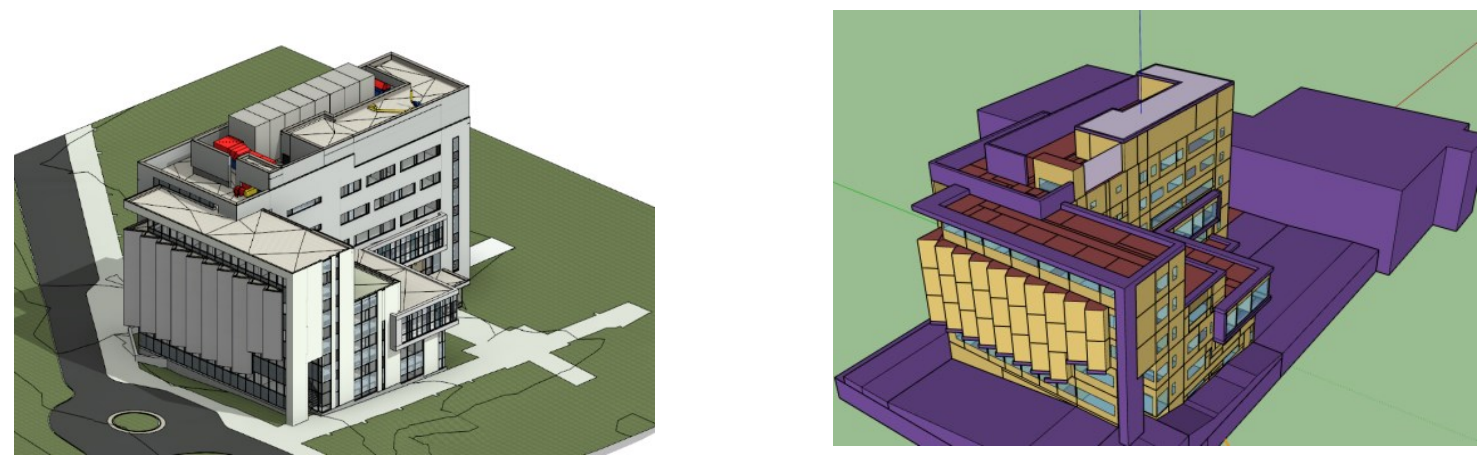

Figure 4-6 overview of the building models. Left: BIM model; right: EnergyPlus model

A simulation-based AHU level case study is performed in addition to the ASHRAE RP-1312 study. The model used is a calibrated EnergyPlus model for the Canal Building on Carleton University campus, same building used for the measurement-based zone level case study. The faults were simulated during both the cooling season as well as the heating season. Akin to the simulation-based zone level case study, faults were simulated by altering inputs inside the EnergyPlus input file, such as boiler efficiency, the schedule for AHU outdoor air intake rate, etc. Likewise, the fault evaluation process was carried out with the same calibrated model following the symptom translation procedure. Below is a list of description of the faults:

1. Boiler low air intake: this fault leads to a lower boiler efficiency for the central hot water system, responsible for supplying domestic hot water and heating water. The efficiency is decreased from 0.89 to 0.6 .

2. AHU supply fan low efficiency: this leads to higher electricity usage to meet the design supply flow rate of the air handling unit. The efficiency of the fan is decreased from 0.8 to 0.5 . 
3. AHU cooling valve stuck closed: this one is analogous to ASHRAE RP-1312, the cooling coil valve is set to stuck closed at $20 \%$ during the cooling season.

4. AHU OA damper stuck closed: also like ASHRAE RP-1312, the outdoor air damper is set to stuck closed at $20 \%$ during the cooling season.

5. Chiller low efficiency: this fault lead to a lower chiller efficiency, causing its DOP to dip from 3.2 to 2.2 , increasing the overall electricity usage.

Table 4-10 summarizes the fault diagnostics results from the AHU simulation study. Overall the results look optimistic with very short detection steps and low false negative rates. The metrics are similar to the previous measurement-based case study, indicating the proposed framework may be quite effective for AHU-level fault detection and diagnostic tasks. As suggested earlier, due to the increased sensing capability in AHU and relatively simplistic parameter estimation and fault-symptom casual relationships for AHU-level case studies, the FDD problem is less complicated than its zone-level counterpart.

Table 4-11 summarizes the fault evaluation results from this simulation study. Showing a similar trend from previous fault evaluation results, the outputs tend to overestimate the actual energy impact. Since the symptoms for all three evaluated faults are not directly observed, this could be the primary cause of the estimation errors. Comfort cannot be evaluated for this AHU-level case study since the feedback control and oversizing of AHU compensated these faults and did not cause an observable dip in thermal comfort on a building level. 
Table 4-10 FDD results for the AHU-level simulation case study

\begin{tabular}{|c|c|c|c|c|}
\hline & Fault Name & $\begin{array}{c}\text { Terminal Fault } \\
\text { Belief } \\
\text { (DBN) }\end{array}$ & $\begin{array}{c}\text { False } \\
\text { Negative } \\
\text { Rate } \\
\text { (DBN) }\end{array}$ & $\begin{array}{c}\text { Detection } \\
\text { Steps } \\
\text { (DBN) }\end{array}$ \\
\hline$\underline{1}$ & $\underline{\text { Boiler low air intake rate }}$ & 0.99 & $1.2 \%$ & 3 \\
\hline$\underline{2}$ & $\underline{\text { AHU supply fan low efficiency }}$ & 0.99 & $0 \%$ & 1 \\
\hline$\underline{3}$ & $\underline{\text { AHU cooling valve stuck closed }}$ & 0.99 & $0 \%$ & 1 \\
\hline$\underline{4}$ & $\underline{\text { AHU OA damper stuck closed }}$ & 0.99 & $0 \%$ & 6 \\
\hline$\underline{5}$ & $\underline{\text { Chiller low efficiency }}$ & 0.99 & $0.8 \%$ & 7 \\
\hline
\end{tabular}

Table 4-11 Fault evaluation results for the AHU-level simulation case study

\begin{tabular}{|c|c|c|c|c|}
\hline \multirow{2}{*}{ Faults } & \multicolumn{2}{|c|}{ Energy (kWh) } & \multicolumn{2}{c|}{ Comfort (PPD) } \\
\cline { 2 - 5 } & Actual* & Evaluated & Actual* & Evaluated \\
\hline$\underline{\text { Boiler low air intake }}$ & 17,660 & 47,838 & N/A & N/A \\
\hline$\underline{\text { AHU supply fan motor deterioration }}$ & 39,803 & $48,286-51,078$ & N/A & N/A \\
\hline Chiller low efficiency & 19,330 & 26,483 & N/A & N/A \\
\hline
\end{tabular}

* simulated with EnergyPlus

\subsection{Summary}

The four cases created in this study demonstrated the effectiveness of the proposed FDDE framework. In general, the results provide a positive outlook with very short diagnostic time, relatively low false negative and false positive rates. Still, some faults take longer to diagnose or have higher false negative rates. When comparing the zone-level and AHU-level studies, the proposed FDDE framework seems more useful towards the AHU system. This is probably because AHU systems are usually well equipped with sensors, making the tasks of extracting more information from the measurements more effective than zone-level tasks. For zone-level case studies, the performance of FDD seems to be bottlenecked by the sensitivity and accuracy of the parameter estimation results. Equipping building systems with more sensors or developing 
better parameter estimation methods may improve the overall effectiveness of the FDDE framework.

As for fault evaluation results, the performance was not as optimal as the fault diagnostic process. The fault evaluation process based on building performance simulation tends to overestimate the impacts caused by faults. More sensor data and better parameter estimates could also make calculating symptom severity more accurate. In addition, some other data-driven fault evaluation methods could be explored. The implications of this FDDE framework and the case studies will be discussed further in the next chapter. 


\section{Chapter: Discussion}

This chapter will summarize the outcomes of this research and discuss the implications and shortcomings of the proposed AFDDE framework. It is then followed by some of the research contributions to related research areas made during this study. Finally, future work including fundamental research questions and improvements specific to the proposed AFDDE framework will be discussed in the last section.

\section{$5.1 \quad$ Research Outcomes}

Overall, results from the case studies using the proposed AFDDE framework show some improvements over the past research. The false positive rates and false negative rates remained low. Overall these rates are below $2 \%$ for most of the fault cases, which means less than two false diagnose each day. The false positive and false negative rates are even lower for AHU due to higher sensor availability at one false alarm every two days. In addition, most of the faults were diagnosed within short amount of time (time steps). Some exceptions do exist, such as an increased phantom load from Section 4.1 which required a long time until adequately diagnosed, and leaking AHU fault from Section 4.2 which could not be properly diagnosed at all. Unfortunately, reporting of false positive rates and false negative rates is not a standard practice in building FDD discipline, so a comparison of similar FDD systems using the same ASHRAE RP-1312 could not be realized. The author recommends that these metrics become more commonly practised within the discipline. Potential methods of improvements for these individual fault cases will be discussed in the following future work section. Another trend discovered from the case studies is that faults with indirectly observed symptoms are generally harder to diagnose. For example, the unintended window opening fault from 4.1 
and 4.1.2 has higher false positive rates and false negative rates. This is somewhat expected since symptoms based on estimated parameters are usually less reliable than the directly observed counterparts due to modelling error. Fundamental research focused on developing more reliable grey-box models and parameter estimation techniques will improve this situation, which will be addressed later.

Although not yet formally tested in a real application, the distributed design (Section 3.1) of the proposed framework would allow different FDDE subtasks such as parameter estimation, symptom generation, fault classification and fault evaluation to be performed on different devices. Compared to putting all these tasks at a central location, this reduces the amount of data needed to be transferred by keeping high frequency calculations at the local level. This means the computationally-demanding fault diagnostic process can be performed on a more powerful server, while more time-sensitive fault detection and symptom generation work can be put on a local controller. This could potentially improve the applicability of the proposed framework, making it more tolerant to congested building automation networks or building energy management networks.

As discussed in Section 2.4.3, the structure of the two-step AFDD system which involves generating symptom first using the fault detection agent then performing diagnostics with the diagnostic agent allows the potential integration of additional AFDD algorithms. The Fault, Symptom and Impact object allow the communication of information between different algorithms and even different AFDD programs to enhance information delivery. For example, an embedded AFDD program on a centrifugal chiller can report its fault diagnoses to the central 
AFDDE system to evaluate its potential impact to the building, enabling potential automated impact mitigation by activating the back-up system.

As demonstrated in Table 4-9, the improvement of the Dynamic Bayesian Network over the conventional Bayesian Network allows the AFDD system to accumulate evidence over time as well as cancelling out sudden false positive diagnoses. Instead of time-stamped alarms of each fault diagnosis, DBN could provide persistent reports on fault status, thus decreasing the overall number of alarms and reduce the false positives being sent to the building operators. As a result, the operators do not have to go through a history of fault alarms. Instead, they will be informed of the current potential faults and their impacts. The operators could also rank the diagnosed faults based on their predefined priorities, or by a quantitative metric such as energy waste and thermal comfort reduction.

Several limitations still exist within the proposed AFDDE framework. The first major limitation is the requirement for manual configuration of the framework, including manual tweaking of the symptom generation, definition of fault-symptom relationships and conditional probabilities. While the relational information is usually transferable between different buildings, this manual setup process still constrains the scalability of the proposed framework. Fortunately, this setup step can be automated, and options to achieve such automation will be discussed.

Another limitation of this work is its reliance on pure passive diagnosis, i.e. the FDDE tasks are performed passively by only accepting measurements from the building systems. To actively seek faults, active diagnosis can be performed by systematically changing control inputs during unoccupied hours and monitor system response. For example, to actively diagnose the performance of an AHU fan, automated tests can be run to monitor the pressure increase/power input ratio at different fan speeds once every two weeks. This way the FDDE system can actively 
search for more data to complement data collected during operation and avoid idling when a building system is off demand. Some research has been done to perform active AFDD, but these works are still quite limited and not yet industrialized [184]. This active diagnosis method is still compatible with the proposed AFDDE framework but requires further expansion of the DBN. The fault evaluation results from the case studies indicate a general trend of overestimating fault impacts. Although overestimation is better than no estimation, it may still make the fault assessments less attractive to the building operators. The fault evaluation method itself, as discussed in Chapter 3, is still at its early stage and need to be further improved. Methods for improving evaluation accuracies will also be discussed in the future work section.

\subsection{Research Contributions}

This thesis has made several novel contributions to not only building system FDD research, but also other disciplines such as intelligent building controls and building performance simulations. This section outlines some of the major contributions from this thesis work:

1. A distributed framework for fault detection, diagnostics and evaluation have been developed. This framework has been published in ASHRAE Transactions [168]. Functional agents such as fault detection agent and fault diagnostic agent have been defined and their tasks assigned. Objects such as symptoms and faults used for communicating between the agents have been established. Fault detection and fault diagnostics have been separated to allow mixed use of different fault detection and diagnosis algorithms. Ways to quantify symptom severity and probability allow the translation of this information to building performance simulation engines to predict the potential impact of the faults. Building operators would have access to more information 
about the faults through quantitative assessments as proposed in this framework. This AFDDE framework is expandable beyond the building energy system domain, and the author believes it is also meaningful to other engineering systems and fault diagnosis problems.

2. A novel algorithm for sequential parameter estimation and state prediction called constrained dual extended Kalman filter was implemented for building systems. More detailed formulation, application, and case studies are being published in Energy and Buildings ( $2^{\text {nd }}$ review). By splitting the parameter vector and state vector into two nonlinear filters, it allows individual tweaking and making them more robust. Its structure also allows the individual filter to be shut down when parameter updates or state predictions are not needed. The addition of inequality constraints to the parameters also eliminates the instability caused by negative parameters inside an additive model. This constrained dual estimation algorithm is applicable not only in Building Engineering discipline but also in other disciplines as well. Moreover, the author hopes it provides an incremental improvement over the existing nonlinear filter algorithms.

3. The usage of Dynamic Bayesian Network for fault diagnosis has been applied in other discipline but is the first time being tested for building systems from this work. The author argues that due to the temporal continuity of most of the building systems responses, a DBN is more suitable to describe the causal relationships between faults and symptoms and how faults continuously affect the system. Even though the implementation of DBN in this research is rudimentary - the introduction of forgetting factor assumes fault probability will be carried over time but is likely to decay when evidence is not further available. As demonstrated in Section 4.2 it already provides an 
improvement over the existing event-based Bayesian Network implementations. What makes the DBN truly shine is the potential of adding fault-symptom relationship across different time steps to better represent cyclical behaviours - self-reinforced cycles caused by faults, which has been previously discussed in Section 3.3. The author suspects this addition will further improve the performance and adaptability of the DBN for fault diagnostics.

4. Conventional AFDD applications use hard evidence, or deterministic symptoms for decision-making. One of the innovations in this research is the adaption of soft evidence, or probabilistic symptoms. In this setup, deterministic symptom values (True or False) can be treated as a special case of a probabilistic symptom ( $99 \%$ True or $99 \%$ False). The way the conventional rule-based symptom generation methods using linear discriminant analysis can still be kept and applied. To make the usage of probabilistic symptoms more realistic, the author has also suggested the use of logistic regression to map symptom severity into symptom probability. Several logistic regression templates are set up for this task, and there is the possibility to automate the creation of logistic regression for each symptom using optimization. The author plans to further expand this ideal and publish an individual paper to advocate the usage of soft evidence for FDD applications.

5. To reduce building performance simulation time and make fault evaluation more responsive to the diagnostics, a novel model reduction method based on selective zone elimination has also been developed. This method has been formulated and generalized in a publication in Journal of Building Performance Simulation [178]. For large-scale simulations, especially BPS models directly translated from BIM models, this model reduction process could potentially decrease the overall simulation time by $70 \%-80 \%$, 
significantly cutting the computation time while keeping the simulation results reasonably reliable. The author is currently working on integrating this model reduction method with the existing building optimization algorithms to significantly reduce BPS optimization time and hoping to make such optimization tasks more suitable to the general design process.

\subsection{Future Work}

While working on this thesis and reviewing previous research, the author realizes some fundamental research topics need to the addressed to help move the current building FDD technology move forward. The following are some of the fundamental questions the author would like to raise to the fellow researchers working on related topics, mainly related to how to decrease the cost to conduct research and improve knowledge exchange between researchers:

1. As of now, there is little guidiance on providing an adequete building operation infrastructure for AFDDE. This includes minimum sensor installations for building systems, functional operation data tagging, and network requirements. While novel technologies such as wireless sensors, printable sensing and the ongoing establishment of new data tagging standard may tackle some aspects of this issue; researchers, industry practioners and stakeholders still need to work together to create a comprehensive guidiance to establishing future-proof infrastructures for advanced AFDDE, prognostics and operation management systems.

2. Unlike other automation and artificial research disciplines, there is a lack of data collaboration and data aggregation efforts. Furthermore, there is an underlying lack of metadata formatting, which is currently being actively discussed among the 
researchers. Some other infrastructure work, such as the standard procedure for data preprocessing is also lacking. All in all, if the collaboration between researchers is enhanced and the free flow of data is created, it will increase the speed of research development, allow the FDD algorithms to be robustly tested, and make the creation of new FDD techniques more cost effective.

3. Research on fault evaluation and information delivery to the stakeholders is still lacking. As reviewed in Chapter 2, only a handful of FDD research has systematically discussed the implication or usage of evaluating fault impacts quantitatively to the building operators. Ideally by providing quantitative fault assessment will help building operators prioritize their work and making FDD systems more impactful. However, the validity of this argument is not thoroughly surveyed from the industry. A survey-based study would undoubtedly help the researchers better understand the need for such processes. Also, there is a lack of a general framework for evaluating faults using different models discussed in Section 2.5, the author is currently working on developing such framework [185] and getting feedback from the industry and will be part of his future work.

4. There has been little consensus of using fault positive rate (FPR), false negative rate (FNR) and other metrics such as precision and recall for evaluating AFDD algorithms for buildings. Ideally the importance of FPR and FNR are unequal for different kinds of faults. For example, a critical fault related to safety requires an ideal zero FNR while minimizing FPR, for a less critical fault a zero FPR is preferred to reduce false alarms. In addition, there is little investigation of how to customize cost/loss functions to optimize FNR/FPR differently during AFDD model training. More discussion on 
this topic will make AFDD research more practical and will provide a standard platform to compare different AFDD algorithms.

Besides fundamental questions, there are several ways to expand upon the proposed AFDDE framework to further improve and test its usability. The priority is to add self-learning capabilities to the DBN for better fault diagnostics and self-tuning functions to logistic regressions for improved symptom generation. As mentioned previously, the current manual setup process is not very scalable, and could not be updated quickly. Instead, interactive learning based on operator's feedback could be used. For example, if a building operator reported a false positive diagnosis, the conditional probabilities for this fault inside the DBN would be recalibrated recursively using previous data to reduce false positive rate. On the other hand, if a building operator indicated the AFDDE system failed to acknowledge a fault or a symptom, the AFDDE system will then first update the symptom's logistic regression to make it more sensitive, then retrain the DBN to successfully diagnose this fault. Eventually, this process will also make the AFDDE system adapt to the priorities of the building operators since most likely the most critical faults will be examined for false negatives, and the most inaccurate diagnosis will be flagged as false positives.

Another potential future work is to integrate some automated model calibration techniques for building performance simulation models to improve the applicability of fault evaluation process, such as meta-model fitting [172]. To further enhance the practically of the fault evaluation process, some simple grey-box models could be applied to evaluate the faults' impact on firstorder system response. Examples include using data-driven simple supply air fan efficiency models to evaluate the impact of a lowered efficiency symptom. 
Hopefully, this AFDDE framework could be implemented inside a real building automation system or building energy management system for prototyping. If a testing bed capable of emulating faults is available, more fault cases can be tested to expand the number of faults inside the existing DBN. Ideally, evidence apart from sensor measurements and estimated parameters could also be incorporated - information such as date, time and observations from the occupants/operators could also be used for fault diagnostics. And finally, this AFDDE framework may be incorporated beyond the energy spectrum, as it is possible to include other operation faults such as broken automatic doors, broken elevators, to make this system more comprehensive.

To summarize, the proposed AFDDE framework showed promise but still is in its early stage. Numerous potential future extensions could make it even more powerful and user-friendly. Some fundamental questions, however, still need to be pursued by researchers to promote automated fault detection, diagnostics, and evaluation technology inside built environment to bring even more meaningful benefits to the society. 


\section{Chapter: Conclusions}

This thesis proposed a novel distributed fault detection, diagnostics and evaluation framework for building systems. The goal of this research was to improve upon the current fault detection and diagnostics methodology while providing quantitative evaluations to the building operators for better decision-making. Before establishing the methodology, a review of building FDD research over the last two decades was conducted in Chapter 2. From this review, priority research problems and best potential options to tackle these problems were summarized. Fault and symptom in this framework are based on probabilistic representation, and the severity of symptom is quantified for fault evaluation purpose. Three types of functioning agents, including algorithms employed inside them, are detailed in Chapter 3. Besides establishing the framework in Chapter 3, the author of this thesis also tried to generalize some of its components to other disciplines.

Many of the components inside the proposed FDDE framework, such as parameter estimation and model reduction, have been published as independent research with publicly available code in Python and R. These contributions and their related articles are listed below:

1. Establishment of a distributed FDDE framework using probabilistic description of faults and symptoms. The distributed design allows the framework to be implemented inside an existing building automation network, or at a central server. The probabilistic description also enables the framework to incorporate uncertainties such as measurement error, modelling error and knowledge limitations. This architecture increases the reliability and usability of the framework overall and can be used beyond building systems. This framework will be published in the upcoming ASHRAE transaction titled as: 
Development of a distributed fault detection, diagnostics and evaluation system, ASHRAE Transactions, 2018 [168]

2. Formulation of constrained dual Extended Kalman Filter for parameter estimation, state prediction and anomaly detection. The usage of dual filters increases the algorithm efficiency, and the inclusion of inequality constraints enhances the overall numerical stability. This contribution is not only useful to FDD applications, but also applicable to model predictive control research. An upcoming paper is under review titled Sequential State Prediction and Parameter Estimation with Constrained Dual Extended Kalman Filter for Building Zone Thermal Responses, Energy and Buildings, article under review; a previous conference paper has also been published as Building Fault Detection with Kalman Filter Based Methods, eSim 2016, recipient of the best paper award [69].

3. Creation of a building energy model reduction method based on selective zone elimination. As demonstrated from previous study, this model reduction method can reduce simulation time by up to $95 \%$. It can also be used for building energy optimization purposes with a computation reduction by approximately $70 \%$ for a large scale building projects. Published articles: Building Energy Model Reduction using Model-ClusterReduce Pipeline, Journal of Building Performance Simulation, December 2017 [178]; Building Energy Model Reduction using Principal Component Analysis and Affinity Propagation Clustering of Thermal Zones, ASIM 2016, recipient of the best paper award [180].

4. Usage of building performance simulation for fault evaluation and a standard procedure of translating symptoms to BPS inputs, related article: Using Building Performance Simulation for Fault Impact Evaluation, eSim 2018 [185]. This area of research of using 
BPS for fault evaluation and fault management is still very new and will be further expanded in the future. Another potential method of proving systematic fault evaluation on building systems is through the usage of data driven approach. However, this requires much more data collaboration effort, which is currently being discussed actively by the building AFDD community.

Implementations of the proposed AFDDE methodology and four different case studies at zonelevel and AHU-level were presented in Chapter 4. Overall the proposed implementations achieved both low false positive rate and low false positive rate. Although the fault evaluation process tends to overestimate the actual fault impacts, they still provided extra insight to assess different faults. In addition, the selection of different time steps did not visibly affect the outcomes, indicating the proposed fault detection and diagnostics methods could be very flexible for different applications. However, one major bottleneck came from the case studies is the framework's reliance on parameter estimation results since many symptoms are not directly observable from the sensors. More responsive parameter estimation techniques can greatly reduce the amount of time steps required to properly diagnose several faults studied in Chapter 4, such as unintended window opening fault and unwanted phantom load.

There are plenty of potential improvements to the proposed framework, such as taking advantage of machine learning to automatically configure the AFDDE system and continuously improve its performance by taking feedback from the operators. In addition, many fundamental research topics arise from this research, such as adopting a standard metadata format for building data among the researchers, as well as mandating minimum sensing requirements for new constructions and retrofits. These fundamental research topics and future work were detailed in Chapter 5. The author of this thesis hopes this work may become useful not only for its specific 
application but also meaningful to engineering research at large. Little by little our work could help in making this world a better place. 


\section{Appendices}

\section{Appendix A Table of reviewed articles}

\begin{tabular}{|c|c|c|c|c|c|c|c|c|}
\hline Topic & Author & Year & $\begin{array}{l}\text { Building } \\
\text { System }\end{array}$ & $\begin{array}{l}\text { Fault Detection } \\
\text { Method }\end{array}$ & $\begin{array}{l}\text { Fault Diagnostics } \\
\text { Method }\end{array}$ & $\begin{array}{l}\text { Separate } \\
\text { FDD? }\end{array}$ & $\begin{array}{l}\text { Actual } \\
\text { Data }\end{array}$ & $\begin{array}{l}\text { Simulated } \\
\text { Data }\end{array}$ \\
\hline$F D D$ & Zhao et al & 2017 & $\mathrm{AHU}$ & Rules & $\begin{array}{l}\text { Bayesian } \\
\text { Network }\end{array}$ & Yes & Yes & No \\
\hline$F D D$ & Verbert et al & 2017 & HVAC & Grey-box Model & $\begin{array}{l}\text { Bayesian } \\
\text { Network }\end{array}$ & Yes & No & Yes \\
\hline$F D$ & Every et al & 2017 & HVAC & $\begin{array}{l}\text { SVM and } \\
\text { Gaussian } \\
\text { Regressor }\end{array}$ & NA & $\begin{array}{l}\text { Detection } \\
\text { Only }\end{array}$ & Yes & No \\
\hline$F D$ & Wook & 2017 & $\begin{array}{l}\text { Whole } \\
\text { Building }\end{array}$ & Black-box Model & NA & $\begin{array}{l}\text { Detection } \\
\text { Only }\end{array}$ & No & Yes \\
\hline$F D D$ & Yan et al & 2017 & $\mathrm{AHU}$ & Grey-box Model & Rules & Yes & No & Yes \\
\hline$F D$ & Yan et al & 2017 & Chiller & Grey-box Model & NA & $\begin{array}{l}\text { Detection } \\
\text { Only }\end{array}$ & Yes & No \\
\hline$F D D$ & $\begin{array}{l}\text { Sharifi and } \\
\text { Langari }\end{array}$ & 2017 & Sensor & Black-box Model & $\begin{array}{l}\text { GMM (black-box } \\
\text { model) }\end{array}$ & Yes & No & Yes \\
\hline$F D D$ & Yan et al & 2017 & $\mathrm{AHU}$ & NA & ELM & No & No & Yes \\
\hline$F D D$ & Wang et al & 2017 & Chiller & NA & $\begin{array}{l}\text { Conditional } \\
\text { Bayesian } \\
\text { Network }\end{array}$ & No & Yes & No \\
\hline$F D D$ & Guo et al & 2017 & VRF & NA & BPNN & No & Yes & No \\
\hline$F D$ & $\begin{array}{l}\text { Horrigan et } \\
\text { al }\end{array}$ & 2017 & $\begin{array}{l}\text { Whole } \\
\text { Building }\end{array}$ & $\begin{array}{l}\text { EWMA and } \\
\text { Stewards Charts }\end{array}$ & NA & $\mathrm{Na}$ & Yes & No \\
\hline$F D D$ & Chang et al & 2017 & $\begin{array}{l}\text { HVAC } \\
\text { system }\end{array}$ & NA & $\begin{array}{l}\text { General Machine } \\
\text { Learning }\end{array}$ & No & No & Yes \\
\hline$F D$ & $\begin{array}{l}\text { Chen and } \\
\text { Wen }\end{array}$ & 2017 & $\begin{array}{l}\text { Whole } \\
\text { Building }\end{array}$ & PCA & NA & NA & Yes & No \\
\hline$F D$ & Turner et al & 2017 & HVAC & ARX & NA & NA & No & Yes \\
\hline$F D D$ & Gao et al & 2016 & $\begin{array}{l}\text { HVAC } \\
\text { Systems }\end{array}$ & Grey-box Model & Rules & Yes & No & Yes \\
\hline$F D D$ & Li et al & 2016 & $\begin{array}{l}\text { Cooling } \\
\text { system }\end{array}$ & NA & Classifier & No & Yes & No \\
\hline$F D D$ & Li et al & 2016 & Chiller & NA & $\begin{array}{l}\text { Support Vector } \\
\text { Data Description }\end{array}$ & No & Yes & No \\
\hline$F D D$ & Beghi et al & 2016 & Chiller & PCA & $\begin{array}{l}\text { reconstruction- } \\
\text { based }\end{array}$ & Yes & Yes & No \\
\hline$F D D$ & Wang et al & 2016 & $\begin{array}{l}\text { Whole } \\
\text { Building }\end{array}$ & Grey-box model & Rules & Yes & No & No \\
\hline FDD & He et al & 2016 & Chiller & NA & $\begin{array}{l}\text { Bayesian } \\
\text { Network }\end{array}$ & No & Yes & No \\
\hline$F D D$ & Yan et al & 2016 & $\mathrm{AHU}$ & NA & $\begin{array}{l}\text { Classification } \\
\text { Tree (CART) }\end{array}$ & No & Yes & No \\
\hline$F D D$ & Li et al & 2016 & Chiller & NA & $\begin{array}{l}\text { Linear } \\
\text { discriminant } \\
\text { analysis }\end{array}$ & No & Yes & No \\
\hline$F D D$ & Cotrufo & 2016 & Chiller & PCA & $\begin{array}{l}\text { variable } \\
\text { identification }\end{array}$ & Yes & Yes & No \\
\hline$F D$ & Peña & 2016 & $\begin{array}{l}\text { Whole } \\
\text { Building }\end{array}$ & Rules & NA & NA & Yes & No \\
\hline$F D D$ & Wang et al & 2016 & $\mathrm{AHU}$ & EWMA & Rules & Yes & Yes & No \\
\hline
\end{tabular}




\begin{tabular}{|c|c|c|c|c|c|c|c|c|}
\hline$F D$ & Arya et al & 2016 & $\begin{array}{l}\text { Whole } \\
\text { Building }\end{array}$ & Black-box Model & NA & NA & Yes & No \\
\hline$F D D$ & $\begin{array}{l}\text { Dey and } \\
\text { Dong }\end{array}$ & 2016 & $\mathrm{AHU}$ & Rules & $\begin{array}{l}\text { Bayesian } \\
\text { Network }\end{array}$ & Yes & Yes & No \\
\hline$F D D$ & $\begin{array}{l}\text { Yunguang } \\
\text { Gao et al }\end{array}$ & 2016 & $\begin{array}{l}\text { Dehumidifie } \\
r\end{array}$ & NARX & Logic & Yes & No & Yes \\
\hline$F D D$ & $\begin{array}{l}\text { A. Capozzoli } \\
\text { et al }\end{array}$ & 2015 & $\begin{array}{l}\text { Lighting } \\
\text { System }\end{array}$ & ANN & No & $\begin{array}{l}\text { Detection } \\
\text { Only }\end{array}$ & Yes & No \\
\hline$F D D$ & $\begin{array}{l}\text { Christian } \\
\text { Jones }\end{array}$ & 2015 & $\begin{array}{l}\text { HVAC } \\
\text { subsystems }\end{array}$ & NA & $\begin{array}{l}\text { LAPART } \\
\text { Algorithm }\end{array}$ & No & Yes & Yes \\
\hline$F D$ & Dawei He & 2015 & HVAC & Grey-box Model & NA & $\begin{array}{l}\text { Detection } \\
\text { Only }\end{array}$ & NA & NA \\
\hline$F D$ & $\begin{array}{l}\text { Guanjing Lin } \\
\text { et al }\end{array}$ & 2015 & $\begin{array}{l}\text { Whole } \\
\text { Building }\end{array}$ & Grey-box Model & NA & $\begin{array}{l}\text { Detection } \\
\text { Only }\end{array}$ & NA & NA \\
\hline$F D D$ & $\begin{array}{l}\text { Mulumba et } \\
\text { al. }\end{array}$ & 2015 & $\mathrm{AHU}$ & ARX Model & SVM & Yes & Yes & Yes \\
\hline$F D D$ & Padilla et al. & 2015 & $\mathrm{AHU}$ & PCA & $\begin{array}{l}\text { Active Functional } \\
\text { Testing }\end{array}$ & Yes & Yes & Yes \\
\hline$F D$ & $\begin{array}{l}\text { M. Petit- } \\
\text { Pierre }\end{array}$ & 2015 & $\begin{array}{l}\text { Building } \\
\text { Energy }\end{array}$ & KPCA & NA & No & Yes & No \\
\hline$F D D$ & Tran et al & 2015 & Chiller & Statistical Model & Logic & Yes & Yes & No \\
\hline $\begin{array}{r}\text { FD, } \\
\text { Contr } \\
\text { ol }\end{array}$ & $\begin{array}{l}\text { Noor } \\
\text { Sulaiman et } \\
\text { al. }\end{array}$ & 2015 & $\begin{array}{l}\text { Chilled } \\
\text { Water } \\
\text { System }\end{array}$ & Fuzzy Logic & NA & $\begin{array}{l}\text { Detection } \\
\text { Only }\end{array}$ & No & Yes \\
\hline$F D D$ & $\begin{array}{l}\text { Yang Zhao } \\
\text { et al }\end{array}$ & 2015 & $\begin{array}{l}\text { Coils and } \\
\text { Sensors }\end{array}$ & Rules & $\begin{array}{l}\text { Bayesian } \\
\text { Network }\end{array}$ & Yes & Yes & Yes \\
\hline$F D D$ & $\begin{array}{l}\text { Katipamula } \\
\text { et al }\end{array}$ & 2015 & $\begin{array}{l}\text { Rooftop } \\
\text { Units }\end{array}$ & Rules & Logic & Yes & Yes & No \\
\hline$F D D$ & Bovini et al & 2014 & Chiller & Grey-box Model & $\begin{array}{l}\text { Parameter to } \\
\text { components }\end{array}$ & Yes & No & Yes \\
\hline$F D D$ & Bruton et al & 2014 & $\mathrm{AHU}$ & Rules & Logic & Yes & Yes & No \\
\hline$F D$ & Burgas et al & 2014 & $\begin{array}{l}\text { Building } \\
\text { Energy }\end{array}$ & PCA & NA & No & Yes & No \\
\hline$F D D$ & $\begin{array}{l}\text { Baoping Cai } \\
\text { et al }\end{array}$ & 2014 & GSHP & $\begin{array}{l}\text { Bayesian } \\
\text { network }\end{array}$ & $\begin{array}{l}\text { Bayesian } \\
\text { Network }\end{array}$ & Both & No & Yes \\
\hline$F D$ & $\begin{array}{l}\text { Zhimin Du } \\
\text { et al }\end{array}$ & 2014 & Sensors & ANN and BPNN & NA & No & No & Yes \\
\hline$F D$ & $\begin{array}{l}\text { Golparvar- } \\
\text { Fard et al }\end{array}$ & 2014 & $\begin{array}{l}\text { Building } \\
\text { Envelope }\end{array}$ & First principle & NA & No & Yes & No \\
\hline$F D$ & $\begin{array}{l}\text { J. Leloux et } \\
\text { al }\end{array}$ & 2014 & BIPV & First principle & NA & No & Yes & No \\
\hline$F D$ & I. Khan et al & 2014 & $\begin{array}{l}\text { Lighting } \\
\text { System }\end{array}$ & ANN & NA & No & Yes & No \\
\hline$F D D$ & $\begin{array}{l}\text { Kocyigit et } \\
\text { al }\end{array}$ & 2014 & Chiller & First principle & Manual & Yes & Yes & No \\
\hline$F D D$ & Lauro et al & 2014 & Fan Coils & Peak detection & fuzzy logic & Yes & Yes & No \\
\hline$F D$ & S Li et al. & 2014 & $\mathrm{AHU}$ & Statistical Model & NA & $\begin{array}{l}\text { Detection } \\
\text { Only }\end{array}$ & Yes & No \\
\hline$F D D$ & $\begin{array}{l}\text { F Marino et } \\
\text { al }\end{array}$ & 2014 & $\begin{array}{l}\text { Lighting } \\
\text { System }\end{array}$ & Peak detection & fuzzy logic & Yes & Yes & No \\
\hline$F D D$ & $\begin{array}{l}\text { Mulumba et } \\
\text { al }\end{array}$ & 2014 & HVAC & $\begin{array}{l}\text { Inverse Grey-box } \\
\text { Model }\end{array}$ & Rules & Yes & No & Yes \\
\hline$F D D$ & $\begin{array}{l}\text { B } \\
\text { Narayanasw } \\
\text { amy et al }\end{array}$ & 2014 & $\begin{array}{l}\text { VAV } \\
\text { Terminals }\end{array}$ & Clustering & NA & $\begin{array}{l}\text { Detection } \\
\text { Only }\end{array}$ & Yes & No \\
\hline$F D D$ & $\begin{array}{l}\text { R. Sterling } \\
\text { et al }\end{array}$ & 2014 & $\mathrm{AHU}$ & $\begin{array}{l}\text { Grey-box and } \\
\text { Qualitative }\end{array}$ & NA & No & NA & NA \\
\hline
\end{tabular}




\begin{tabular}{|c|c|c|c|c|c|c|c|c|}
\hline$F D$ & $\begin{array}{l}\text { Biao Sun et } \\
\text { al }\end{array}$ & 2014 & $\begin{array}{l}\text { Chiller, } \\
\text { Cooling } \\
\text { Tower }\end{array}$ & $\begin{array}{l}\text { SPC w. Kalman } \\
\text { Filter }\end{array}$ & NA & No & Yes & No \\
\hline$F D$ & $\begin{array}{l}\text { Wijayasekar } \\
\text { a et al }\end{array}$ & 2014 & HVAC & Clustering & NA & No & Yes & No \\
\hline$F D D$ & Yan Ke et al & 2014 & Chiller & ARX & SVM & Yes & Yes & No \\
\hline$F D D$ & $\begin{array}{l}\text { Zhimin Du } \\
\text { et al }\end{array}$ & 2013 & HVAC & NN and NPNN & $\begin{array}{l}\text { Subtractive } \\
\text { clustering }\end{array}$ & Yes & No & Yes \\
\hline$F D$ & $\begin{array}{l}\text { Ploennigs } \\
\text { and Chen }\end{array}$ & 2013 & $\begin{array}{l}\text { Whole } \\
\text { Building }\end{array}$ & ARMA & $\begin{array}{l}\text { Generalized } \\
\text { Additive Model }\end{array}$ & Yes & Yes & No \\
\hline$F D D$ & Khan et al & 2013 & $\begin{array}{l}\text { Whole } \\
\text { Building }\end{array}$ & $\begin{array}{l}\text { CART and } \\
\text { Clustering }\end{array}$ & NA & $\begin{array}{l}\text { Detection } \\
\text { Only }\end{array}$ & Yes & No \\
\hline$F D$ & $\begin{array}{l}\text { Fontugne et } \\
\text { al }\end{array}$ & 2013 & $\begin{array}{l}\text { Whole } \\
\text { Building }\end{array}$ & $\begin{array}{l}\text { Strip, Bind and } \\
\text { Search }\end{array}$ & NA & $\begin{array}{l}\text { Detection } \\
\text { Only }\end{array}$ & Yes & No \\
\hline$F D$ & Yang et al & 2013 & $\mathrm{AHU}$ & $\begin{array}{l}\text { Fractal } \\
\text { Correlation } \\
\text { Dimension }\end{array}$ & NA & $\begin{array}{l}\text { Detection } \\
\text { Only }\end{array}$ & No & Yes \\
\hline$F D D$ & $\begin{array}{l}\text { Magoules et } \\
\text { al }\end{array}$ & 2013 & $\begin{array}{l}\text { Whole } \\
\text { Building }\end{array}$ & NA & $\begin{array}{l}\text { RDP Neural } \\
\text { Network }\end{array}$ & No & No & Yes \\
\hline$F D D$ & Zhao et al & 2013 & Chiller & Rules & $\begin{array}{l}\text { Bayesian } \\
\text { Network }\end{array}$ & Yes & Yes & No \\
\hline$F D$ & Zhao et al & 2013 & Chiller & $\begin{array}{l}\text { Support Vector } \\
\text { Description }\end{array}$ & NA & $\begin{array}{l}\text { Detection } \\
\text { Only }\end{array}$ & Yes & No \\
\hline$F D D$ & $\begin{array}{l}\text { Zaheeruddin } \\
\text { and } \\
\text { Tudoroiu }\end{array}$ & 2012 & HVAC & Grey-box Model & Rules & Yes & No & Yes \\
\hline$F D D$ & $\begin{array}{l}\text { Parvaresh et } \\
\text { al }\end{array}$ & 2012 & HVAC & NA & $\begin{array}{l}\text { T-S fuzzy } \\
\text { classifier }\end{array}$ & No & No & Yes \\
\hline$F D$ & Pang et al & 2012 & $\begin{array}{l}\text { Whole } \\
\text { Building }\end{array}$ & First principle & NA & No & Yes & No \\
\hline$F D D$ & Zhu et al & 2012 & Sensors & NA & ANN & No & No & Yes \\
\hline$F D$ & Guo et al & 2012 & HVAC & $\begin{array}{l}\text { Support vector } \\
\text { machine }\end{array}$ & NA & $\begin{array}{l}\text { Detection } \\
\text { Only }\end{array}$ & No & No \\
\hline$F D$ & Hu et al & 2012 & Chiller & PCA & NA & $\begin{array}{l}\text { Detection } \\
\text { Only }\end{array}$ & Yes & No \\
\hline$F D D$ & Wang et al. & 2012 & $\mathrm{AHU}$ & Grey-box Model & Rules & Yes & Yes & No \\
\hline$F D D$ & Wang et al. & 2012 & $\begin{array}{l}\text { VAV } \\
\text { Terminals }\end{array}$ & Grey-box Model & Rules & Yes & Yes & No \\
\hline$F D D$ & $\begin{array}{l}\text { Zimmerman } \\
\text { et al }\end{array}$ & 2012 & HVAC & First principle & Rules & Yes & No & Yes \\
\hline$F D D$ & $\begin{array}{l}\text { Thumati et } \\
\text { al }\end{array}$ & 2011 & HVAC & Grey-box Model & Observer & Yes & No & Yes \\
\hline$F D D$ & Dehestani & 2011 & HVAC & NA & SVM & No & No & Yes \\
\hline$F D D$ & Xiao et al & 2011 & Chiller & Grey-box Model & Rules & Yes & Yes & No \\
\hline$F D$ & Yang et al & 2011 & Sensor & $\begin{array}{l}\text { Fractal } \\
\text { Correlation } \\
\text { Dimension }\end{array}$ & NA & NA & No & Yes \\
\hline$F D D$ & Wang et al & 2011 & $\begin{array}{l}\text { VAV } \\
\text { Terminals }\end{array}$ & Grey-box Model & Rules & Yes & Yes & No \\
\hline$F D D$ & West et al & 2011 & HVAC & NA & Black-box Model & No & Yes & No \\
\hline$F D D$ & Wang et al & 2010 & Sensors & PCA & Black-box Model & No & No & Yes \\
\hline$F D$ & Wu and Sun & 2010 & HVAC & PCA & NA & $\begin{array}{l}\text { Detection } \\
\text { Only }\end{array}$ & Yes & No \\
\hline$F D D$ & Sun et al & 2010 & Chiller & Grey-box Model & Rules & Yes & No & Yes \\
\hline$F D D$ & $\begin{array}{l}\text { Sakthivel et } \\
\text { al }\end{array}$ & 2010 & Chiller & NA & CART & No & Yes & No \\
\hline
\end{tabular}




\begin{tabular}{|c|c|c|c|c|c|c|c|}
\hline$F D D$ & Fan et al & 2010 & $\mathrm{AHU}$ & NA & ANN & No & No \\
\hline$F D$ & Jacob et al & 2010 & $\begin{array}{l}\text { Whole } \\
\text { Building }\end{array}$ & $\begin{array}{l}\text { Clustering and } \\
\text { Regression }\end{array}$ & NA & $\begin{array}{l}\text { Detection } \\
\text { Only }\end{array}$ & No \\
\hline$F D D$ & Han et al & 2010 & Chiller & PCA & SVM & Yes & Yes \\
\hline$F D D$ & $\begin{array}{l}\text { Najafi, } \\
\text { Massieh }\end{array}$ & 2010 & $\mathrm{AHU}$ & NA & $\begin{array}{l}\text { Bayesian } \\
\text { Network }\end{array}$ & No & Mo \\
\hline$F D D$ & $\begin{array}{l}\text { Zhimin Du } \\
\text { et al }\end{array}$ & 2009 & Sensors & Signal Analysis & $\begin{array}{l}\text { ANN } \\
\text { Classification }\end{array}$ & Yes & Mo \\
\hline$F D$ & $\begin{array}{l}\text { Chen and } \\
\text { Lan }\end{array}$ & 2009 & Chiller & PCA & NA & $\begin{array}{l}\text { Detection } \\
\text { Only }\end{array}$ & Yes \\
\hline$F D D$ & Du et al & 2009 & Sensors & PCA & PCA & No & No \\
\hline$F D D$ & Zhou et al & 2009 & Chiller & Grey-box Model & ANN & Yes & No \\
\hline$F D D$ & $\begin{array}{l}\text { Holcomb et } \\
\text { al }\end{array}$ & 2009 & $\begin{array}{l}\text { Whole } \\
\text { Building }\end{array}$ & NA & Black-box Model & No & No \\
\hline$F D D$ & $\begin{array}{l}\text { Tudoroiu et } \\
\text { al }\end{array}$ & 2008 & HVAC & UKF & Rules & Yes & No \\
\hline$F D$ & Yang et al & 2008 & Sensors & Grey-box Model & Rules & Yes & Yes \\
\hline$F D D$ & Du et al & 2008 & $\begin{array}{l}\text { VAV } \\
\text { Terminals }\end{array}$ & PCA & Joint Angle Plot & Yes & Mo \\
\hline$F D D$ & Xu et al & 2008 & Sensors & PCA & Rules & Yes & No \\
\hline$F D D$ & Kim et al & 2008 & $A C$ & Grey-box Model & Rules & No & Yes \\
\hline$F D D$ & Song et al & 2008 & HVAC & Grey-box Model & Rules & Yes & No \\
\hline$F D D$ & Du et al & 2007 & Sensors & PCA & Joint Angle Plot & Yes & No \\
\hline$F D$ & Lalot et al & 2007 & $\begin{array}{l}\text { Heat } \\
\text { Exchanger }\end{array}$ & $\begin{array}{l}\text { PCA Grey-box } \\
\text { Model }\end{array}$ & NA & $\begin{array}{l}\text { Detection } \\
\text { Only }\end{array}$ & Yes \\
\hline$F D D$ & $\begin{array}{l}\text { Namburu et } \\
\text { al }\end{array}$ & 2007 & Chiller & NA & Black-box Model & No & Yes \\
\hline$F D$ & Lo et al & 2007 & $\mathrm{AHU}$ & Fuzzy Logic & NA & $\begin{array}{l}\text { Detection } \\
\text { Only }\end{array}$ & No \\
\hline$F D D$ & $\begin{array}{l}\text { Liang and } \\
\text { Du }\end{array}$ & 2007 & HVAC & NA & $\begin{array}{l}\text { Support Vector } \\
\text { Machine }\end{array}$ & No & No \\
\hline$F D D$ & $\begin{array}{l}\text { Wang and } \\
\text { Xiao }\end{array}$ & 2006 & Sensors & PCA & Rules & Yes & No \\
\hline$F D$ & Schein et al & 2006 & $\mathrm{AHU}$ & Rules & NA & $\begin{array}{l}\text { Detection } \\
\text { Only }\end{array}$ & No \\
\hline$F D D$ & Hou et al & 2006 & Sensors & Rules & ANN & Yes & Yes \\
\hline$F D D$ & $\begin{array}{l}\text { Wang and } \\
\text { Cui }\end{array}$ & 2006 & Chiller & PCA & Rules & Yes & Yes \\
\hline$F D D$ & Zogg et al & 2006 & Chiller & Grey-box Model & Clustering & Yes & Yes \\
\hline$F D D$ & $\begin{array}{l}\text { Cui and } \\
\text { Wang }\end{array}$ & 2005 & Chiller & First principle & Rules & Yes & Yes \\
\hline$F D D$ & $\begin{array}{l}\text { Tudoroiu et } \\
\text { al }\end{array}$ & 2005 & Valve & $\begin{array}{l}\text { Frequency } \\
\text { Analysis }\end{array}$ & Rules & Yes & Yes \\
\hline$F D D$ & Cho et al & 2005 & HVAC & Grey-box Model & Rules & Yes & Yes \\
\hline$F D D$ & $\begin{array}{l}\text { Tassou and } \\
\text { Grace }\end{array}$ & 2005 & Chiller & Rules & Rules & Yes & Yes \\
\hline$F D D$ & $\begin{array}{l}\text { Qin and } \\
\text { Wang }\end{array}$ & 2005 & HVAC & PCA & Rules & Yes & No \\
\hline$F D D$ & Lee et al & 2004 & $\mathrm{AHU}$ & Black-box Model & Rules & Yes & No \\
\hline$F D D$ & $\begin{array}{l}\text { Wang and } \\
\text { Zhang }\end{array}$ & 2004 & Valve & Black-box Model & Rules & Yes & No \\
\hline$F D$ & Armstrong & 2004 & HVAC & Grey-box Model & Rules & Yes & Yes \\
\hline
\end{tabular}




\begin{tabular}{|c|c|c|c|c|c|c|c|c|}
\hline$F D$ & $\begin{array}{l}\text { Pakanen } \\
\text { and } \\
\text { Sundquist }\end{array}$ & 2003 & $\mathrm{AHU}$ & Grey-box Model & Rules & Yes & Yes & No \\
\hline$F D D$ & $\begin{array}{l}\text { Bailey and } \\
\text { Kreider }\end{array}$ & 2003 & Chiller & NA & ANN & No & Yes & No \\
\hline$F D$ & Song et al & 2003 & $\begin{array}{l}\text { Whole } \\
\text { Building }\end{array}$ & First principle & NA & $\begin{array}{l}\text { Detection } \\
\text { Only }\end{array}$ & Yes & No \\
\hline$F D D$ & Yu et al & 2003 & Radiator & First principle & Rules & Yes & No & Yes \\
\hline$F D D$ & $\begin{array}{l}\text { Wang and } \\
\text { Wang }\end{array}$ & 2002 & HVAC & Grey-box Model & Rules & Yes & No & Yes \\
\hline$F D D$ & Yu et al & 2002 & HVAC & First principle & Rules & Yes & No & Yes \\
\hline$F D D$ & Shaw et al & 2002 & HVAC & Grey-box Model & Rules & Yes & Yes & No \\
\hline$F D D$ & Jia & 2002 & Chiller & First principle & Rules & Yes & Yes & No \\
\hline$F D D$ & $\mathrm{Li}$ and Braun & 2002 & HVAC & Black-box Model & Rules & Yes & Yes & No \\
\hline$F D D$ & Luo & 2001 & HVAC & Grey-box Model & Rules & Yes & Yes & No \\
\hline$F D D$ & Ahn et al & 2001 & $\begin{array}{l}\text { Cooling } \\
\text { Towers }\end{array}$ & Grey-box Model & Rules & Yes & & \\
\hline$F D D$ & Kumar et al & 2001 & $\mathrm{AHU}$ & Grey-box Model & Rules & Yes & Yes & No \\
\hline$F D D$ & $\begin{array}{l}\text { Dexter and } \\
\text { Ngo }\end{array}$ & 2001 & $A C$ & First principle & fuzzy logic & No & No & Yes \\
\hline$F D D$ & Yoshida et al & 2001 & $\mathrm{AHU}$ & Grey-box Model & Rules & Yes & Yes & No \\
\hline$F D D$ & $\begin{array}{l}\text { Chen and } \\
\text { Braun }\end{array}$ & 2001 & $A C$ & Grey-box Model & Rules & Yes & Yes & No \\
\hline$F D$ & House et al & 2001 & $\mathrm{AHU}$ & Rules & NA & $\begin{array}{l}\text { Detection } \\
\text { Only }\end{array}$ & Yes & No \\
\hline$F D D$ & $\begin{array}{l}\text { Yoshida and } \\
\text { Kumar }\end{array}$ & 1999 & $\mathrm{AHU}$ & Grey-box Model & Rules & Yes & Yes & No \\
\hline$F D D$ & $\begin{array}{l}\text { Wang and } \\
\text { Wang }\end{array}$ & 1999 & $A C$ & Grey-box Model & Rules & Yes & No & Yes \\
\hline$F D D$ & $\begin{array}{l}\text { Katipamula } \\
\text { et al. }\end{array}$ & 1999 & Economizer & Grey-box Model & Rules & Yes & Yes & No \\
\hline$F D$ & $\begin{array}{l}\text { Morisot and } \\
\text { Marchio }\end{array}$ & 1999 & $\mathrm{AHU}$ & Black-box Model & NA & $\begin{array}{l}\text { Detection } \\
\text { Only }\end{array}$ & No & Yes \\
\hline$F D D$ & Dodier et al & 1998 & HVAC & Grey-box Model & Rules & Yes & No & Yes \\
\hline$F D D$ & Piette et al & 1998 & HVAC & Grey-box Model & Rules & Yes & Yes & No \\
\hline$F D D$ & $\begin{array}{l}\text { Dexter and } \\
\text { Ngo }\end{array}$ & 1997 & HVAC & Grey-box Model & fuzzy logic & Yes & Yes & No \\
\hline$F D D$ & $\begin{array}{l}\text { Rossi and } \\
\text { Braun }\end{array}$ & 1997 & $A C$ & Statistical Model & Rules & Yes & Yes & No \\
\hline
\end{tabular}




\section{Appendix B R Code for Dynamic Bayesian Network Fault Diagnostics}

This section includes the $\mathrm{R}$ code for creating conditional probability table using leaky noise-max model, as well as a class object called DDBN (diagnostics Dynamic Bayesian Network) for creating DBN for fault diagnostics.

(implementations of the DDBN class for AHU and zone level diagnostics is available upon request due to length)

\# By default the first term in the probability list is always normal state

\# leak term is the default probability of the noise-max node when all its parents are normal

\# normal probability is always the constraint

child. 1 eak $=\mathrm{c}(0.9,0.1)$

\# prior probabilities for parents

parent1 $=\mathrm{c}(0.8,0.1,0.1)$

\# conditional probabilities, note that the normal condition $\mathrm{CP}$ is always the constraint $\mathrm{CP}$

\# which means for the normal $\mid$ normal CP it is always $p($ children=normal $\mid$ parent=normal) $=1$ child. parent $1=\mathrm{c}(0.4,0.6,0.1,0.9)$

addConstraints $=$ function $($ child_leak, $\operatorname{cpd})\{$

child_len $=$ length (child_leak)

constraints $=$ numeric (child_len)

constraints $[1]=1$

return (c (constraints, cpd)) \}

\#\# If a has levels a1, a2 and likewise for $\mathrm{b}$ and $\mathrm{c}$ then the order of values corresponds to the configurations

\#\# (a1, b1, c1), (a2, b1, c1) (a1, b2, c1), (a2, b2, c1) etc. That is, the first variable varies

\#\# fastest. Hence the first two elements in values will be the conditional probabilities

of a given

\#\# b=b1, c=c1

\#\# Some work functions

\# calculate cumulative pd from ppd

calculateCPD <- function (ppd) \{

$\mathrm{cpd}=\operatorname{cumsum}(\mathrm{ppd})$

$\mathrm{cpd}=\mathrm{cpd} /$ tail $(\mathrm{cpd}, \mathrm{n}=1)$

return (cpd)

\}

\# calculate ppd from cumulative pd 


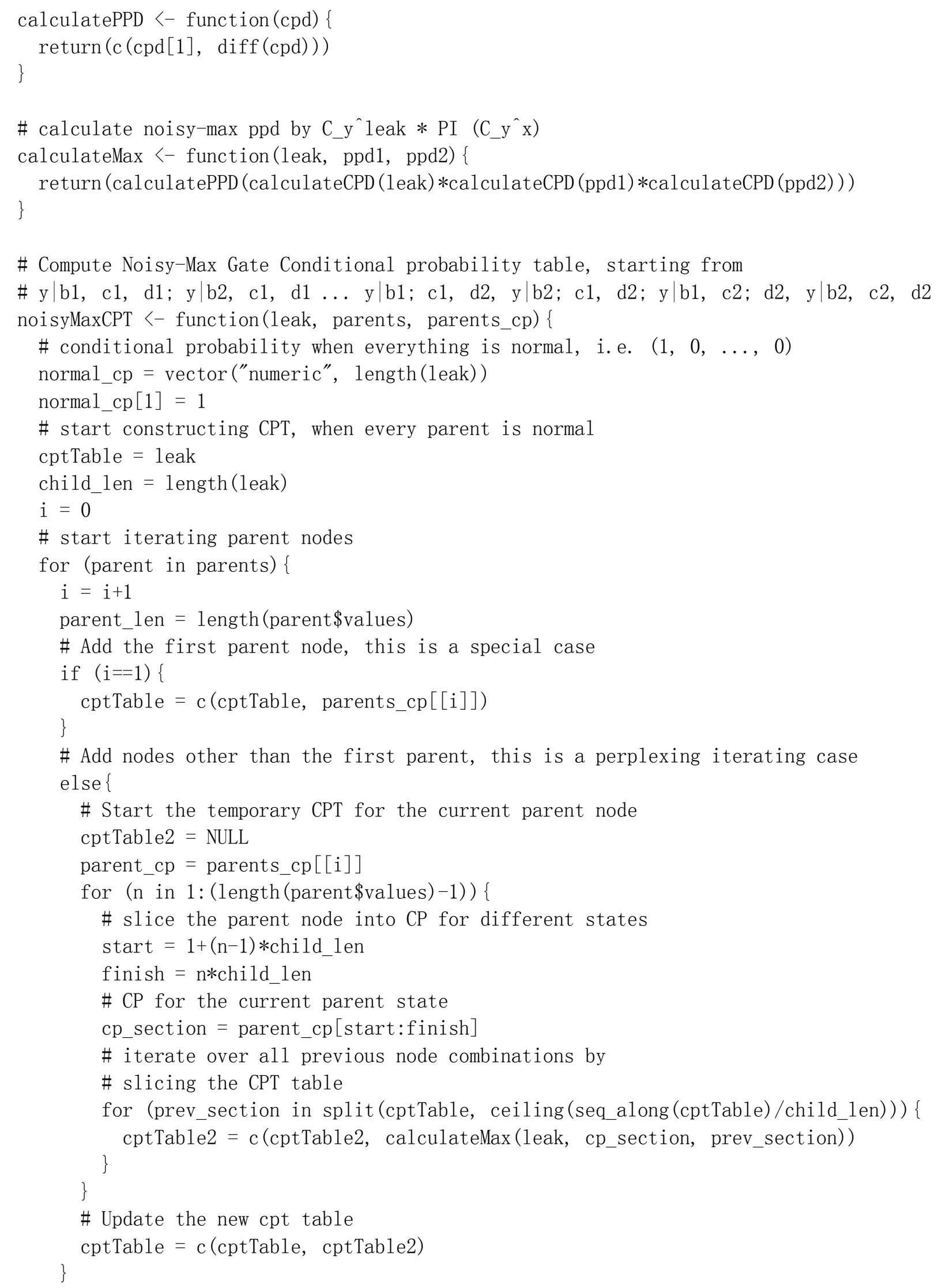


print (cptTable)

\} 


\section{Appendix C Python Code for Constrained Dual EKF}

This appendix shows the Python code the author developed for constrained dual EKF. For unconstrained estimation, filterPy and Pyestimate provides more powerful alternatives. (implementations of the constrained dual EKF for zone-level parameter estimation with R-C model is available upon request due to length)

import sympy

import numpy as np

import scipy. optimize

import SymPy

This is an implementation of constrained Dual Extended Kalman Filter used for combined state and parameter estimation

A tolerance is defined so that if the residual is small, parameter won't be updated

This allows for faster computation as well as more streamlined EKF tweaking

Inequality constraints is optional, but recommended when sensor noise is significant, or state model does not provide good abstraction

This class also uses SymPy to define its model function, as well as its state vector and parameter vector

class DualExtendedKalmanFilter:

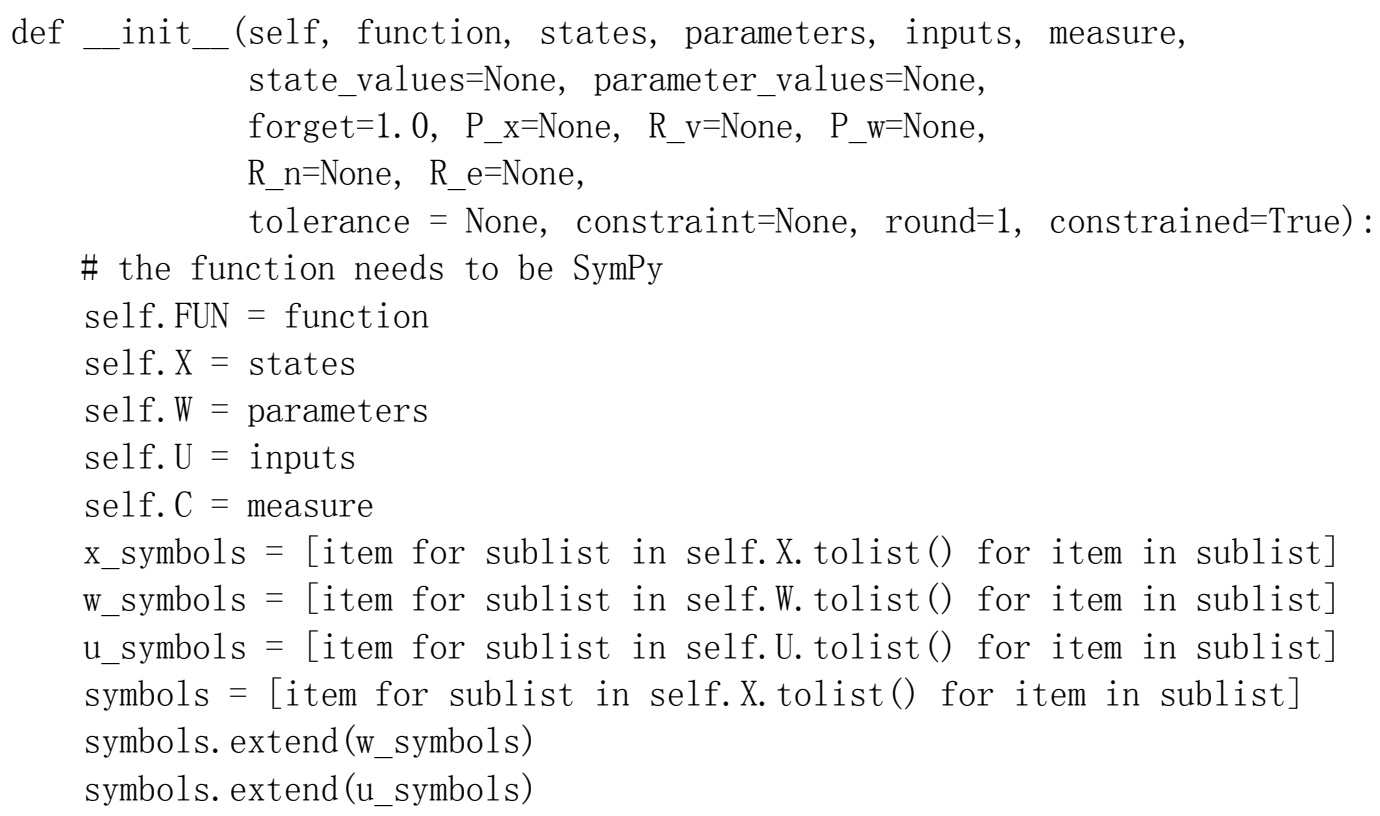




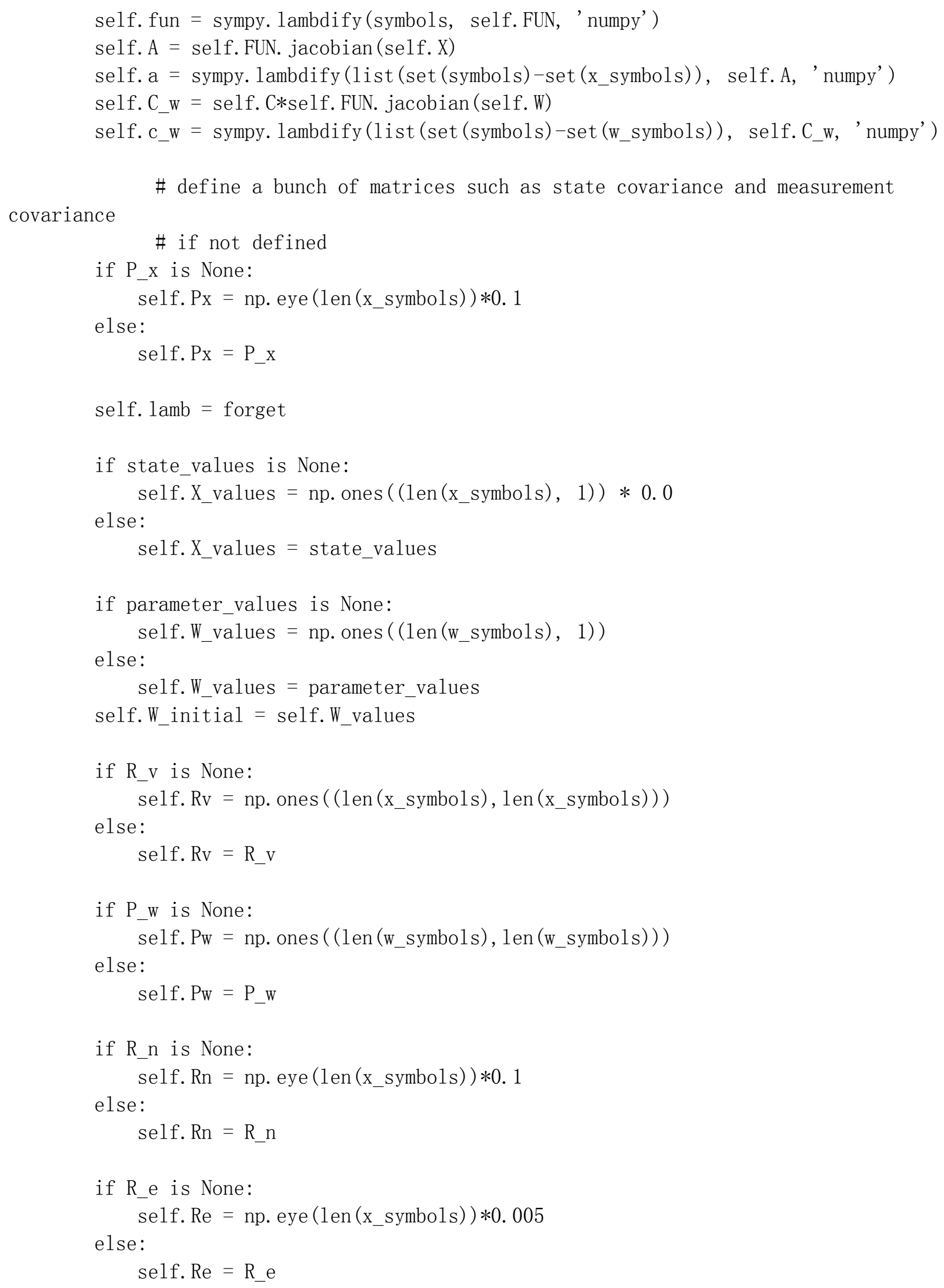




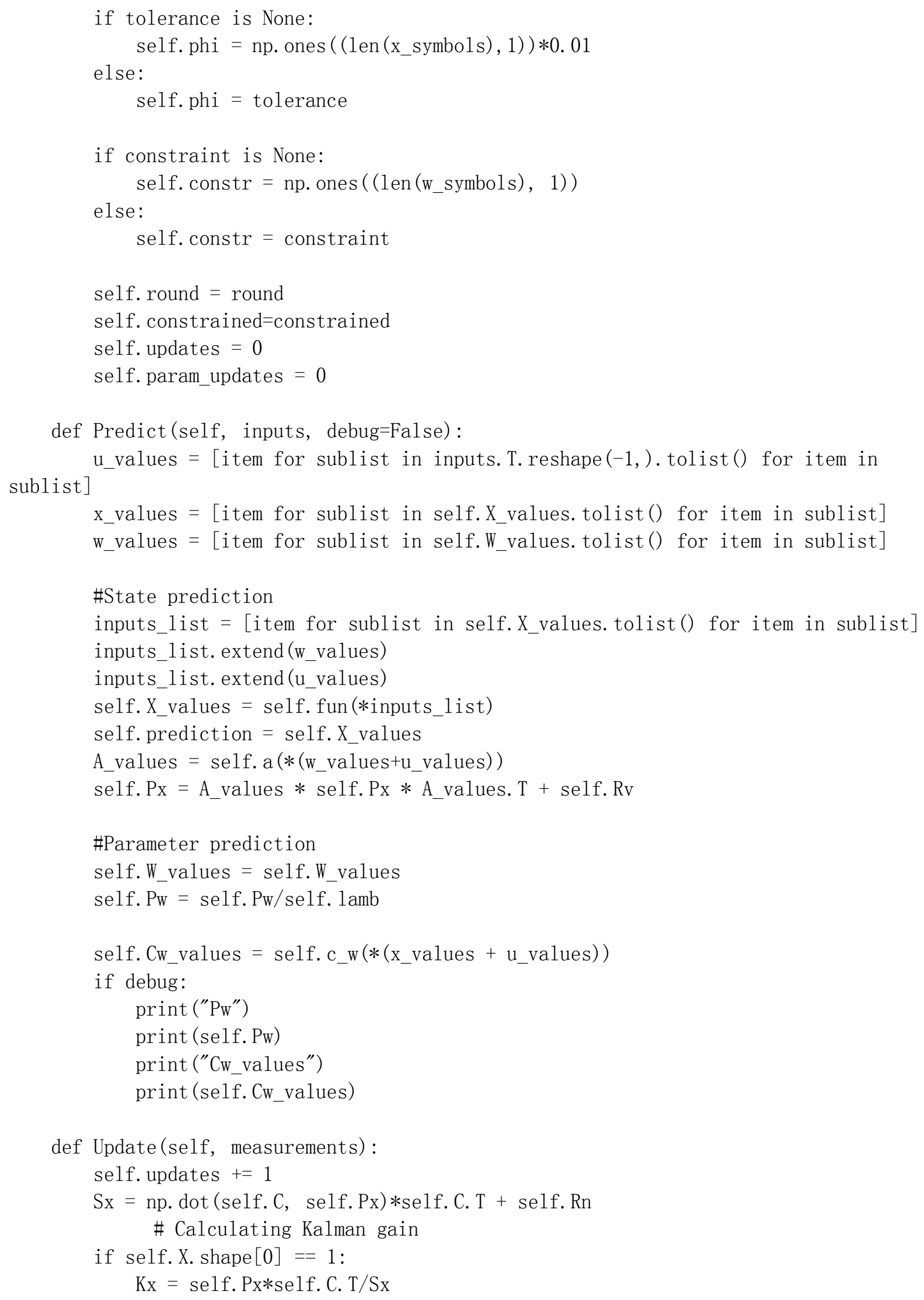

if tolerance is None: 
else:

$\mathrm{Kx}=$ self. Px*self. C. T*np. linalg. inv $(\mathrm{Sx})$

\# Updating values

self. $z=$ np. round (measurements, self. round) - np. round (self.C*self.X_values, self. round)

self.X_values_old $=$ self.X_values

self. $X \_v a l u e s=$ self. $X \_v a l u e s+K x *$ self. $z$

self. Px = (np. eye (self. Px. shape[0]) - Kx*self. C)*self. Px

if np. less (np. absolute(self. z), self.phi). all():

return

self. param_updates $+=1$

$\mathrm{Sw}=$ np. $\operatorname{dot}($ self. Cw_values, self. Pw $) *$ self. Cw_values. T + self. Re

if self. W. shape[0] $==1$ :

$\mathrm{Kw}=$ self. Pw*self. Cw_values. $\mathrm{T} / \mathrm{Sw}$

else:

try:

$\mathrm{Kw}=$ np. $\operatorname{dot}\left(\right.$ self. Pw, self. $\mathrm{Cw}_{-}$values. T) *np. linalg. inv $(\mathrm{Sw})$

except:

raise

\# singular matrix, most likely

self. $z 2=$ np. matrix $([[\operatorname{self} . z[0][0]],[0],[0],[0],[0]])$

self.W_values $=$ self.W_values + np. $\operatorname{dot}(\mathrm{Kw}$, self. $z 2)$

self. Pw $=$ (np. eye (self. Pw. shape[0]) - Kw*self. Cw_values) $*$ self. Pw

if (self. constrained is False):

\# False means no optimization is performed

return False

if (self.W_values $\langle 0)$. any () or (self.W_values $>$ self. constr). any () : res = scipy. optimize.fmin_cobyla(self.targetMin, self.W_initial,

[self. constrLow, self. constrHigh])

self. W_values $=$ res

\# True means unequality constraints are violated,

\# and an optimization is performed

\# note COBYLA used here only provides local minimum

\# COBYLA stands for: constrained optimization by linear

approximation

return True

else:

return False

def targetMin(self, $\mathrm{x})$ :

result $=\left(\mathrm{x}^{-}\right.$self. $\mathrm{W} \_$values $) . T *$ np. 1 inalg. inv (self. Pw $) *\left(\mathrm{x}^{-}\right.$self. W_values $)$

return result $[0,0]$

def constrLow (self, x): 
return $\mathrm{x}$

def constrHigh(self, x) :

return self. constr - $\mathrm{x}$ 


\section{Appendix D Python Code for Model-Cluster-Reduce}

Available as a library on Github: https://github.com/ZixiaoShi/epymcr 


\section{Bibliography}

[1] T. Vlachogianni and A. Valavanidis, "Energy and Environmental Impact on the Biosphere Energy Flow, Storage and Conversion in Human Civilization," Am. J. Educ. Res., vol. 1, no. 3 , pp. $68-78,2013$.

[2] U.S. Energy Information Administration, "International Energy Outlook 2017,” 2017.

[3] United Nations, "Paris Agreement," 2015.

[4] United Nations Environment Programme, "Buildings And Climate Change -- Summary for Decision-Makers," New York, USA, 2009.

[5] United States Green Building Council, “LEED | USGBC.” [Online]. Available: https://new.usgbc.org/leed. [Accessed: 21-Nov-2017].

[6] Passive House Institute, "Passivhaus.” [Online]. Available: http://www.passivehouse.com/02 informations/01_whatisapassivehouse/01_whatisapassi vehouse.htm. [Accessed: 21-Nov-2017].

[7] Q. Wu, V. Clulow, and S. Maslyuk, "Energy consumption inequality and human development," in Energy Efficiency - A Bridge to Low Carbon Economy, 2012, pp. 101116.

[8] UN Habitat, "11.1 Adequate Housing." [Online]. Available: https://unhabitat.org/unhabitat-for-the-sustainable-development-goals/11-1-adequate-housing/. [Accessed: 24Apr-2018]

[9] World Health Organization, "Housing and Health," WHO, 2011. [Online]. Available: http://www.who.int/hia/housing/en/. [Accessed: 24-Apr-2018].

[10] C. Turner and M. Frankel, "Energy Performance of LEED ${ }^{\circledR}$ for New Construction Buildings,” 2008.

[11] K. W. Roth, D. Westphalen, M. Y. Deng, P. Llana, and L. Quartararo, "Energy Impact of Commercial Building Controls and Performance Diagnostics : Market Characterization, 
Energy Impact of Building Faults and Energy Savings Potential," Cambridge, MA USA, 2005.

[12] I. L. Freeman and Building Research Establishment, "Building failure patterns and their implications," Garston, 1975.

[13] A. Behfar, D. Yuill, and Y. Yu, "Automated fault detection and diagnosis methods for supermarket equipment (RP-1615)," Sci. Technol. Built Environ., vol. 23, no. 8, pp. 1253$1266,2017$.

[14] S. Katipamula and M. Brambley, "Review Article: Methods for Fault Detection, Diagnostics, and Prognostics for Building Systems-A Review, Part I," HVAC\&R Res., vol. 11, no. 1, pp. 169-187, Apr. 2005.

[15] J. Belfast, "Fault diagnostics tools for commercial buildings - Applications, algorithms and barriers," Energy Eng., vol. 111, no. 3, pp. 57-78, 2014.

[16] M. Liu, "Improving Building Energy System Performance by Continuous Commissioning," Energy Eng., vol. 96, no. 5, pp. 46-56, 1999.

[17] S. Katipamula and M. Brambley, "Review Article: Methods for Fault Detection, Diagnostics, and Prognostics for Building Systems-A Review, Part II," HVAC\&R Res., vol. 11, no. 2, pp. 169-187, Apr. 2005.

[18] Federal Emergency Management Agency, "Introduction to Building code." 2017.

[19] "Early Detection of Building Faults Saves Future Expense," The Washington Post, p. $1952,1952$.

[20] S. X. Ding, Model-based fault diagnosis techniques: Design schemes, algorithms, and tools. Berlin, Heidelberg: Springer, 2008.

[21] R. Shoureshi and K. McLaughlin, "Microprocessor-Based Failure Detection of Heat Pumps," IFAC Proc. Vol., vol. 18, no. 9, pp. 155-160, 1985.

[22] P. B. Usoro, I. C. Schick, and S. Negahdaripour, "HVAC System Fault Detection and 
Diagnosis," in American Control Conference, 1985, pp. 606-612.

[23] L. K. Norford, J. A. Wright, R. A. Buswell, D. Luo, C. J. Klaassen, and A. Suby, "Demonstration of Fault Detection and Diagnosis Methods for Air-Handling Units (ASHRAE 1020-RP)," HVAC\&R Res., vol. 8, no. 1, 2002.

[24] A. Behfar, D. Yuill, and Y. Yu, "Automated fault detection and diagnosis methods for supermarket equipment (RP-1615),” Sci. Technol. Built Environ., vol. 23, no. 8, pp. 1253$1266,2017$.

[25] K. Bruton, P. Raftery, B. Kennedy, M. M. Keane, and D. T. J. O’Sullivan, "Review of automated fault detection and diagnostic tools in air handling units," Energy Effic., vol. 7, no. 2, pp. 335-351, 2014.

[26] S. Ginestet, D. Marchio, and O. Morisot, "Evaluation of faults impacts on energy consumption and indoor air quality on an air handling unit," Energy Build., vol. 40, no. 1, pp. 51-57, 2008.

[27] S. H. Lee and F. W. H. Yik, "A study on the energy penalty of various air-side system faults in buildings," Energy Build., vol. 42, no. 1, pp. 2-10, 2010.

[28] R. Isermann, Fault-diagnosis systems: An introduction from fault detection to fault tolerance. Berlin, Heidelberg: Springer, 2006.

[29] J. Rasmussen, "Diagnostic Reasoning in Action," IEEE Trans. Syst. Man Cybern., vol. 23, no. 4, pp. 981-992, 1993.

[30] P. Struss, a Malik, and M. Sachenbacher, "Qualitative Modeling Is the Key To Automated Diagnosis," Source, no. August, 2016.

[31] R. Isermann, Fault-Diagnosis Applications: Model-Based Condition Monitoring: Actuators, Drives, Machinery, Plants, Sensors, and Fault-tolerant Systems. 2011.

[32] V. Venkatasubramanian, “A review of process fault detection and diagnosis: Part III: Process history based methods," Comput. Chem. ..., vol. 27, pp. 293-311, 2003.

[33] D. M. Himmelblau, Fault Detection ond Diagnosis in Chemical and Petrochemicol 
Processes. American Institute of Chemical Engineers, 1978.

[34] V. Venkatasubramanian and R. Rengaswamy, "A review of process fault detection and diagnosis Part I : Quantitative model-based methods," Comput. Chem. ..., vol. 27, pp. 293-311, 2003.

[35] R. Isermann, "Model Based Fault Detection And Diagnosis Methods," in Proceedings of the American Control Conference, 1995, pp. 1605-1609.

[36] J. De Kleer and B. C. Williams, "Diagnosing multiple faults," Artif. Intell., vol. 32, no. 1987, pp. 97-130, 1987.

[37] V. Venkatasubramanian, R. Rengaswamy, and S. N. Kavuri, “A review of process fault detection and diagnosis: Part II: Qualitative models and search strategies," Comput. Chem. Eng., vol. 27, no. 3, pp. 313-326, 2003.

[38] W. Kim and S. Katipamula, "A review of fault detection and diagnostics methods for building systems," Sci. Technol. Built Environ., no. 0, pp. 1-19, 2017.

[39] Y. Yu, D. Woradechjumroen, and D. Yu, "A review of fault detection and diagnosis methodologies on air-handling units," Energy Build., vol. 82, pp. 550-562, 2014.

[40] R. Isermann, "Fault diagnosis of machines via parameter estimation and knowledge processing-Tutorial paper," Automatica, vol. 29, no. 4, pp. 815-835, 1993.

[41] T. Rossi and J. Braun, "A Statistical, Rule-Based Fault Detection and Diagnostic Method for Vapor Compression Air Conditioners," HVAC\&R Res., vol. 3, no. 1, pp. 19-37, 1997.

[42] X. Zhang, M. M. Polycarpou, and T. Parisini, "A robust detection and isolation scheme for abrupt and incipient faults in nonlinear systems," IEEE Trans. Automat. Contr., vol. 47, no. 4, pp. 576-593, 2002.

[43] S. A. McInerny and Y. Dai, "Basic vibration signal processing for bearing fault detection," IEEE Trans. Educ., vol. 46, no. 1, pp. 149-156, 2003.

[44] Z. Du, X. Jin, and Y. Yang, "Fault diagnosis for temperature, flow rate and pressure sensors in VAV systems using wavelet neural network," Appl. Energy, vol. 86, no. 9, pp. 
1624-1631, 2009.

[45] J. Wen and T. F. Smith, "Development and validation of online models with parameter estimation for a building zone with VAV system,” Energy Build., vol. 39, no. 1, pp. 1322, 2007.

[46] B. Kuipers, “Qualitative Simulation,” Artif. Intell., vol. 29, no. 1986, pp. 289-338, 1986.

[47] K. D. Forbus, “Qualitative Process Theory,” Massachusetts Institute of Technology, Boston, MA, 1984.

[48] C. Yu and C. Lee, "Fault Diagnosis Based on Qualitative/Quantitave Process Knowledge,” vol. 37, no. 4, pp. 617-628, 1991.

[49] L. Fornera, a. S. Glass, P. Gruber, and J. Tödtli, "Qualitative fault detection based on logical programming applied to a variable air volume air-handling unit," Control Eng. Pract., vol. 4, no. 1, pp. 105-116, 1996.

[50] J. Schein, S. T. Bushby, N. S. Castro, and J. M. House, "A rule-based fault detection method for air handling units (APAR)," Energy Build., vol. 38, no. 12, pp. 1485-1492, Dec. 2006.

[51] K. Bruton, D. Coakley, and P. Raftery, "Comparative analysis of the AHU InFO fault detection and diagnostic expert tool for AHUs with APAR," Energy Effic., no. September 2015, pp. 1-24, 2014.

[52] S. Bendapudi and J. E. Braun, "A review of literature on dynamic models of vapor compression equipment.," 2002.

[53] J. Lebrun, J.-P. Bourdouxhe, and M. Grodent, "HVAC toolkit: Algorithms and subroutines for primary HVAC system energy calculations," 1996.

[54] P. Haves, T. I. Salsbury, and J. A. Wright, "Condition monitoring in HVAC subsystems using first principles models," ASHRAE Trans., vol. 102, no. 1, pp. 519-527, 1996.

[55] Z. O’Neill, M. Shashanka, X. Pang, P. Bhattacharya, T. Bailey, and P. Haves, "Real Time Model-Based Energy Diagnostics in Buildings,” Proc. Build. Simul. 2011 12th Conf. Int. 
Build. Perform. Simul. Assoc., pp. 474-481, 2011.

[56] Z. O’Neill, X. Pang, M. Shashanka, P. Haves, and T. Bailey, “Model-based real-time whole building energy performance monitoring and diagnostics," J. Build. Perform. Simul., vol. 7, no. 2, pp. 83-99, 2013.

[57] Y. Ham and M. Golparvar-Fard, "EPAR: Energy Performance Augmented Reality models for identification of building energy performance deviations between actual measurements and simulation results," Energy Build., vol. 63, pp. 15-28, 2013.

[58] J. M. Gordon and K. C. Ng, "Predictive and diagnostic aspects of a universal thermodynamic model for chillers," Int. J. Heat Mass Transf., vol. 38, no. 5, pp. 807-818, 1995.

[59] J. M. Gordon, K. C. Ng, H. T. Chua, and C. K. Lim, "How varying condenser coolant flow rate affects chiller performance: Thermodynamic modeling and experimental confirmation," Appl. Therm. Eng., vol. 20, no. 13, pp. 1149-1159, 2000.

[60] Y. Jia and T. A. Reddy, "Characteristic physical parameter approach to modeling chillers suitable for fault detection, diagnosis, and evaluation," J. Sol. energy Eng., vol. 125, no. 3, pp. 258-265, 2003.

[61] J. E. Braun, "Reducing Energy Costs and Peak Electrical Demand through Optimal Control of Building Thermal Storage," J. Chem. Inf. Model., vol. 53, p. 160, 1989.

[62] J. Braun and N. Chaturvedi, “An Inverse Gray-Box Model for Transient Building Load Prediction," HVAC\&R Res., vol. 8, no. 1, pp. 73-99, 2002.

[63] P. Radecki and B. Hencey, “Online Building Thermal Parameter Estimation via Unscented Kalman Filtering,” Am. Control Conf. (ACC), 2012, pp. 3056-3062, 2012.

[64] B. Sun, P. B. Luh, Q. S. Jia, Z. O’Neill, and F. Song, "Building energy doctors: An SPC and Kalman Filter-based method for system-level fault detection in HVAC systems," IEEE Trans. Autom. Sci. Eng., vol. 11, no. 1, pp. 215-229, 2014.

[65] G. P. Henze, C. Felsmann, and G. Knabe, "Evaluation of optimal control for active and 
passive building thermal storage," Int. J. Therm. Sci., vol. 43, no. 2, pp. 173-183, 2004.

[66] B. James, "Load Control Using Building Thermal Mass," Trans. ASME, vol. 125, no. August, p. 292, 2003.

[67] Y. Ma, A. Kelman, A. Daly, and F. Borrelli, "Predictive Control for Energy Efficient Buildings with Thermal Storage: Modeling, Stimulation, and Experiments," IEEE Control Syst., vol. 32, no. 1, pp. 44-64, 2012.

[68] H. B. Gunay, J. Bursill, B. Huchuk, W. O’Brien, and I. Beausoleil-Morrison, “Shortestprediction-horizon model-based predictive control for individual offices," Build. Environ., vol. 82, pp. 408-419, 2014.

[69] Z. Shi, W. O’Brien, and B. Gunay, "Building Zone Fault Detection with Kalmnan Filter based Methods," in eSim 2016, 2016.

[70] Y. Gao, S. Liu, F. Li, and Z. Liu, "Fault detection and diagnosis method for cooling dehumidifier based on LS-SVM NARX model," Int. J. Refrig., vol. 61, pp. 69-81, 2016.

[71] Z. Du and X. Jin, "Detection and diagnosis for sensor fault in HVAC systems," Energy Convers. Manag., vol. 48, no. 3, pp. 693-702, 2007.

[72] N. A. Sulaiman, M. F. Othman, and H. Abdullah, "Fuzzy Logic Control and Fault Detection in Centralized Chilled Water System," in IEEE Symposium Series on Computational Intelligence, 2015, pp. 8-13.

[73] C. H. Lo, P. T. Chan, Y. K. Wong, a. B. Rad, and K. L. Cheung, "Fuzzy-genetic algorithm for automatic fault detection in HVAC systems," Appl. Soft Comput. J., vol. 7, no. 2, pp. 554-560, 2007.

[74] X. Li, M. Shao, and L. Ding, "HVAC Fault Diagnosis System Using Rough Set Theory and Support Vector Machine,” 2009 Second Int. Work. Knowl. Discov. Data Min., no. 2007, pp. 895-899, 2009.

[75] H. Wang, Y. Chen, C. W. H. Chan, and J. Qin, "An online fault diagnosis tool of VAV terminals for building management and control systems," Autom. Constr., vol. 22, pp. 
203-211, 2012.

[76] Y. Zhao, F. Xiao, and S. Wang, “An intelligent chiller fault detection and diagnosis methodology using Bayesian belief network,” Energy Build., vol. 57, pp. 278-288, 2013.

[77] S. Li and J. Wen, “A model-based fault detection and diagnostic methodology based on PCA method and wavelet transform," Energy Build., vol. 68, no. PARTA, pp. 63-71, 2014.

[78] C. B. Jones, "Fault detection and diagnostics of an HVAC sub-system using adaptive resonance theory neural networks," University of New Mexico, 2015.

[79] L. Burgas, J. Melendez, and J. Colomer, "Principal component analysis for monitoring electrical consumption of academic buildings," Energy Procedia, vol. 62, pp. 555-564, 2014.

[80] F. Marino et al., "Indoor lighting fault detection and diagnosis using a data fusion approach,” WIT Trans. Ecol. Environ., vol. 190 VOLUME, no. APRIL, pp. 83-94, 2014.

[81] G. Lin and D. E. Claridge, "A temperature-based approach to detect abnormal building energy consumption,” Energy Build., vol. 93, pp. 110-118, 2015.

[82] Z. O’Neill and C. O’Neill, "Development of a probabilistic graphical model for predicting building energy performance," Appl. Energy, vol. 164, pp. 650-658, 2016.

[83] R. Martinez-Guerra and J. L. Mata-Machuca, Fault Detection and Diagnosis in Nonlinear Systems: A Differential and Algebraic Viewpoint. Springer International Publishing Switzerland, 2014.

[84] W.-Y. Lee, C. Park, and G. E. Kelly, "Fault Detection in an Air-Handling Unit Using Residual and Recursive Parameter Identification Methods," ASHRAE Transactions, vol. 102, no. 1. pp. 1-12, 1996.

[85] S. A. Kalogirou, “Applications of artificial neural-networks for energy systems," Appl. Energy, vol. 67, pp. 17-35, 2000.

[86] D. Dehestani, S. Su, H. Nguyen, and Y. Guo, "Robust fault tolerant application for HVAC 
system based on combination of online SVM and ANN black box model," Control Conf. (ECC), 2013 Eur., pp. 2976-2981, 2013.

[87] S. Wang and F. Xiao, "AHU sensor fault diagnosis using principal component analysis method," Energy Build., vol. 36, no. 2, pp. 147-160, Feb. 2004.

[88] S. Wang and J. Cui, "Sensor-fault detection, diagnosis and estimation for centrifugal chiller systems using principal-component analysis method," Appl. Energy, vol. 82, no. 3, pp. 197-213, Nov. 2005.

[89] Z. Du, B. Fan, J. Chi, and X. Jin, "Sensor fault detection and its efficiency analysis in air handling unit using the combined neural networks," Energy Build., vol. 72, pp. 157-166, 2014.

[90] K. A. Cort and H. Cho, "Final Project Report: Self-Correcting Controls for VAV System Faults Filter / Fan / Coil and VAV Box Sections," 2011.

[91] F. Lauro et al., "Building fan coil electric consumption analysis with fuzzy approaches for fault detection and diagnosis," Energy Procedia, vol. 62, pp. 411-420, 2014.

[92] D. Wijayasekara, O. Linda, M. Manic, and C. Rieger, "Mining Building Energy Management System Data Using Fuzzy Anomaly Detection and Linguistic Descriptions,” IEEE Trans. Ind. INFORMATICS, vol. 10, no. 3, pp. 1829-1840, 2014.

[93] B. Fan, Z. Du, X. Jin, X. Yang, and Y. Guo, “A hybrid FDD strategy for local system of AHU based on artificial neural network and wavelet analysis," Build. Environ., vol. 45, no. 12 , pp. $2698-2708,2010$.

[94] Q. Zhou, S. Wang, and F. Xiao, "A novel strategy for the fault detection and diagnosis of centrifugal chiller systems," HVAC R Res., vol. 15, no. 1, pp. 57-75, 2009.

[95] Z. Hou, Z. Lian, Y. Yao, and X. Yuan, "Data mining based sensor fault diagnosis and validation for building air conditioning system," Energy Convers. Manag., vol. 47, pp. 2479-2490, 2006.

[96] Z. Du, B. Fan, X. Jin, and J. Chi, "Fault detection and diagnosis for buildings and HVAC 
systems using combined neural networks and subtractive clustering analysis," Build. Environ., vol. 73, pp. 1-11, 2013.

[97] B. Cai et al., "Multi-source information fusion based fault diagnosis of ground-source heat pump using Bayesian network," Appl. Energy, vol. 114, pp. 1-9, 2014.

[98] Y. Zhao, J. Wen, and S. Wang, "Diagnostic Bayesian networks for diagnosing air handling units faults - Part I: Faults in dampers, fans, filters and sensors," Appl. Therm. Eng., vol. 111, pp. 1272-1286, 2017.

[99] Y. Zhao, J. Wen, and S. Wang, "Diagnostic Bayesian networks for diagnosing air handling units faults - Part II: Faults in coils and sensors," Appl. Therm. Eng., vol. 90, no. October, pp. 145-157, 2015.

[100] K. Yan, W. Shen, T. Mulumba, and A. Afshari, "ARX model based fault detection and diagnosis for chillers using support vector machines," Energy Build., vol. 81, pp. 287-295, 2014.

[101] J. Liang and R. Du, "Model-based Fault Detection and Diagnosis of HVAC systems using Support Vector Machine method," Int. J. Refrig., vol. 30, no. 6, pp. 1104-1114, Sep. 2007.

[102] D. Nikovski, "Constructing Bayesian networks for medical diagnosis from $\backslash$ nincomplete and partially correct statistics," IEEE Trans. Knowl. Data Eng., vol. 12, no. 4, pp. 1-18, 2000.

[103] W. Kim and S. Katipamula, "A review of fault detection and diagnostics methods for building systems," Sci. Technol. Built Environ., no. 0, pp. 1-19, 2017.

[104] Z. O’Neill, X. Pang, M. Shashanka, P. Haves, and T. Bailey, "Model-based real-time whole building energy performance monitoring and diagnostics," J. Build. Perform. Simul., vol. 7, no. 2, pp. 83-99, 2014.

[105] H. Cheung and J. E. Braun, "Development of Fault Models for Hybrid Fault Detection and Diagnostics Algorithm Development of Fault Models for Hybrid Fault Detection and 
Diagnostics Algorithm," 2015.

[106] R. Zhang and T. Hong, "Modeling of HVAC operational faults in building performance simulation," Appl. Energy, vol. 202, pp. 178-188, 2017.

[107] ASHRAE, ASHRAE Guideline 14-2014: Measurement of Energy, Demand, and Water Savings. 2014.

[108] K. Bruton, P. Raftery, P. O’Donovan, N. Aughney, M. M. Keane, and D. T. J. O’Sullivan, "Development and alpha testing of a cloud based automated fault detection and diagnosis tool for Air Handling Units," Autom. Constr., vol. 39, pp. 70-83, 2014.

[109] D. B. Araya, K. Grolinger, H. F. Elyamany, M. A. M. Capretz, and G. Bitsuamlak, "Collective Contextual Anomaly Detection Framework for Smart Buildings," in Neural Networks (IJCNN), 2016 International Joint Conference on, 2016, pp. 24-29.

[110] B. Dong, Z. O’Neill, and Z. Li, “A BIM-enabled information infrastructure for building energy Fault Detection and Diagnostics," Autom. Constr., vol. 44, pp. 197-211, Aug. 2014.

[111] G. Provan, J. Ploennigs, M. Boubekeur, A. E. Mady, and A. Ahmed, "Using BIM data for generating and updating diagnostic models,” ... Conf. Civil, ..., no. 1, pp. 1-2, 2009.

[112] D. J. Spiegelhalter and R. P. Knill-Jones, "Statistical and Knowledge-Based Approaches to Clinical Decision-Support Systems, with an Application in Gastroenterology Author ( $\mathrm{s}$ ): David J . Spiegelhalter and Robin P . Knill-Jones Source : Journal of the Royal Statistical Society . Series A ( Genera," J. R. Stat. Soc., vol. 147, no. 1, pp. 35-77, 1984.

[113] P. Szolovits and S. G. Pauker, "Categorical and probabilistic reasoning in medical diagnosis," Artif. Intell., vol. 11, no. 1-2, pp. 115-144, 1978.

[114] D. Dey and B. Dong, "A probabilistic approach to diagnose faults of air handling units in buildings," Energy Build., vol. 130, pp. 177-187, 2016.

[115] V. Machairas, A. Tsangrassoulis, and K. Axarli, “Algorithms for optimization of building 
design: A review,” Renew. Sustain. Energy Rev., vol. 31, no. 1364, pp. 101-112, 2014.

[116] P. M. Van Every, M. Rodriguez, C. Birk Jones, A. A. Mammoli, and M. Martínez-Ramón, "Advanced detection of HVAC faults using unsupervised SVM novelty detection and Gaussian process models," Energy Build., vol. 149, pp. 216-224, 2017.

[117] F. J. Diez and M. J. Druzdzel, “Canonical Probabilistic Models for Knowledge Engineering," Madrid, Spain, 2000.

[118] D. Koller and N. Friedman, Probabilistic Graphical Models: Principles and Techniques. 2010.

[119] M. W. Vanik, J. L. Beck, and S. K. Au, "Bayesian Probabilistic Appraoch to Structural Health Monitoring,” J. Eng. Mech., vol. 126, no. July, pp. 738-745, 2000.

[120] R. Sharifi and R. Langari, "Nonlinear sensor fault diagnosis using mixture of probabilistic PCA models,” Mech. Syst. Signal Process., vol. 85, pp. 638-650, 2017.

[121] H. Boudali and J. B. Dugan, "A discrete-time Bayesian network reliability modeling and analysis framework," Reliab. Eng. Syst. Saf., vol. 87, no. 3, pp. 337-349, 2005.

[122] M. J. Daigle, “A Qualitative Event Based Approach to Fault Diagnosis of Hybrid Systems," Vanderbilt University, 2008.

[123] S. Wang and X. Xu, "Simplified building model for transient thermal performance estimation using GA-based parameter identification," Int. J. Therm. Sci., vol. 45, no. 4, pp. 419-432, 2006.

[124] M. Maasoumy, B. Moridian, M. Razmara, M. Shahbakhti, and A. SangiovanniVincentelli, “Online Simultaneous State Estimation and Parameter Adaptation for Building Predictive Control," in ASME 2013 Dynamic Systems and Control Conference, 2013.

[125] A. H. Mohsenian-Rad and A. Leon-Garcia, "Optimal residential load control with price prediction in real-time electricity pricing environments," IEEE Trans. Smart Grid, vol. 1, 
no. 2, pp. 120-133, 2010.

[126] T. A. Wenzel, K. J. Burnham, M. V. Blundell, and R. A. Williams, "Dual extended Kalman filter for vehicle state and parameter estimation," Veh. Syst. Dyn. Int. J. Veh. Mech. Mobil., vol. 44, no. 2, pp. 153-171, 2006.

[127] G. Chowdhary and R. Jategaonkar, "Aerodynamic parameter estimation from flight data applying extended and unscented Kalman filter," Aerosp. Sci. Technol., vol. 14, no. 2, pp. 106-117, 2010.

[128] M. C. Vandyke, J. L. Schwartz, and C. D. Hall, “Unscented Kalman Filtering for Spacecraft Attitude State and Parameter Estimation," Proc. AAS/AIAA Sp. Flight Mech. Conf. no. AAS 04-115, (Maui, pp. 1-13, 2004.

[129] S. Haykin, Kalman filtering and neural networks. New York, USA: Wiley, 2001.

[130] Y. Yan, P. B. Luh, and B. Sun, "Fault detection of cooling coils based on unscented Kalman filters and statistical process control,” IEEE Int. Conf. Autom. Sci. Eng., pp. 237$242,2013$.

[131] C.-C. Lin and L. (Leon) Wang, "Forecasting simulations of indoor environment using data assimilation via an Ensemble Kalman Filter,” Build. Environ., vol. 64, pp. 169-176, 2013.

[132] G. Platt, J. Li, R. Li, G. Poulton, G. James, and J. Wall, “Adaptive HVAC zone modeling for sustainable buildings," Energy Build., vol. 42, no. 4, pp. 412-421, 2010.

[133] A. T. Nelson, "Nonlinear estimation and modeling of noisy time series by dual Kalman filtering methods," 2000.

[134] E. a Wan and A. T. Nelson, "Dual extended kalman filter methods," in Kalman filtering and neural networks, New York, USA: John Wiley \& Sons, 2001, pp. 123-173.

[135] M. St-Pierre and D. Gingras, "Comparison between the unscented Kalman filter and the extended Kalman filter for the position estimation module of an integrated navigation information system," IEEE Intell. Veh. Symp. 2004, pp. 831-835, 2004.

[136] E. A. Wan and R. Van Der Merwe, “The unscented Kalman filter for nonlinear 
estimation," in Adaptive Systems for Signal Processing, Communications, and Control Symposium 2000. AS-SPCC. The IEEE 2000, 2000, pp. 153-158.

[137] E. a Wan and A. T. Nelson, "Dual Kalman Filtering Methods for Nonlinear Prediction, Smoothing and Estimation,” Adv. Neural Inf. Process. Syst. 9, no. 1, pp. 793-799, 1997.

[138] S. Ungarala, E. Dolence, and K. Li, "Constrained extended Kalman filter for nonlinear state estimation," 8th Int. IFAC Symp. Dyn. Control Process Syst., vol. 2, pp. 63-68, 2007.

[139] G. Lillacci and M. Khammash, "Parameter estimation and model selection in computational biology," PLoS Comput. Biol., vol. 6, no. 3, 2010.

[140] A. Meurer et al., "SymPy: symbolic computing in Python," PeerJ Comput. Sci., vol. 3, p. e103, 2017.

[141] M. J. D. Powell, “A view of algorithms for optimization without derivatives," Cambridge Univ. Tech. Rep. DAMTP, no. 3, 2007.

[142] E. Jones, T. Oliphant, P. Peterson, and others, “ $\{\mathrm{SciPy}\}$ : Open source scientific tools for \{Python\}." .

[143] R. R. J. Labbe, "FilterPy - Kalman filters and other optimal and non-optimal estimation filters in Python,” 2015. [Online]. Available: https://github.com/rlabbe/filterpy.

[144] Pykalman, "pykalman, Kalman Filter, Smoother, and EM Algorithm for Python." [Online]. Available: https://github.com/pykalman/pykalman.

[145] X.-B. Yang, X.-Q. Jin, Z.-M. Du, and Y.-H. Zhu, “A novel model-based fault detection method for temperature sensor using fractal correlation dimension," Build. Environ., vol. 46, no. 4, pp. 970-979, 2011.

[146] Z. Du, X. Jin, and X. Yang, “A robot fault diagnostic tool for flow rate sensors in air dampers and VAV terminals," Energy Build., vol. 41, pp. 279-286, 2008.

[147] F. Xiao, Y. Zhao, J. Wen, and S. Wang, "Bayesian network based FDD strategy for variable air volume terminals," Autom. Constr., vol. 41, pp. 106-118, 2014.

[148] D. Heckerman, "A Tutorial on Learning With Bayesian Networks," Innov. Bayesian 
Networks, vol. 1995, no. November, pp. 33-82, 1996.

[149] C. E. Kahn, L. M. Roberts, K. A. Shaffer, and P. Haddawy, "Construction of a Bayesian network for mammographic diagnosis of breast cancer," Comput. Biol. Med., vol. 27, no. 1, pp. 19-29, 1997.

[150] G. Arroyo-Figueroa and L. E. Sucar, "A temporal Bayesian network for diagnosis and prediction," Proc. 15th Annu. Conf. Uncertain. Artif. Intell., pp. 13-20, 1999.

[151] H. Kao, C. Huang, and H. Li, "Supply chain diagnostics with dynamic Bayesian networks," Comput. Ind. Eng., vol. 49, no. 2, pp. 339-347, 2005.

[152] Z. Yongli, H. Limin, and L. Jinling, "Bayesian networks-Based approach for power systems fault diagnosis," IEEE Trans. Power Deliv., vol. 21, no. 2, pp. 634-639, 2006.

[153] D. A. Tobon-Mejia, K. Medjaher, and N. Zerhouni, "CNC machine tool's wear diagnostic and prognostic by using dynamic Bayesian networks," Mech. Syst. Signal Process., vol. 28, pp. 167-182, 2012.

[154] Y. Zhao, J. Wen, F. Xiao, X. Yang, and S. Wang, "Diagnostic Bayesian networks for diagnosing air handling units faults - part I: Faults in dampers, fans, filters and sensors," Appl. Therm. Eng., vol. 111, pp. 1272-1286, 2017.

[155] Z. Wang, Z. Wang, S. He, X. Gu, and Z. F. Yan, "Fault detection and diagnosis of chillers using Bayesian network merged distance rejection and multi-source non-sensor information," Appl. Energy, vol. 188, pp. 200-214, 2017.

[156] D. Nikovski, “Constructing Bayesian Networks for Medical Diagnosis from Incomplete and Partially Correct Statistics," IEEE Trans. Knowl. Data Eng., vol. 12, no. 4, pp. 509$516,2000$.

[157] S. Dey and J. A. Stori, “A Bayesian network approach to root cause diagnosis of process variations," Int. J. Mach. Tools Manuf., vol. 45, no. 1, pp. 75-91, 2005.

[158] K. P. Murphy, "Dynamic Bayesian Networks: Representation, Inference and Learning,” 
2002.

[159] D. Margaritis, "Learning Bayesian Network Model Structure from Data," 2003.

[160] K. Murphy, “Dynamic Bayesian Networks,” vol. 2002, no. 11. pp. 1274-1288, 2002.

[161] D. A. Tobon-Mejia, K. Medjaher, and N. Zerhouni, “CNC machine tool's wear diagnostic and prognostic by using dynamic Bayesian networks," Mech. Syst. Signal Process., vol. 28, pp. 167-182, 2012.

[162] S. H. Højsgaard, "Graphical Independence Networks with the gRain Package for R,” J. Stat. Softw., vol. 46, no. 10, pp. 1-26, 2012.

[163] S. L. Lauritzen and D. J. Spiegelhalter, "Local Computations with Probabilities on Graphical Structures and Their Application to Expert Systems,” J. R. Stat. Soc., vol. 50, no. 2, pp. 157-224, 1988.

[164] G. F. Cooper, "The Computational Complexity of Probabilistic Inference Using Bayesian Belief Network," Artificial Intelligence, no. 42. pp. 393-405, 1990.

[165] F. V. Jensen, K. G. Olesen, and S. K. Andersen, “An algebra of bayesian belief universes for knowledge- based systems," Networks, vol. 20, no. 5, pp. 637-659, 1990.

[166] P. P. Shenoy and G. Shafer, "Propagating Belief Functions with Local Computations," IEEE Expert, vol. 1, no. 3, pp. 43-52, 1986.

[167] V. Lepar and P. Shenoy, “A comparison of Lauritzen-Spiegelhalter, Hugin, and ShenoyShafer architectures for computing marginals of probability distributions," Proc.

Fourteenth Conf. ..., vol. 14, no. x, pp. 328-337, 1998.

[168] Z. Shi and W. O’Brien, "Development of a distributed fault detection, diagnostic and evlauation framework," ASHRAE Trans., 2018.

[169] A. Zagorecki and M. J. Druzdzel, "Knowledge engineering for bayesian networks: How common are noisy-MAX distributions in practice'," IEEE Trans. Syst. Man, Cybern. Part ASystems Humans, vol. 43, no. 1, pp. 186-195, 2013.

[170] P. C. Kraaijeveld and M. J. Druzdzel, “GeNIeRate : An Interactive Generator of 
Diagnostic Bayesian Network Models," in 16th International Workshop on Principles of Diagnosis, 2005, pp. 175-180.

[171] C. Fan, F. Xiao, and C. Yan, "A framework for knowledge discovery in massive building automation data and its application in building diagnostics," Autom. Constr., vol. 50, no. C, pp. 81-90, 2015.

[172] B. Eisenhower, Z. O’Neill, S. Narayanan, V. A. Fonoberov, and I. Mezić, “A methodology for meta-model based optimization in building energy models," Energy Build., vol. 47, no. April, pp. 292-301, 2012.

[173] Mangesh Basarkar, X. Pang, L. Wang, P. Haves, and T. Hong, "Modeling and simulation of HVAC faults in EnergyPlus," IBPSA Build. Simul., pp. 14-16, 2013.

[174] X. Pang, M. Wetter, P. Bhattacharya, and P. Haves, “A framework for simulation-based real-time whole building performance assessment," Build. Environ., vol. 54, pp. 100-108, 2012.

[175] W. J. Cole, E. T. Hale, and T. F. Edgar, "Building energy model reduction for model predictive control using OpenStudio," in American Control Conference (ACC), 2013, pp. $449-454$.

[176] C. van Treeck and E. Rank, "Dimensional reduction of 3D building models using graph theory and its application in building energy simulation," Eng. Comput., vol. 23, no. 2, pp. 109-122, 2007.

[177] B. Eisenhower, T. Maile, M. Fischer, and I. Mezić, "Decomposing Building System Data for Model Validation and Analysis Using the Koopman Operator," in SimBuild 2010, 2010, pp. 434-441.

[178] Z. Shi and W. O’Brien, "Building energy model reduction using model-cluster-reduce pipeline," J. Build. Perform. Simul., 2017.

[179] B. J. Frey and D. Dueck, "Clustering by passing messages between data points," Science 
(80-. )., vol. 315, no. 5864, pp. 972-977, 2007.

[180] Z. Shi and W. O’Brien, "Building Energy Model Reduction using Principal Component Analysis and Affinity Propagation Clustering of Thermal Zones," in 3rd Asia Conference of International Building Performance Simulation Association, 2016.

[181] D. Crawley et al., "EnergyPlus: creating a new-generation building energy simulation program," Energy Build., vol. 33, no. 4, pp. 319-331, 2001.

[182] S. Bengea et al., "Parameter estimation of a building system model and impact of estimation error on closed-loop performance," in Proceedings of the IEEE Conference on Decision and Control, 2011, pp. 5137-5143.

[183] J. Wen and S. Li, “ASHRAE 1312-RP: Tools for Evaluating Fault Detection and Diagnostic Methods for Air-Handling Units,” Atlanta, GA, 2011.

[184] N. Fernandez, M. R. Brambley, S. Katipamula, H. Cho, J. Goddard, and L. Dinh, "SelfCorrecting HVAC Controls Project Final Report," Pacific Northwest Natl. Lab., no. December, 2009.

[185] Z. Shi and W. O’Brien, “Using Building Performance Simulation for Fault Impact Evaluation," in eSim 2018, 2018. 